\title{
Estimation of Dynamic Rate Parameters in Insect Populations Undergoing Sublethal Exposure to Pesticides
}

\author{
H. T. Banks*, John E. Banks ${ }^{\dagger}$, Lara K. Dick* ${ }^{*}$ John D. Stark ${ }^{\ddagger}$ \\ *Center for Research in Scientific Computation \\ North Carolina State University \\ Raleigh, North Carolina 27695-8205 \\ $\dagger$ Environmental Science \\ Interdisciplinary Arts and Sciences \\ University of Washington \\ Tacoma, Washington 98402-3100 \\ ${ }^{\ddagger}$ Department of Entomology \\ Ecotoxicology Program \\ Washington State University \\ Puyallup, Washington 98371
}

June 30, 2005

\begin{abstract}
With newer, more environmentally friendly and, subsequently less lethal, pesticides in use, evaluating efficacy of a pesticide now requires more than simply counting deaths after treatment. A deterministic, age-structured matrix model that incorporates a species' life history traits (such as birth rate, death rate and fecundity) has previously been used by ecologists. This model will be presented and discussed along with an alternative continuous, age-structured model which offers significant advantage in considering sublethal damage. We use this continuous model to estimate time-dependent mortality parameters in a least-squares technique. Confidence intervals are given and results from tests for statistical significance of added parameters are presented.
\end{abstract}

Key Words: Population models; Leslie; Sinko-Streifer; McKendrick-VonFoerster; time-dependent parameters

\section{Introduction}

Motivated by environmental concerns, entomologists are being forced to understand the effects of new classes of pesticides and insecticides that are less lethal than their predecessors. These new pesticides act as growth regulators, causing scientists to be concerned with survival and recovery 
delays exhibited by species targeted by the pesticide. Determining the efficacy of a pesticide requires information about life history traits. In this report, we will describe models that utilize these traits, such as birth rate, death rate and fecundity, to describe population growth.

We will study aphids, who reproduce on day five or six of their life span. Since a two day old aphid and a ten day old aphid will exhibit different life traits, they provide an excellent subject for age-dependent effects. Thus, in order to understand how a pesticide affects growth and reproduction, one must know something about the individual's age class. We first employ Leslie age-structured matrix projection models, and discuss simulations of population growth, compare different exposure methods, report various results, and mention previously documented conclusions. As we shall see, these matrix models, while widely accepted in the ecology community, are not adequate to treat sublethal damage that is prevalent in new classes of insecticides. We then turn to continuous age-structured models which to date are not widely used in the community. We should not expect these models, which are more conceptually challenging, to be embraced by the community. As Caswell [C] has noted, "It took 25 years for ecologists to adopt matrix population models. This was partly because matrix algebra was perceived as an advanced and esoteric branch of mathematics." But in our efforts below, we believe we make a compelling case for the adoption of these models by researchers.

\section{Discrete Age-Structured Models}

\subsection{Experimental Procedure and Data Description}

In 1995, Stark and Wennergren [SW] argued that demographic toxicological analysis estimating the total effect of insecticides on populations was best completed using a stage-structured approach. They argued that demographic toxicology is an ecotoxicological technique that incorporates life table parameters in the context of toxicology. It is a process that allows one to compare life table parameters for unexposed populations with those for populations exposed to various concentrations of a pollutant. Moreover, they explained that because of stricter regulations placed on insecticides requiring them to be more environmentally friendly, recently developed insecticides are slower acting and produce a greater degree of sublethal effects than earlier ones. Hence, the use of life tables combining both lethal and sublethal effects is expected to be beneficial in evaluating efficacy of insecticides.

Specifically, Stark and Wennergren studied the effects of the neem insecticide, Margosan-O on the pea aphid, Acyrthosiphon pisum by exposing both neonates and adults in separate sets of experiments. Twenty-one pots of broad bean, Vicia faba, were planted in rectangular pots $10 \mathrm{~cm}$ wide, $10 \mathrm{~cm}$ high, and grown in a greenhouse. When the plants reached around $25 \mathrm{~cm}$ in height, they were thinned to five per pot and treated with a water control or one of six concentrations of 
Margosan-O (10, 20, 40, 80, $100 \mathrm{ppm})$. Three pots of broad bean were treated for each concentration. The plants were treated completely on both the upper and under surfaces of all leaves and allowed to dry.

For the exposure of the neonates, one young apterous adult female aphid was placed in a clip cage attached to the leaves of the treated plants. There were ten clip cages per plant, thus ten aphids per pot and thirty per concentration. Because of the structure of the clip cages, the aphids were in constant contact with the surface of the leaves, and hence, chronically exposed. After 24 hours, all aphids were removed from the cages except one 1st instar. Thus these neonate aphids were exposed at birth to a specific treatment level and were then studied with mortality and reproduction recorded daily throughout their life-span.

Exposure of adult aphids was completed in a manner similar to that of the neonates. One young apterous adult female was placed in a clip cage attached to the leaves of untreated plants. There were ten clip cages per plant, for a total of 210 aphids. Again, all aphids except one 1st instar were removed from the cages. These aphids were kept in the cages until one day after they reached the adult stage. The aphid reproduces on day five or six of their life-span, thus they were kept in the cages until day seven. The adult aphids were relocated to a separate set of clip cages on broad bean plants that had been treated with the various concentrations of Margosan-O and the control. These adults were then studied with mortality and reproduction recorded daily. For a more detailed explanation of the experimental procedure see [SW].

Mortality and reproduction were recorded for exposure of both neonates and adults and thus the data collected is the population density at age, $x$, and time, $t$. Assuming we know the percent mortality and percent of loss of reproduction, we can calculate various parameters that are of interest. Stark and Wennergren used the intrinsic rate of increase $\left(r_{m}\right)$ as a bioassay parameter because it is based on survivorship $(L)$. These values can be found using the experimental data. All formulas pertaining to the Leslie model and life table parameters can be found in Table 1, and are discussed in more detail throughout the next sections.

\subsection{Model Description}

An age-structured matrix projection model known as the Leslie matrix model was used to investigate the population structure. The Leslie matrix model is a discrete model that uses age distributions to project population densities as time progresses. As discussed in $[\mathrm{K}]$, it was introduced by Whelpotn in 1936 and formalized by Bernardelli in 1941, Lewis in 1942, and Leslie in 1945 and 1948. The basic structure of the Leslie matrix is given in equation (1), 
Table 1: Formulas and descriptions for life table parameters

\begin{tabular}{|c|c|c|}
\hline Parameter & Physical Description & Formula \\
\hline$x$ & Age Interval in Days & $\mathrm{N} / \mathrm{A}$ \\
\hline$t$ & Number of Days Experiment was Run & $\mathrm{N} / \mathrm{A}$ \\
\hline$D(x)$ & Initial Adult Population & $\mathrm{N} / \mathrm{A}$ \\
\hline$d(x)$ & Initial Neonate Population & $\mathrm{N} / \mathrm{A}$ \\
\hline$N(t)$ & Population Density & $\mathrm{N} / \mathrm{A}$ \\
\hline mort & Percent Mortality & $\mathrm{N} / \mathrm{A}$ \\
\hline loss & Percent Loss of Reproduction & $\mathrm{N} / \mathrm{A}$ \\
\hline$S(x)$ & Surviving Number of Adults Alive & $\frac{D(x)(100-\text { mort })}{100}$ \\
\hline$s(x)$ & Surviving Number of Neonates Alive & $\frac{d(x)\left(100-\text { loss }^{2}(100-\text { mort })\right.}{100^{2}}$ \\
\hline$L(x)$ & Probability of Survival to Next Age Interval & $\frac{S(x)}{S(0)}$ \\
\hline$M(x)$ & Number of Offspring Per Surviving Female & $\frac{s(x)}{S(x)}$ \\
\hline$G(x)$ & Probability to Survive and Grow & $\frac{L(x+1)}{L(x)}$ \\
\hline$F(x)$ & Fecundity & $\frac{M(x)+G(x) * M(x+1)}{2}$ \\
\hline$R_{0}$ & Net Reproductive Rate & $\sum_{x} L(x) M(x)$ \\
\hline$T$ & Mean Generation Time & $\frac{\sum_{x} x L(x) M(x)}{\sum_{x} L(x) M(x)}$ \\
\hline$r_{m}$ & Intrinsic Rate of Increase & $\frac{\ln R_{0}}{T}$ \\
\hline$r_{i}$ & Instantaneous Rate of Increase & $\frac{\ln \left(\frac{N(t)}{N(0)}\right)}{t-1}$ \\
\hline$b$ & Intrinsic Birth Rate & $\frac{1}{\sum_{x} L(x) e^{-r_{m} x}}$ \\
\hline$d$ & Intrinsic Death Rate & $b-r_{m}$ \\
\hline
\end{tabular}

$$
L=\left[\begin{array}{ccccc}
F_{0} & F_{1} & F_{2} & \ldots & F_{\omega-1} \\
G_{0} & 0 & \ldots & \ldots & \ldots \\
0 & G_{1} & 0 & \ldots & \ldots \\
\ldots & \ldots & \ddots & \ddots & \ldots \\
0 & 0 & 0 & G_{\omega-2} & 0
\end{array}\right]
$$

with $F$ representing the fecundity, $G$ representing the probability to survive and grow into the next stage, and $\omega$ representing the number of stages. Fecundity and survivorship formulas for the pea aphid experiments are given in Table 1. They are based on percent mortality and percent loss reproduction values which were determined through calculations with the experimental data.

If one knows the age distribution or population density at age $x$ and time $t$, one can project to time $t+1$. Therefore, population growth can be found via repeated matrix multiplications as shown in equation (2). In vector matrix notation the model can be written as $n_{t+1}=L n_{t}$, where $n_{j, t}$ is the population density at age $j$ and time $t$, and $L$ is the Leslie matrix: 


$$
\left[\begin{array}{c}
n_{0, t+1} \\
n_{1, t+1} \\
\vdots \\
n_{\omega-1, t+1}
\end{array}\right]=\left[\begin{array}{ccccc}
F_{0} & F_{1} & F_{2} & \ldots & F_{\omega-1} \\
G_{0} & 0 & \ldots & \ldots & \ldots \\
0 & G_{1} & 0 & \ldots & \ldots \\
\ldots & \ldots & \ddots & \ddots & \ldots \\
0 & 0 & 0 & G_{\omega-2} & 0
\end{array}\right]\left[\begin{array}{c}
n_{0, t} \\
n_{1, t} \\
\vdots \\
n_{\omega-1, t}
\end{array}\right]
$$

Using our previous results, we can solve for the net reproductive rate $\left(R_{0}\right)$ and mean generation time $(T)$ given in Table 1 that are then used to calculate two population-level ecotoxicological endpoints: intrinsic rate of increase $\left(r_{m}\right)$ and direct instantaneous growth rate $\left(r_{i}\right)$. Both measurements are of interest to ecologists. The rate $\left(r_{m}\right)$ allows us to determine both the intrinsic birth and death rate, and the stable age distribution, all of which provide more insight into the population dynamics. The rate $\left(r_{i}\right)$ is determined using the results from the Leslie simulation.

\subsection{Simulations and Results}

To provide a basis for comparison of our results, we used MATLAB to recompute all of the Leslie simulations previously carried out by one of the authors (J Stark) of this work. Experimental data for the control experiment (no pesticide treatment) with aphids exposed as neonates are given in Table 2. This data was used, along with an assumed percent mortality and percent loss reproduction equivalent to zero, to solve for the parameters in Table 1. Once survivorship and fecundity were determined, we were able to develop the Leslie matrix.

Table 2: Control data for neonate exposure

\begin{tabular}{|c|c|c|c|c|c|c|c|c|}
\hline Age Class & $\mathbf{0}$ & $\mathbf{1}$ & $\mathbf{2}$ & $\mathbf{3}$ & $\mathbf{4}$ & $\mathbf{5}$ & $\mathbf{6}$ & $\mathbf{7}$ \\
\hline Total Alive & 30 & 30 & 30 & 30 & 30 & 30 & 30 & 30 \\
\hline Total Young & 0 & 0 & 0 & 0 & 0 & 0 & 9 & 118 \\
\hline Age Class & $\mathbf{8}$ & $\mathbf{9}$ & $\mathbf{1 0}$ & $\mathbf{1 1}$ & $\mathbf{1 2}$ & $\mathbf{1 3}$ & $\mathbf{1 4}$ & $\mathbf{1 5}$ \\
\hline Total Alive & 30 & 30 & 30 & 30 & 30 & 30 & 29 & 28 \\
\hline Total Young & 232 & 209 & 202 & 220 & 246 & 179 & 181 & 149 \\
\hline Age Class & $\mathbf{1 6}$ & $\mathbf{1 7}$ & $\mathbf{1 8}$ & $\mathbf{1 9}$ & $\mathbf{2 0}$ & $\mathbf{2 1}$ & $\mathbf{2 2}$ & $\mathbf{2 3}$ \\
\hline Total Alive & 28 & 28 & 28 & 28 & 28 & 28 & 27 & 27 \\
\hline Total Young & 133 & 116 & 100 & 54 & 46 & 27 & 7 & 5 \\
\hline Age Class & $\mathbf{2 4}$ & $\mathbf{2 5}$ & $\mathbf{2 6}$ & $\mathbf{2 7}$ & $\mathbf{2 8}$ & $\mathbf{2 9}$ & $\mathbf{3 0}$ & $\mathbf{3 1}$ \\
\hline Total Alive & 26 & 26 & 26 & 20 & 13 & 9 & 5 & 2 \\
\hline Total Young & 0 & 0 & 0 & 0 & 0 & 0 & 0 & 0 \\
\hline
\end{tabular}

Similar experimental data for all six concentrations of aphids exposed both as neonates and as adults was used to develop a Leslie matrix for each case. Percent mortality and percent loss reproduction values, which determine survivorship and fecundity, for each concentration can be 
found in Table 3 and in Figure 1. We see that the neonates were much more susceptible to higher concentrations of Margosan-O, than were the adults.

Table 3: Percent mortality and percent loss reproduction for neonate and adult exposure

\begin{tabular}{|c|cc|cc|}
\hline Concentration & \multicolumn{2}{|c|}{$\begin{array}{c}\text { Percent } \\
\text { Mortality }\end{array}$} & \multicolumn{2}{c|}{$\begin{array}{c}\text { Percent Loss } \\
\text { Reproduction }\end{array}$} \\
\hline \hline & Neonate & Adult & Neonate & Adult \\
\hline $10 \mathrm{ppm}$ & 7 & 17 & 59 & 23 \\
\hline $20 \mathrm{ppm}$ & 30 & 24 & 85 & 40 \\
\hline $40 \mathrm{ppm}$ & 70 & 52 & 97 & 50 \\
\hline $60 \mathrm{ppm}$ & 80 & 55 & 99 & 54 \\
\hline $80 \mathrm{ppm}$ & 95 & 55 & 100 & 65 \\
\hline $100 \mathrm{ppm}$ & 98 & 62 & - & 70 \\
\hline
\end{tabular}
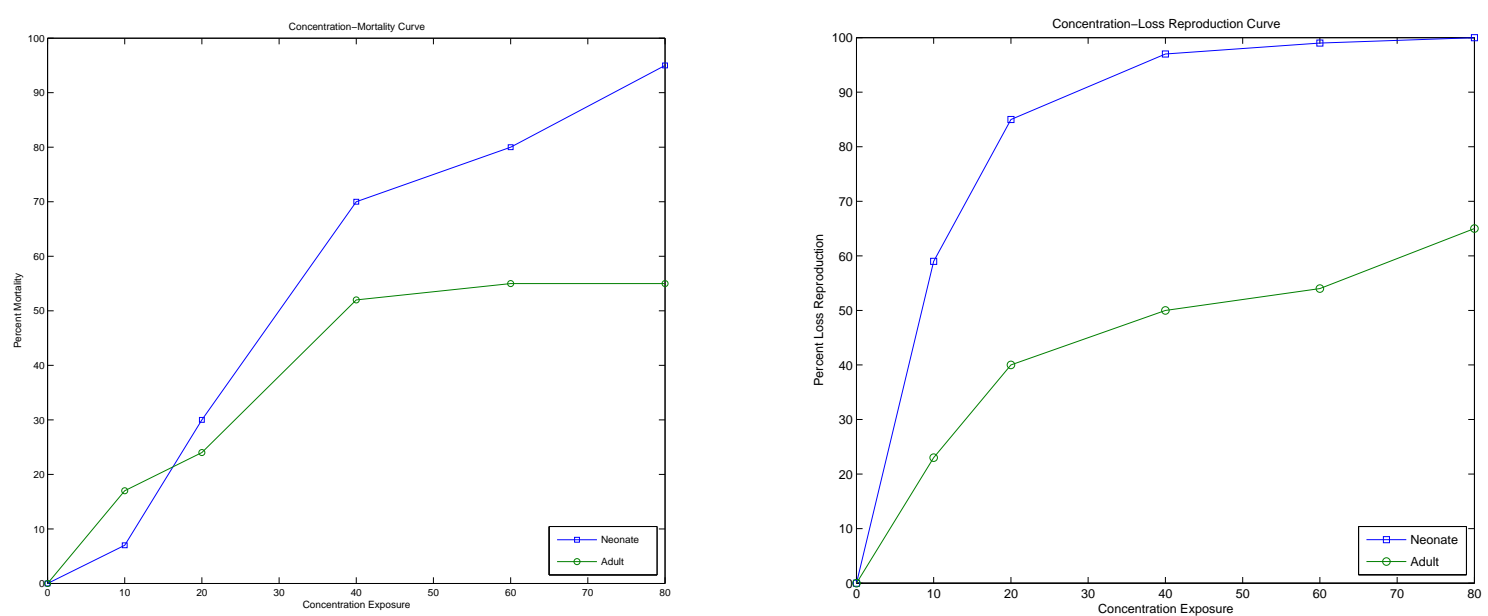

Figure 1: Percent mortality (left) and percent loss reproduction (right)

Survivorship graphs for the aphids in each age class, for both neonate and adult exposure, can be seen in Figure 2. Survivorship of aphids exposed to Margosan-O as neonates was reduced as concentration of Margosan-O increased. For an exposure of 80ppm, the maximum longevity of the neonates after exposure was five days compared with 32 days for the control. For aphids exposed as adults, there was a slight reduction of survivorship, but the effect was much less than in individuals exposed as neonates. For an exposure of $100 \mathrm{ppm}$, the maximum longevity of the aphids was 24 days compared with 32 days from the control. These results are supported by [RS]: the aphid population growth is significantly inhibited in a concentration-dependent manner by the introduction of Margosan-O. It should also be noted that under different concentrations, the effect 
of the insecticide declines as older aphids are exposed. This is supported by our simulations and the results presented in [WS].
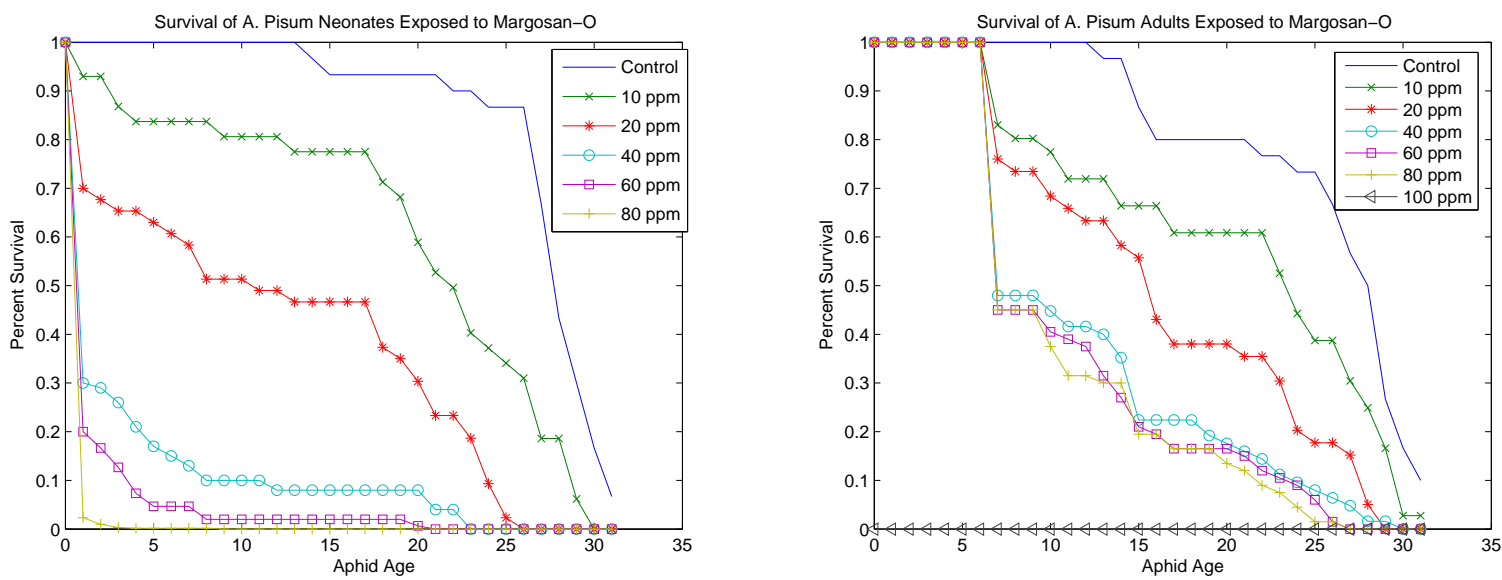

Figure 2: Survival of neonate exposure (left) and adult exposure (right)

The relationship between concentration and fecundity is similar to that of concentration and survivorship. Fecundity graphs for the aphids in each age class, both neonate and adult exposure, can be seen in Figure 3. Clearly, aphids exposed to Margosan-O as neonates undergo a huge decrease in their fecundity rates. For as little an exposure as 40ppm, the fecundity of the aphids is negligible. Maximum fecundity was around $80 \%$ for the control compared with a zero fecundity for pesticide concentrations higher than 40ppm. For aphids exposed as adults, while fecundity was reduced in a concentration dependent manner, the effect of the pesticide was not as prominent as for neonate exposure. Maximum fecundity was around $20 \%$ for an exposure of 100ppm compared with a maximum fecundity of around $90 \%$ for the control.

Once survivorship and fecundity were determined, simulations for each exposure method and concentration level were run beginning with day 0, and starting with 30 aphids in age class 0, 20 aphids in age class 1, 10 aphids in age class 2, 10 aphids in age class 3, 30 aphids in age class 4 and 0 aphids in the rest of the age classes. These aphids make up our initial age distribution vector that was then multiplied by the Leslie matrix to obtain our population projections. All simulations were run for 96 days. 

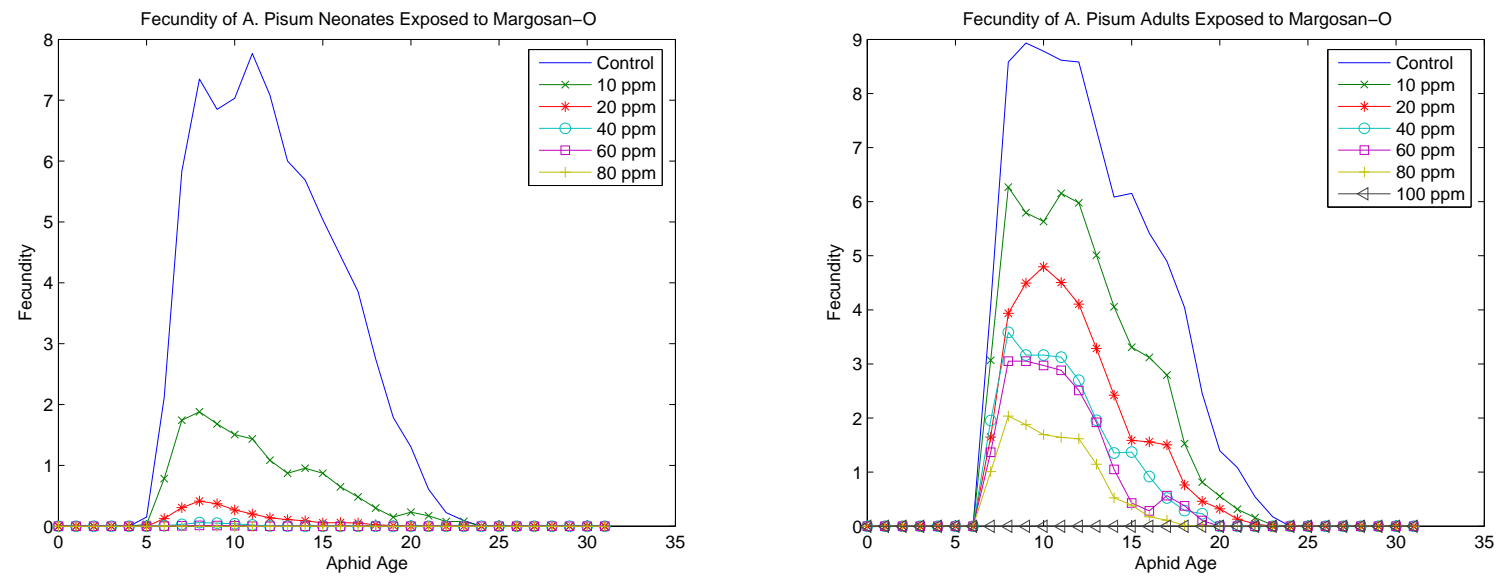

Figure 3: Fecundity of neonate exposure (left) and adult exposure (right)
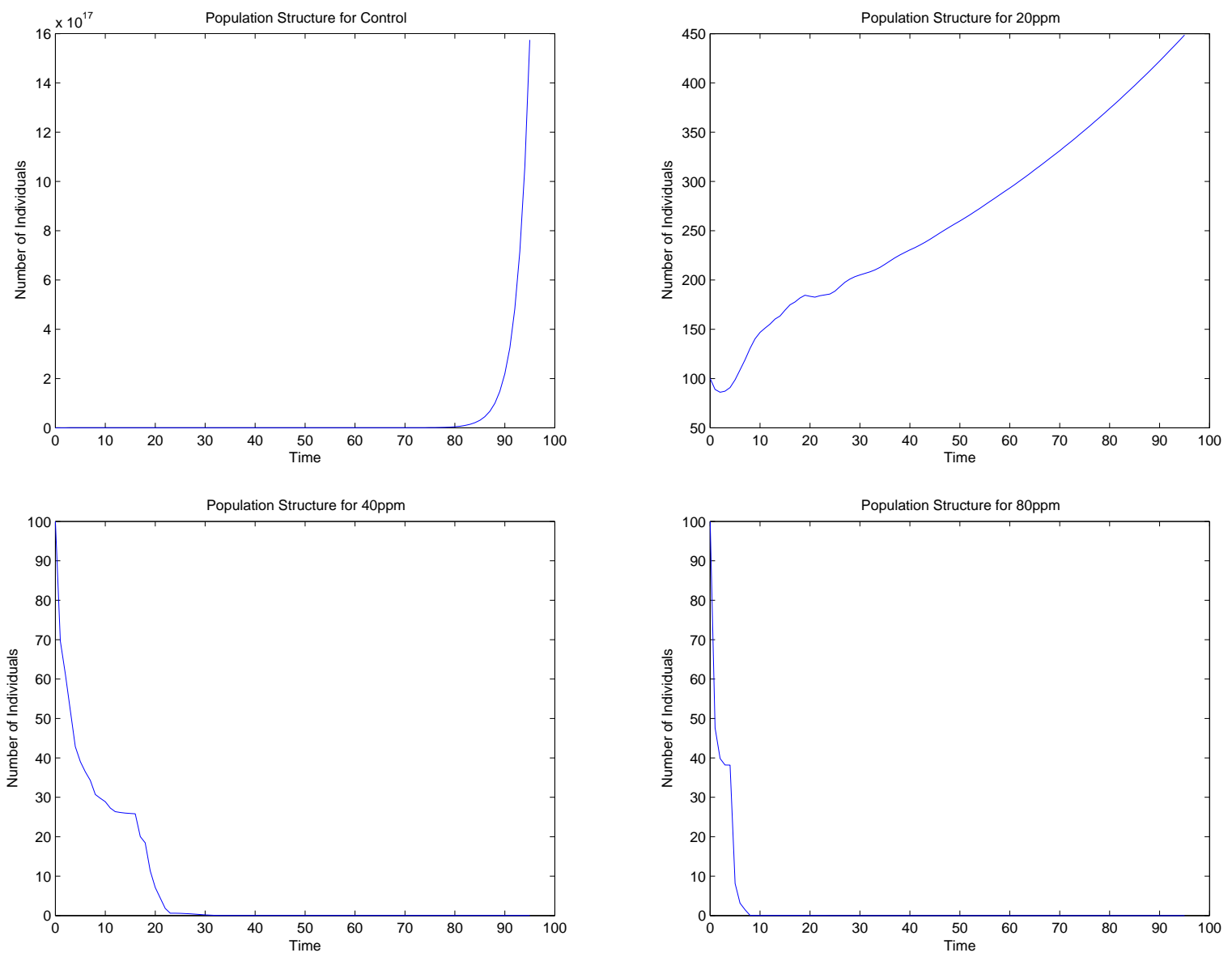

Figure 4: Neonate population growth-100 day simulation 
Some results for neonate exposure can be seen in Figure 4. The population growth for the control experiment appears exponential, which is what we would expect. However, once exposed to the pesticide, the population growth does not remain exponential. For neonate exposure, a concentration of 10ppm (not shown) still allows exponential growth, a concentration of 20ppm shows an eventually linear growth, and all higher concentrations show an exponential decline in population. The population level at day 96 for the control is $1.58 \times 10^{18}$ compared with 0 for an exposure of $80 \mathrm{ppm}$. For adult exposure, population growth remains exponential for all concentrations, however population maximums are reduced in a concentration dependent manner (see Figure 5). The population level at day 96 for the control is $5.46 \times 10^{17}$ compared with $4.82 \times 10^{7}$ for an exposure of 80ppm.
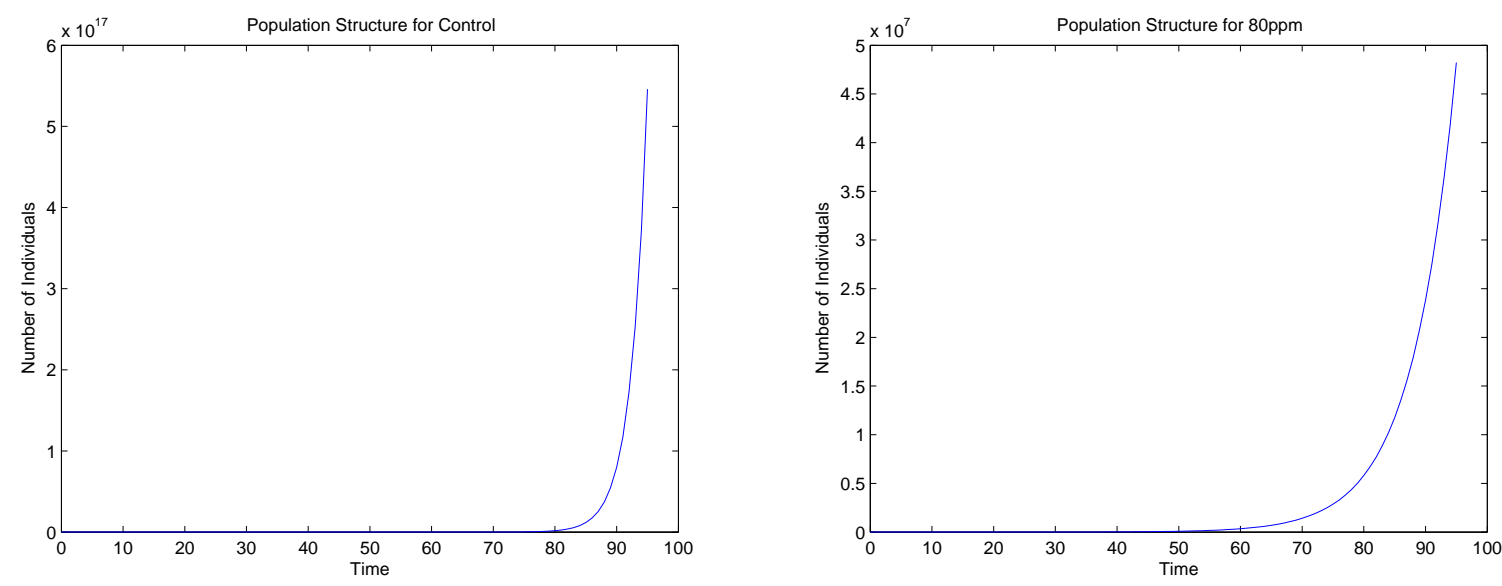

Figure 5: Adult population growth-100 day simulation

Using the results from the simulation, we are able to calculate the net reproductive rate $\left(R_{0}\right)$ and mean generation time $(T)$ for all concentrations and both means of exposure. Graphs of $R_{0}$ and $T$ can be found in Figure 6. According to [SW], the net reproductive rate is the number of females that replace the average female over a generation. We see that $R_{0}$ decreases as a function of Margosan-O concentration. For neonate exposure, the number of females that replaced an average female over a generation was 74.4 for the control compared with $6.6 \times 10^{-7}$ for $80 \mathrm{ppm}$ exposure. For adult exposure, the net reproductive rate was 81.9 for the control and 4.51 for $80 \mathrm{ppm}$ exposure. The reduction of $R_{0}$ was not as substantial with adult exposure, but the effect of the chemical can still be seen. Mean generation time $(T)$ also declined with increased pesticide concentration. The more pesticide to which the aphids were exposed, the lower the mean generation time. As might be expected, the aphids exposed from birth were affected more than the aphids exposed as adults for all pesticide concentrations.

Once we obtained the values of $R_{0}$ and $T$, we were able to determine the intrinsic rate of increase 

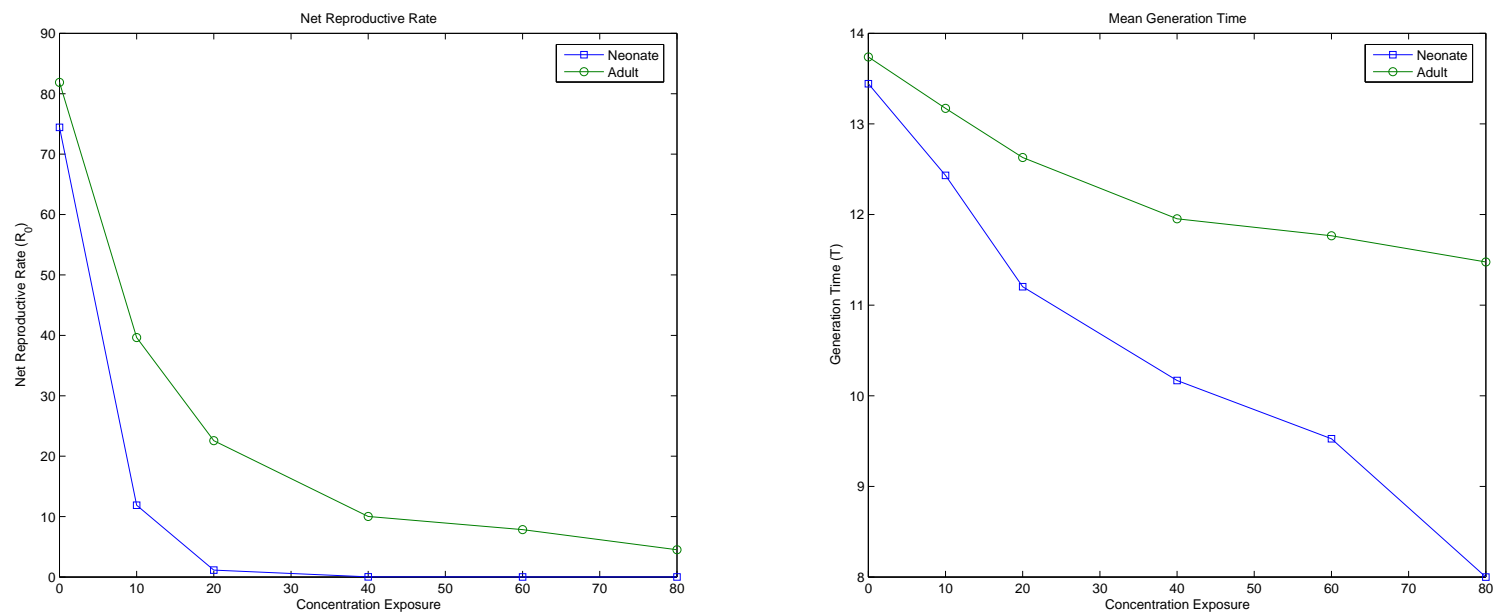

Figure 6: Net reproductive rate and mean generation time for neonates and adults

$\left(r_{m}\right)$ for each exposure case. As reported in [WS], the intrinsic rate of increase is a measure of the ability of a population to increase exponentially in an unlimited environment. Thus $r_{m}$ is an important measurement of population dynamics. This rate is given by $r_{m}=\frac{\ln R_{0}}{T}$. Our calculated $r_{m}$ values are depicted in Figure 7. For neonate exposure, $r_{m}$ decreased in a concentration dependent manner, becoming negative at 40ppm. A negative $r_{m}$ implies population extinction. Interestingly, for adult exposure, $r_{m}$ does not appear to be significantly affected by any amount of pesticide.

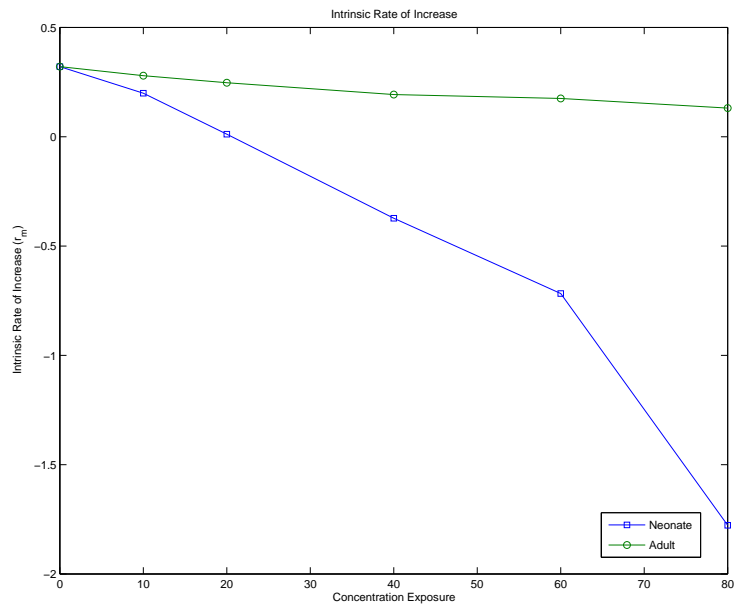

Figure 7: Intrinsic growth rate for neonates and adults

Ecologists have studied the differences between instantaneous rate of increase $\left(r_{i}\right)$ and the intrinsic rate of increase $\left(r_{m}\right)$. As explained in [WS], the instantaneous rate of increase is a measure 
of the ability of a population to increase exponentially over time. This rate is given by $r_{i}=r_{i}(t)=$ $\frac{1}{t-1} \ln \left(\frac{N(t)}{N(0)}\right)$. We see that there is a $r_{i}$ value for each day the simulation is run. Since we need a single value of $r_{i}$ to compare to $r_{m}$, we obtain our $\hat{r}_{i}$ value by finding the value equivalent to the population growth rate once the stable age distribution has been reached, which is just determining the asymptote. Figure 8 depicts the instantaneous direct measure of growth rate $\left(r_{i}\right)$ for neonate and adult exposure both as a population and daily. As with the $r_{m}$ values, a negative $r_{i}$ implies population extinction. Although an asymptote is not reached for neonate exposure of $80 \mathrm{ppm}$, we are not surprised by these results.
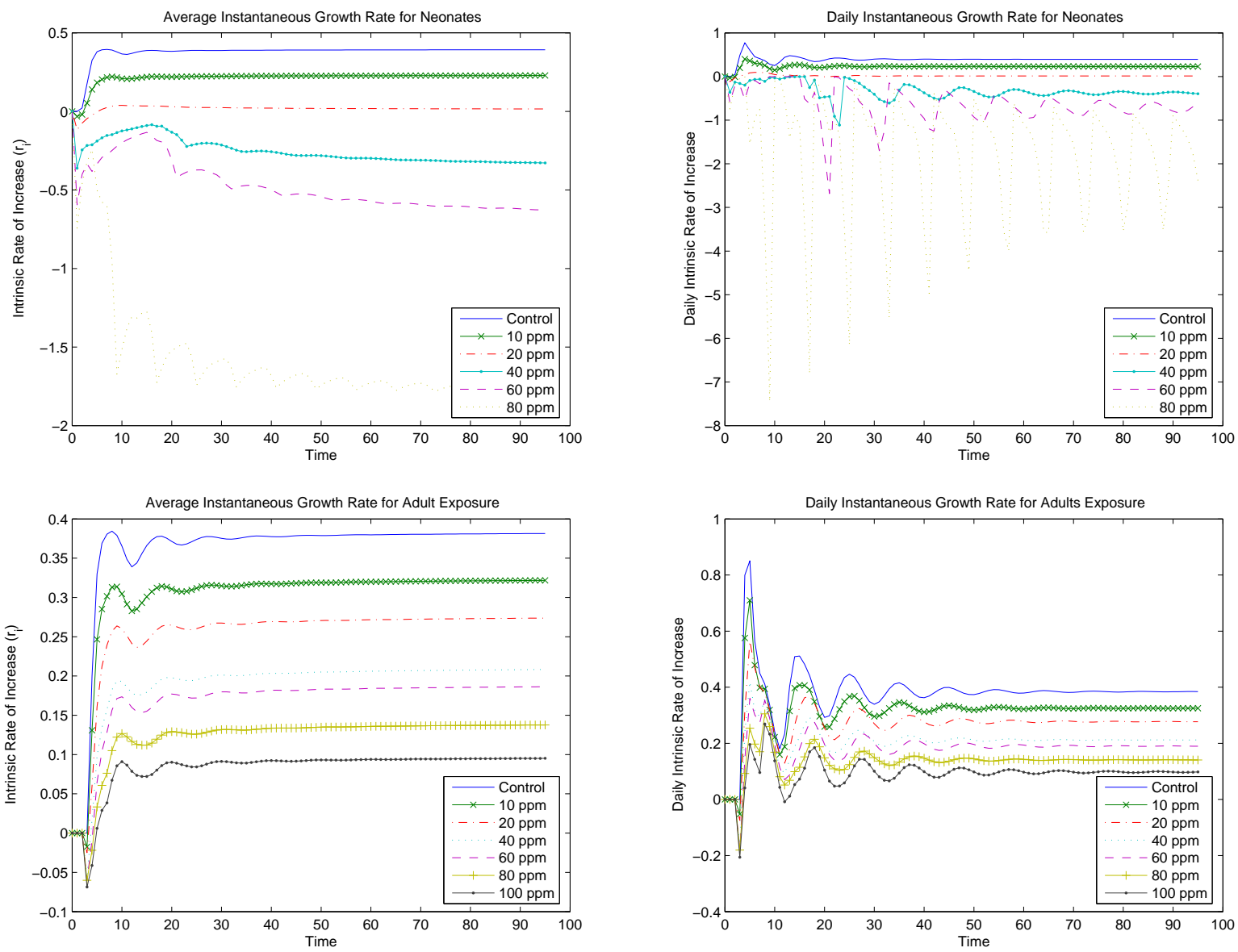

Figure 8: Direct instantaneous growth rates for neonate (top) and adult (bottom) exposure

The authors of [WS] suggested that $\hat{r}_{i}$ could be used as a substitute for $r_{m}$ as a population endpoint measurement. They reported no significant difference $(p=95 \%)$ between the two values. A p-value of $95 \%$ means (using conventional terminology) one is $95 \%$ confident that there is no difference between $r_{m}$ and $\hat{r}_{i}$. Our calculated $\hat{r}_{i}$ values are listed with the corresponding $r_{m}$ values in Table 4. As mentioned above, our $\hat{r}_{i}$ values should closely approximate our $r_{m}$ values; however, this 
does not always seem to be the case. One-way analysis of variance (ANOVA) was not performed on this data set, so we are not certain of the significance of the difference between $r_{m}$ and $\hat{r}_{i}$. Nevertheless, ecologists would like to use the $\hat{r}_{i}$ values instead of the $r_{m}$ values because $r_{m}$ is much more difficult to calculate.

Table 4: Intrinsic versus instantaneous rate of increase

\begin{tabular}{|c|cc|cc|}
\hline Concentration & \multicolumn{2}{|c|}{$\begin{array}{c}\text { Neonate } \\
\text { Exposure }\end{array}$} & \multicolumn{2}{c|}{$\begin{array}{c}\text { Adult } \\
\text { Exposure }\end{array}$} \\
\hline \hline & $\hat{r}_{i}$ & $r_{m}$ & $\hat{r}_{i}$ & $r_{m}$ \\
\hline Control & .393 & .321 & .381 & .321 \\
\hline $10 \mathrm{ppm}$ & .229 & .199 & .322 & .280 \\
\hline $20 \mathrm{ppm}$ & .016 & .012 & .274 & .247 \\
\hline $40 \mathrm{ppm}$ & -.328 & -.373 & .208 & .193 \\
\hline $60 \mathrm{ppm}$ & -.627 & -.717 & .186 & .175 \\
\hline $80 \mathrm{ppm}$ & -1.768 & -1.778 & .138 & .131 \\
\hline $100 \mathrm{ppm}$ & - & - & .098 & .092 \\
\hline
\end{tabular}

With our $r_{m}$ value, or theoretically our $\hat{r}_{i}$ value, we can calculate our final parameters of interest. Graphs of the intrinsic birth and death rates for each concentration level and exposure method can be found in Figure 9. Birth rate decreased as concentration of pesticide increased for both neonate and adult exposure, but not surprisingly, the decrease was much more considerable for the neonate. Death rate increased as concentration of pesticide increased for neonate exposure, while, an increase in pesticide concentration did not appear to affect the death rate for adult exposure.
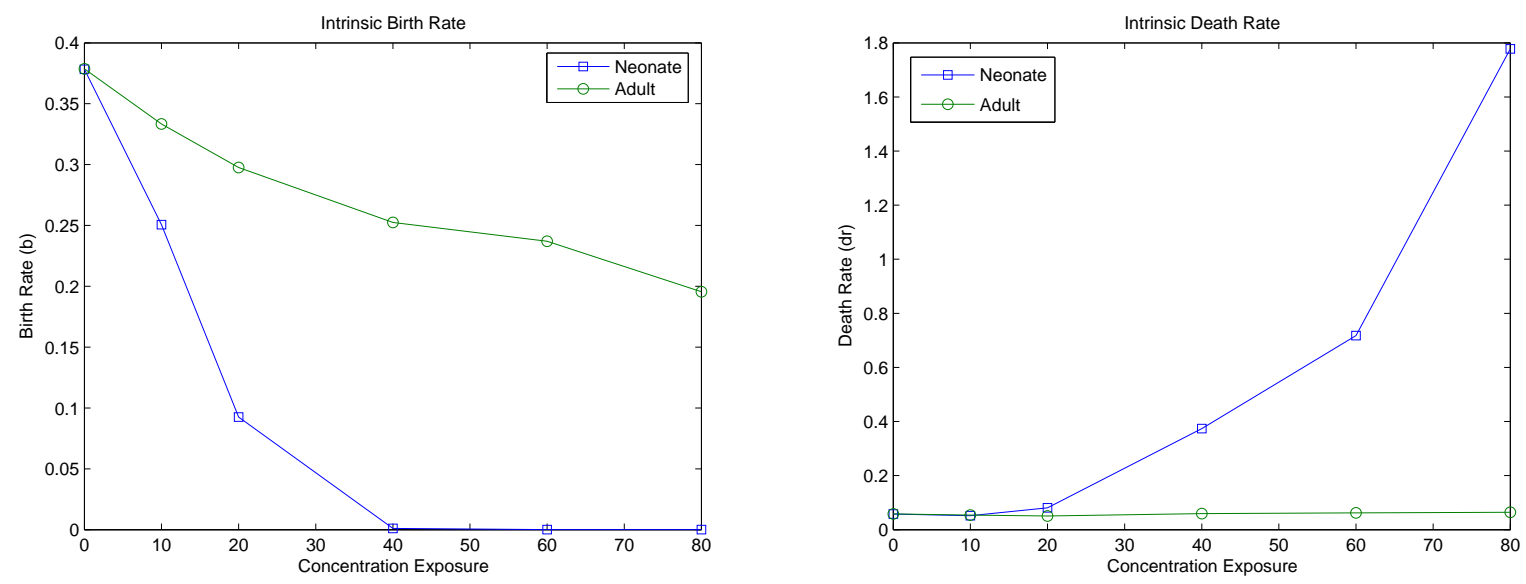

Figure 9: Intrinsic birth and death rates for neonates and adults 


\subsection{Time Dependence}

There is reason to believe that dealing with sublethal damage due to insecticide exposure generates a need for age/time dependent parameters such as mortality, birth and fecundity rates. It does not seem reasonable to assume that aphids will exhibit the same death rate one day after the spray and twenty days after the spray. In order to obtain time or age dependent parameters from the Leslie model, one must consider some of the input parameters to be time/age dependent. Since we are concerned with the death rate, we will consider percent mortality, mort $(x)$ to be age dependent. The formulas and descriptions for the life table parameters are given in Table 1.

Before we completely work out the formulas with $\operatorname{mort}(x)$, we first recall the expanded formulas with a constant mort:

$$
\begin{aligned}
S(x) & =\frac{D(x)(100-\text { mort })}{100} \\
s(x) & =\frac{d(x)(100-\text { loss })(100-\text { mort })}{100^{2}} \\
L(x) & =\frac{D(x)(100-\text { mort })}{100 D(1)} \\
M(x) & =\frac{d(x)(100-\text { loss })}{100 D(x)} \\
G(x) & =\frac{D(x+1)}{D(x)} \\
F(x) & =\frac{d(x) D(x+1)(100-\text { loss })+D(x) D(x+2) d(x+1)(100-\text { loss })}{200 D(x) D(x+1)},
\end{aligned}
$$

which result in the endpoint parameters:

$$
\begin{aligned}
R_{0} & =\frac{(100-\text { mort })(100-\text { loss })}{100^{2} D(1)} \sum_{x} d(x) \\
T & =\sum_{x} \frac{x d(x)}{\sum_{x} d(x)} \\
r_{m} & =\frac{\ln \left(\frac{(100-\text { mort })(100-\text { loss })}{100^{2} D(1)} \sum_{x} d(x)\right)}{\sum_{x} \frac{x d(x)}{d(x)}} \\
b & =\left(\frac{100-\text { mort }}{100 D(1)} \sum_{x} D(x) \exp \left(-x \frac{\ln \left(\frac{(100-\text { mort })(100-\text { loss })}{100^{2} D(1)} \sum_{x} d(x)\right)}{\sum_{x} \frac{x d(x)}{d(x)}}\right)\right)^{-1} \\
d & =b-r_{m} .
\end{aligned}
$$


Substituting mort $(x)$ into $S(x), s(x), L(x), M(x), G(x)$ and $F(x)$, we obtain

$$
\begin{aligned}
S(x) & =\frac{D(x)(100-\operatorname{mort}(x))}{100} \\
s(x) & =\frac{d(x)(100-\text { loss })(100-\operatorname{mort}(x))}{100^{2}} \\
L(x) & =\frac{D(x)(100-\operatorname{mort}(x))}{100 D(1)} \\
M(x) & =\frac{d(x)(100-\text { loss })}{100 D(x)} \\
G(x) & =\frac{D(x+1)(100-\operatorname{mort}(x+1))}{D(x)(100-\operatorname{mort}(x))} \\
F(x) & =\frac{d(x)(100-\text { loss })}{200 D(x)}+\frac{D(x+2) d(x+1)(100-\operatorname{mort}(x+2))(100-\text { loss })}{200 D(x+1)^{2}(100-\operatorname{mort}(x+1))} .
\end{aligned}
$$

with the first entry given by

$$
\begin{aligned}
S(1) & =D(1) \\
L(1) & =1 \\
M(1) & =\frac{d(1)(100-\operatorname{loss})(100-\operatorname{mort}(1))}{100^{2} D(1)} \\
G(1) & =\frac{D(2)(100-\operatorname{mort}(2))}{100 D(1)} \\
F(1) & =\frac{d(1)(100-\operatorname{loss})(100-\operatorname{mort}(1))}{200^{2} D(1)}+\frac{D(3) d(2)(100-\operatorname{mort}(3))(100-\text { loss })}{200 D(2)^{2}(100-\text { mort }(2))} .
\end{aligned}
$$

We see that the above formulas are more complicated than those with a constant mort. The endpoint parameters that result from the Leslie simulation are then:

$$
\begin{aligned}
R_{o} & =\frac{100-\text { loss }}{100^{2} D(1)} \sum_{x} d(x)(100-\operatorname{mort}(x)) \\
T & =\sum_{x} \frac{x d(x)(100-\operatorname{mort}(x))}{\sum_{x} d(x)(100-\operatorname{mort}(x))} \\
r_{m} & =\frac{\ln \left(\frac{100-\operatorname{loss}}{100^{2} D(1)} \sum_{x} d(x)(100-\operatorname{mort}(x))\right)}{\sum_{x} \sum_{x} d(x)(100-\operatorname{mort}(x))} \\
b & =\left(\sum_{x} \frac{D(x)(100-\operatorname{mort}(x))}{100 D(1)} \operatorname{mort}(x)\right) \\
d & =b-r_{m} .
\end{aligned}
$$

To illustrate a very simple age/time dependent mortality, we consider mort $(x)$ to be the piecewise constant function

$$
\operatorname{mort}(x) \approx \operatorname{mort}^{M}(x)=\sum_{j=1}^{M} a_{j} \chi_{j}^{M}(t)
$$


where $x_{j}^{M}=\frac{j x_{\max }}{M}$ for $j=0,1, \ldots, M$ and

$$
\chi_{j}^{M}= \begin{cases}1 & {\left[x_{j-1}^{M}, x_{j}^{M}\right)} \\ 0 & \text { elsewhere }\end{cases}
$$

If for example, we let $M=4$ and look at an example with 32 data points, then we will have

$$
\operatorname{mort}(x)= \begin{cases}a_{1} & {[0,8)} \\ a_{2} & {[8,16)} \\ a_{3} & {[16,24)} \\ a_{4} & {[24,32)}\end{cases}
$$

Thus our $R_{o}, T, r_{m}, b$ and $d$ will also be piecewise linear. We will consider each of the summations over the intervals listed in equation (3):

$$
\begin{aligned}
& R_{0}= \begin{cases}\frac{100-\operatorname{loss}\left(100-a_{1}\right)}{100^{2} D(1)} \sum_{x} d(x) & {[0,8)} \\
\frac{100-l o s s\left(100-a_{2}\right)}{100^{2} D(1)} \sum_{x} d(x) & {[8,16)} \\
\frac{100-l o s s\left(100-a_{3}\right)}{100^{2} D(1)} \sum_{x} d(x) & {[16,24)} \\
\frac{100-l o s s\left(100-a_{4}\right)}{100^{2} D(1)} \sum_{x} d(x) & {[24,32)}\end{cases} \\
& T= \begin{cases}\sum_{x} \frac{x d(x)\left(100-a_{1}\right)}{\sum_{x} d(x)\left(100-a_{1}\right)} & {[0,8)} \\
\sum_{x} \frac{x d(x)\left(100-a_{2}\right)}{\sum_{x} d(x)\left(100-a_{2}\right)} & {[8,16)} \\
\sum_{x} \frac{x d(x)\left(100-a_{3}\right)}{\sum_{x} d(x)\left(100-a_{3}\right)} & {[16,24)} \\
\sum_{x} \frac{x d(x)\left(100-a_{4}\right)}{\sum_{x} d(x)\left(100-a_{4}\right)} & {[24,32)}\end{cases} \\
& r_{m}= \begin{cases}\frac{\ln \left(\frac{100-\text { loss }}{100^{2} D(1)} \sum_{x} d(x)\left(100-a_{1}\right)\right)}{\sum_{x} \sum_{x} d(x)\left(100-a_{1}\right)} & {[0,8)} \\
\left.\frac{\ln \left(\frac{100-\operatorname{loss}}{100^{2} D(1)} \sum_{x} d(x)\left(100-a_{1}\right)\right.}{\sum_{x} \sum_{x} d(x)\left(100-a_{2}\right)}\right) & {[8,16)} \\
\left.\frac{\ln \left(\frac{100-\operatorname{loss}}{100^{2} D(1)} \sum_{x} d(x)\left(100-a_{2}\right)\right.}{\sum_{x} \sum_{x} d(x)\left(100-a_{3}\right)}\right) & {[16,24)} \\
\left.\frac{\ln \left(\frac{100-\operatorname{loss}}{100^{2} D(1)} \sum_{x} d(x)\left(100-a_{3}\right)\right.}{\sum_{x} d(x)\left(100-a_{4}\right)}\right) & {[24,32)}\end{cases}
\end{aligned}
$$




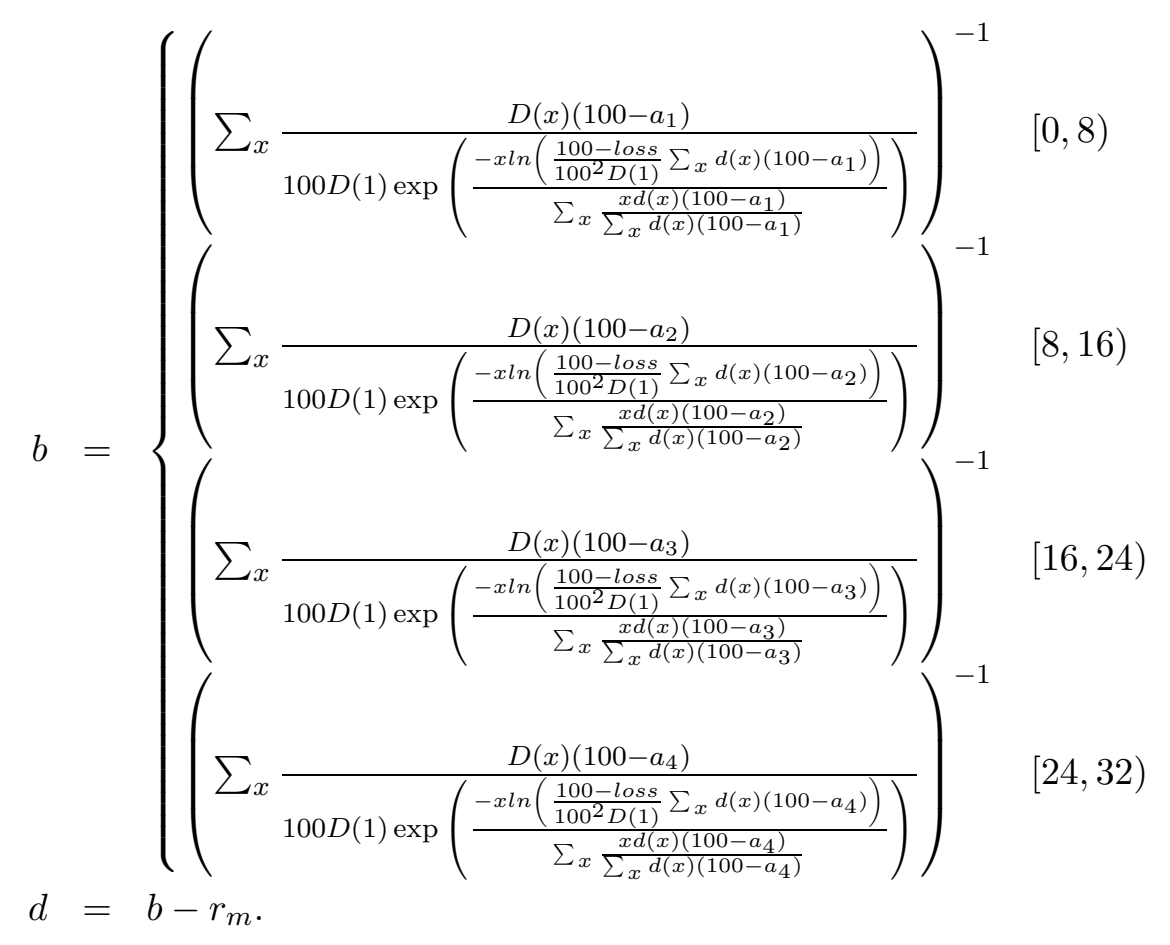

The resulting formulation of the Leslie matrix is much more complicated than when we considered all of our parameters to be constant. According to Wood [Wo2], "Although it is relatively easy to collect population data structured by life-history stage, the corresponding birth and death rates are all but impossible to measure directly." We see that obtaining the sublethal parameters of interest is a daunting task when using the Leslie model. Using parameter estimation techniques as we do in subsequent sections with a continuous model to estimate the rates $\left\{a_{i}\right\}$ would be extremely difficult if not impossible with the Leslie formulation.

In order to run simulations using the new formulation, one would in essence have to compute four different Leslie simulations. Wood [Wo3] comments on this idea of a parameter dependent stage-structured model, "While representing an advance on previous methods, the technique has several problems: time-dependent rates are only obtained by considering data in small sections and performing an analysis for each one." He continues on to explain why making a discrete or stage-structured model parameter dependent is not a good idea. He says, "There are fundamental demographic instabilities associated with estimating time and stage-dependent mortality rates from stage-structured data. The most important of these instabilities is that, even with perfect data, any small error in estimated mortality rates in one stage causes increasingly large errors in the mortality estimates for subsequent stages." Wood says the only way to get past the instability is to "make restrictive assumptions about underlying population dynamics," such as assuming one has constant parameters. Since we do not want to make this assumption, we turn in the next section to a different population model, one that allows for parameter-dependent parameters.

In his book [C], Caswell states that although they can be more difficult to work with, PDE's 
can describe the vital rates in detail, because they neither lump individuals into discrete classes nor divide time into intervals. We will be using the Sinko-Streifer or McKendrick and VonForester continuous age-structured population model. Ohman and Wood $[\mathrm{OH}]$ praise this model, remarking that "This method has been shown to be robust to moderate levels of sampling error and to avoid the demographic instabilities common to some other methods as well as to generate both time- and stage-dependent mortalities with confidence limits."

\section{Continuous Age-Structured Model}

We turn to an alternative approach to obtaining information on sublethal damage from insecticide treatments. In doing so, we wish to extrapolate information from the Leslie models and incorporate it into a continuous age-structured model. McKendrick and VonForester (see [K] for a historical summary) worked with age structured models [M,VF] and were the first (1959) to develop the wellknown partial differential equation (PDE), continuous-time model, which was later popularized by Sinko and Streifer (1967). The Sinko-Streifer model [SS] made allowances for size/age dependence growth rates. In this seminal effort, Sinko-Streifer (SS) worked with size-structured populations to describe population dynamics.

The Sinko-Streifer population model involves growth and mortality with size and/or age dependent parameters. We will work with the age-structured population model in an attempt to describe the population dynamics of aphids exposed to different concentrations of Margosan-O. Under specific assumptions on recruitment, we derive the exact solution of the model using the Method of Characteristics, considering the population structure as a function of age $(x)$ and time $(t)$. We then utilize information gathered from Leslie to run simulations.

\subsubsection{Model Assumptions}

Before discussing the formulation of the model, we will discuss the underlying assumptions as presented in $[\mathrm{BT}]$. First, the age growth rate (denoted $g$ ) of same sized individuals are the same. Thus

$$
\frac{d x}{d t}=g(x, t)
$$

Second, we assume that there is a "minimum" and a "maximum" age, or equivalently

$$
x_{0} \leq x \leq x_{\max }
$$

Third, the death rate (denoted by $\mu$ ) is assumed to be proportional to the age density, while the recruitment rate $(R)$ is assumed to be proportional to the population age density. Finally, we assume that the population is sufficiently large so as to allow us to use a continuous model to approximate the population densities. 


\subsubsection{Sinko-Streifer Model}

With these assumptions in mind, we write the model:

$$
\frac{\partial u}{\partial t}+\frac{\partial}{\partial x} g u=-\mu u \text { with } x \in\left(x_{0}, x_{\max }\right) \text { and } t \in\left(0, t_{f}\right),
$$

with a boundary condition

$$
R(t)=g\left(x_{0}, t\right) u\left(x_{0}, t\right)=\int_{x_{0}}^{x_{\max }} k(\xi, t) u(\xi, t) d \xi
$$

and an initial condition

$$
u(x, 0)=\Phi(x),
$$

where $u(x, t)$ represents the population density at age $x$ and time $t, g(x, t)$ represents the individual growth or aging rate, $\Phi(x)$ represents the initial age density, and $k$ represents the fecundity rate. We also have $x_{\max }$ equal to the maximum age, while $x_{0}$ is the initial age. As stated in the assumptions, $R$ is the recruitment rate, $\Phi$ is the initial age density, and the death rate is $\mu$.

\subsubsection{Derivation Via Method of Characteristics}

In order to solve equation (4), we reduce the partial differential equation (PDE) to ordinary differential equations (ODEs). We do this using the method of characteristics discussed in [BT]. We note that for our specific problem, the growth or aging rate is given by $g(x, t)=1$. If an aphid in age class $x$ is alive at time $t$, she will always move into the next age class $x+1$ at time $t+1$. Therefore we compute the exact solution $u(x, t)$ for the case of constant growth rate $(g(x, t)=1)$ and death rate $\left(\mu(x, t)=\mu_{0}\right)$. The general form of the solution has two parts, one initial condition driven and the other boundary condition driven. Since $g(x, t)$ is constant, these conditions depend solely on the relationship between age $(x)$ and time $(t)$. The initial condition driven solution is found when $t \leq G(x)$ and is given by

$$
u(x, t)=\Phi(X(0 ; x, t)) e^{-\int_{0}^{t}\left[g_{x}(\xi, X(\xi ; x, t))+\mu(\xi, X(\xi ; x, t))\right] d \xi},
$$

while the boundary condition driven solution holds when $t>G(x)$ and is given by

$$
u(x, t)=\frac{R\left(T\left(x_{0} ; x, t\right)\right)}{g\left(T\left(x_{0} ; x, t\right), x_{0}\right)} e^{-\int_{T\left(x_{0} ; x, t\right)}^{t}\left[g_{x}(\xi, X(\xi ; x, t))+\mu(\xi, X(\xi ; x, t))\right] d \xi} .
$$

Here $G(x)$ is defined as $G(x)=T\left(x ; 0, x_{0}\right)$, where $(x, G(x))$ is the characteristic curve that passes through $\left(x_{0}, 0\right)$. We must find the solution for $x$ and $t$ along characteristic curves: $X(0 ; x, t)$ is the characteristic curve evaluated at $t=0$ that passes through the point $(x, t)$, and $T\left(x_{0} ; x, t\right)$ is the characteristic curve evaluated at $x=x_{0}$ that passes through the point $(x, t)$. See Figure 10 for a graphical depiction of solution domain. 


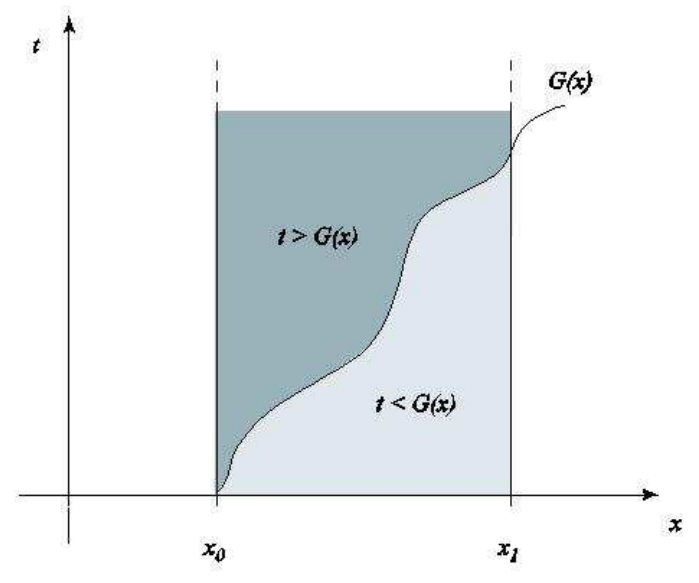

Figure 10: Characteristics

In order to compute the exact solution for our special case of $g=1$ and $\mu$ constant, we begin by computing: $X(t ; \hat{x}, \hat{t}), T(t ; \hat{x}, \hat{t})$ and $G(x)=T\left(x ; x_{0}, 0\right)$. We first consider $X(t ; \hat{x}, \hat{t})$ noting that $\frac{\partial x}{\partial t}=1$ and $x(\hat{t})=\hat{x}$. Thus $x(t)=t+c \Rightarrow c=\hat{x}-\hat{t}$. Using the previous results we have

$$
X(t ; \hat{x}, \hat{t})=t+\hat{x}-\hat{t} .
$$

Next solving for $\mathrm{T}$, we find

$$
T(t ; \hat{x}, \hat{t})=x-\hat{x}+\hat{t}
$$

Finally, we solve for $\mathrm{G}$ by substitution:

$$
G(x)=T\left(x ; x_{0}, 0\right)=x-x_{0} .
$$

Completing our special solution, we now substitute $X(t ; \hat{x}, \hat{t}), T(t ; \hat{x}, \hat{t})$ and $G(x)=T\left(x ; x_{0}, 0\right)$ into equations (6) and (7). We begin with the initial condition driven solution (i.e., $t \leq G(x)$ ):

$$
\begin{aligned}
u(x, t) & =\Phi(X(0 ; x, t)) e^{-\int_{0}^{t}\left[g_{x}(\xi, X(\xi ; x, t))+\mu(\xi, X(\xi ; x, t))\right] d \xi} \\
& =\Phi(x-t) e^{-\int_{0}^{t}\left(0+\mu_{0}\right) d \xi} \\
& =\Phi(x-t) e^{-\left.\mu_{0} \xi\right|_{0} ^{t}} \\
& =\Phi(x-t) e^{-\mu_{0} t}
\end{aligned}
$$

and then the boundary condition driven solution $(t>G(x))$ :

$$
\begin{aligned}
u(x, t) & =\frac{R\left(T\left(x_{0} ; x, t\right)\right)}{g\left(T\left(x_{0} ; x, t\right), x_{0}\right)} e^{-\int_{T\left(x_{0} ; x, t\right)}^{t}\left[g_{x}(\xi, X(\xi ; x, t))+\mu(\xi, X(\xi ; x, t))\right] d \xi} \\
& =R\left(x_{0}-x+t\right) e^{-\int_{x_{0}-x+t}^{t}\left(0+\mu_{0}\right) d \xi} \\
& =R\left(x_{0}-x+t\right) e^{-\left.\mu_{0} \xi\right|_{x_{0}-x+t} ^{t}} \\
& =R\left(x_{0}-x+t\right) e^{\left(x_{0}-x\right) \mu_{0}} .
\end{aligned}
$$


Thus depending on the relationship between time, $t$, and growth rate, $g$, we have solved for an exact solution using the Method of Characteristics. Note that this requires the recruitment rate $R$ to be known or at least to be represented by some time dependent function $R(t)$.

\subsection{Simulations and Results}

In order to adapt the SS model to our specific problem, we have to first determine parameter values. We determined intrinsic death rates for each concentration and exposure type when running the Leslie simulations. The graphs of these values can be seen in Figure 9. The actual values that are substituted into our solutions are located in Table 5. Our age intervals range from age $x_{0}=1$ to age $x_{\max }=32$ and are measured in increments corresponding to days. (Note: We started Leslie with a zero age class (i.e., $x_{0}=0$ ). Here with SS we take $x_{0}=1$. This in no way affects our ability to compare results between the two models.) The simulations can be run for as many days as desired, but all simulations begin with day $t_{0}=0$ to facilitate comparison with the Leslie simulations. There were technical reasons for starting Leslie with a zero class pertaining to the manner in which our experimental data was given. With all of the previous parameters as known values, we are only left with determining our initial age distribution function and our recruitment function.

Table 5: Intrinsic death rates for neonate and adult exposures

\begin{tabular}{|c|c|c|}
\hline Concentration & Neonate Death Rate $\mu_{0}$ & Adult Death Rate $\mu_{0}$ \\
\hline \hline Control & .0576 & .0581 \\
\hline $10 \mathrm{ppm}$ & .0516 & .0540 \\
\hline $20 \mathrm{ppm}$ & .0810 & .0509 \\
\hline $40 \mathrm{ppm}$ & .37388 & .0597 \\
\hline $60 \mathrm{ppm}$ & .7174 & .0619 \\
\hline $80 \mathrm{ppm}$ & 1.7778 & .0643 \\
\hline
\end{tabular}

The initial age distribution function was determined using the initial data from the Leslie simulations. These values: 30 aphids in age class 0 (i.e., $x_{0}=1$ ), 20 aphids in age class 1,10 aphids in age class 2, 10 aphids in age class 3,30 aphids in age class 4 and 0 aphids in the rest of the age classes were placed into a vector and then linear interpolation was used to obtain our initial age distribution function.

Linear interpolation uses piecewise linear splines to represent a linear polynomial between two adjacent data points, thereby producing a piecewise smooth, continuous model for what was before discrete. The representation used to determine the initial age distribution function $\Phi(x)$ is given by 


$$
\Phi(x)=\sum_{j=1}^{6} \Phi_{j} l_{j}(x),
$$

with $\Phi_{j}$ representing the discrete values gathered from Leslie, and $l_{j}(x)$ representing the linear spline basis elements known as 'hat' functions. The 'hat' functions can be seen in Figure 11.

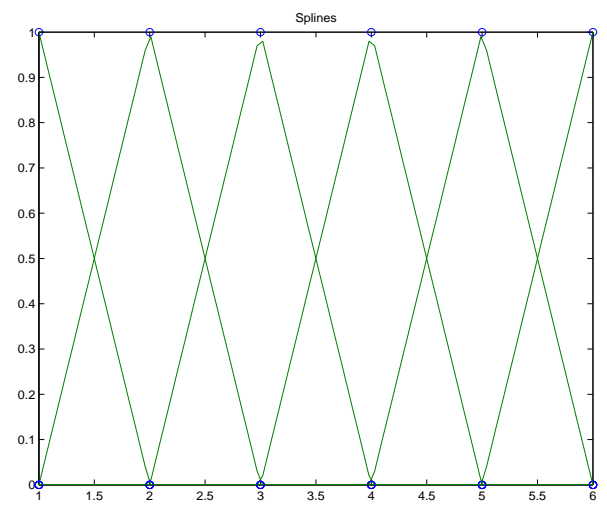

Figure 11: Linear spline 'hat' function

The calculated function $\Phi(x)$ is depicted in Figure 12. The value of $\Phi(x)$ for $x>6$ was taken as zero, because no individuals greater than six days old were in the initial population.

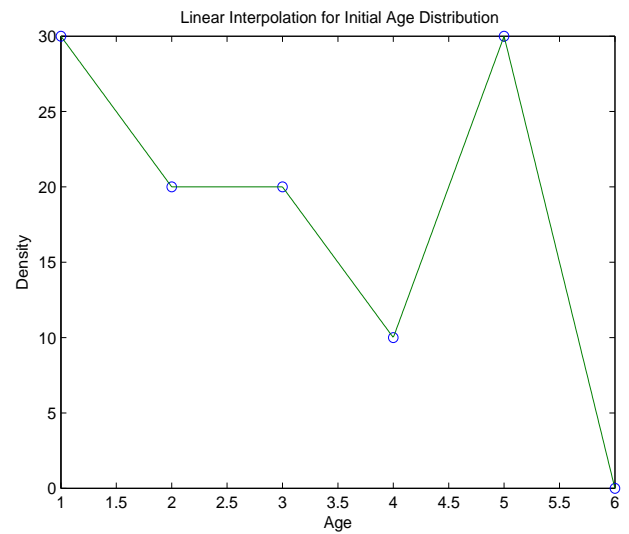

Figure 12: Initial age distribution function $\Phi(x, t)$ from $x=1$ to $x=6$

This same interpolation method was employed to determine the recruitment functions $R(t)$ for each exposure method and each concentration level. The first entry in each Leslie simulation represents the number of individuals being born into age class $x_{0}=1$ at each time increment, thus these entries can be thought of as the recruitment rate for the specific data set. The values from 
the first entry in each Leslie simulation were obtained, placed into a recruitment vector, and then linear interpolation was used to calculate the recruitment functions. The resulting functions can be seen in Figures 13 through 20.

Because we now have each necessary parameter to solve SS, we were able to run simulations for each exposure method and concentration level for a total of twelve SS simulations. Thus our SS model for the case of $g=1$ and constant $\mu=\mu_{0}$ is given by

$$
\begin{aligned}
& u(x, t)=\Phi(x-t) e^{-\mu_{0} t} \text { for } t \leq G(x) \\
& u(x, t)=R\left(x_{0}-x+t\right) e^{\left(x_{0}-x\right) \mu_{0}} \text { for } t>G(x) .
\end{aligned}
$$

\subsubsection{Neonate Exposure}

Results from neonate exposure for the control and 10ppm concentrations can be seen in Figure 13.
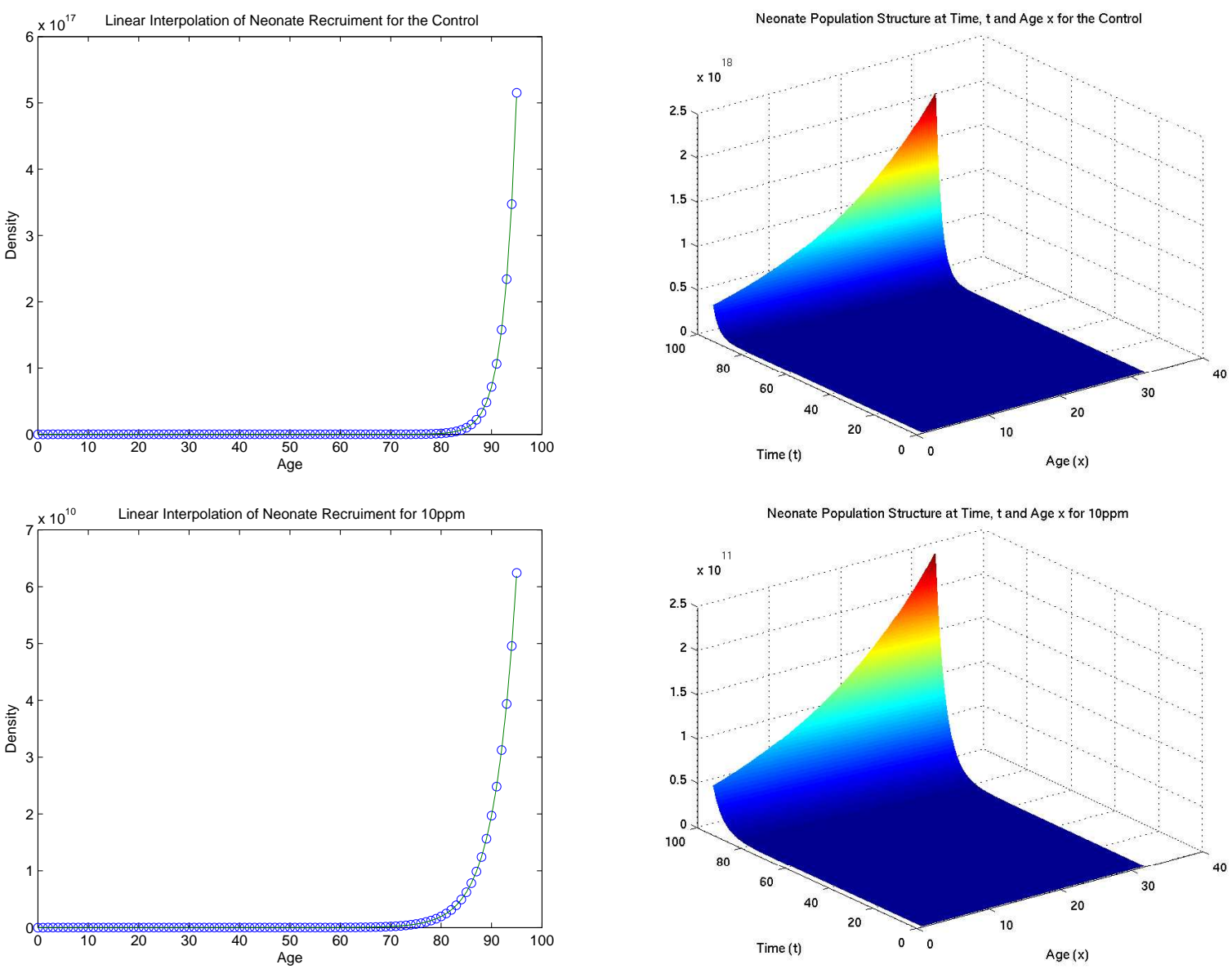

Figure 13: Neonate recruitment and population structure for the control and 10ppm

We notice that the maximum population level essentially corresponds with the maximum population level determined with the Leslie simulations. See Figures 4 and 5. These results lead us to 
accept that the SS model is producing believable results. There does not appear to be a substantial difference between SS and Leslie.

The recruitment function and SS results for neonate exposure at 20ppm are in Figure 14. As mentioned above in the section on Leslie results, as concentration level increases the population level ceases to be exponential. With Leslie for 20ppm, population growth was linear. We see that the SS simulation eventually produces linear growth, but begins with some fluctuations. These fluctuations were not seen in the Leslie simulations. We do however, still discover that the concentration increase has greatly affected the maximum population level.
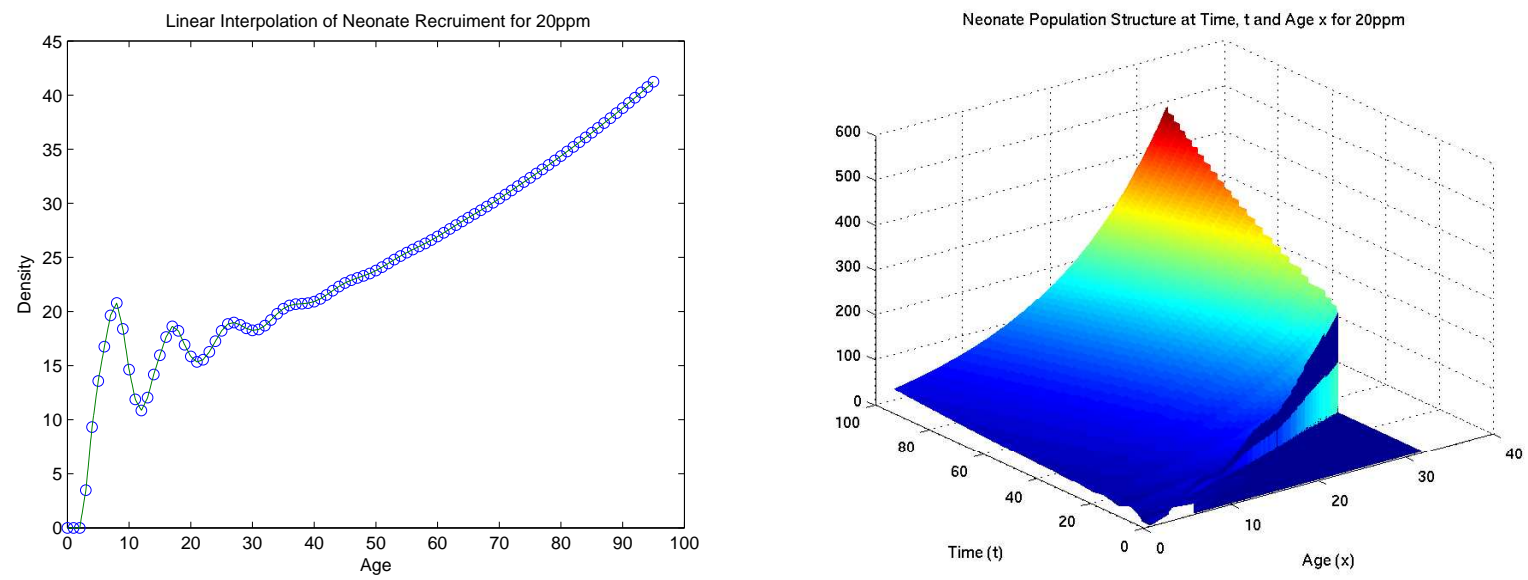

Figure 14: Neonate recruitment and population structure for 20ppm

With a neonate exposure of 40ppm (Figure 15), we see a low recruitment rate and a low maximum population rate. By time $t=30$, the population completely disappears. These results are similar to the Leslie results. The main difference between the two models, is that since the SS model is continuous, the population level does not remain at 100 individuals for all of day 1 . After a few time increments, the aphids have begun to drastically die out. In comparing the recruitment rate with the population structure, we see that there are a few individuals that survive the initial dose and age. The ones that survive, age a little, but eventually die out.

The results for neonate exposure of $60 \mathrm{ppm}$ are given in Figure 16. Again, we have hardly any recruitment and a very small population growth. The effect of Margosan-O is substantial. Although these results are comparable with the Leslie results, the Leslie model is not readily able to determine the small levels of aging. Because we are working with a continuous model when using SS, we are able to determine population dynamics not available with Leslie.

Results similar to those with an exposure level of 60ppm are seen with an exposure level of 80ppm. The results can be seen in Figure 17. Again we see that a small number of individuals survive using the SS model, whereas, there were no survivors with Leslie. However, we see that at 

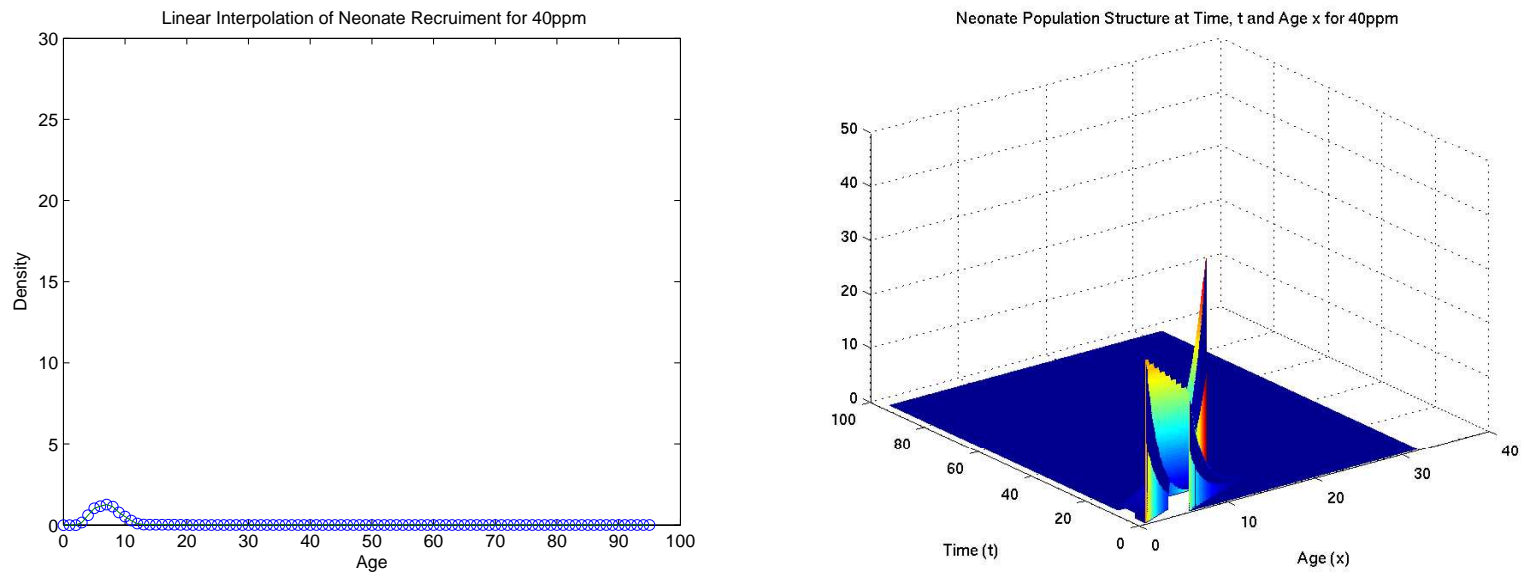

Figure 15: Neonate recruitment and population structure for 40ppm
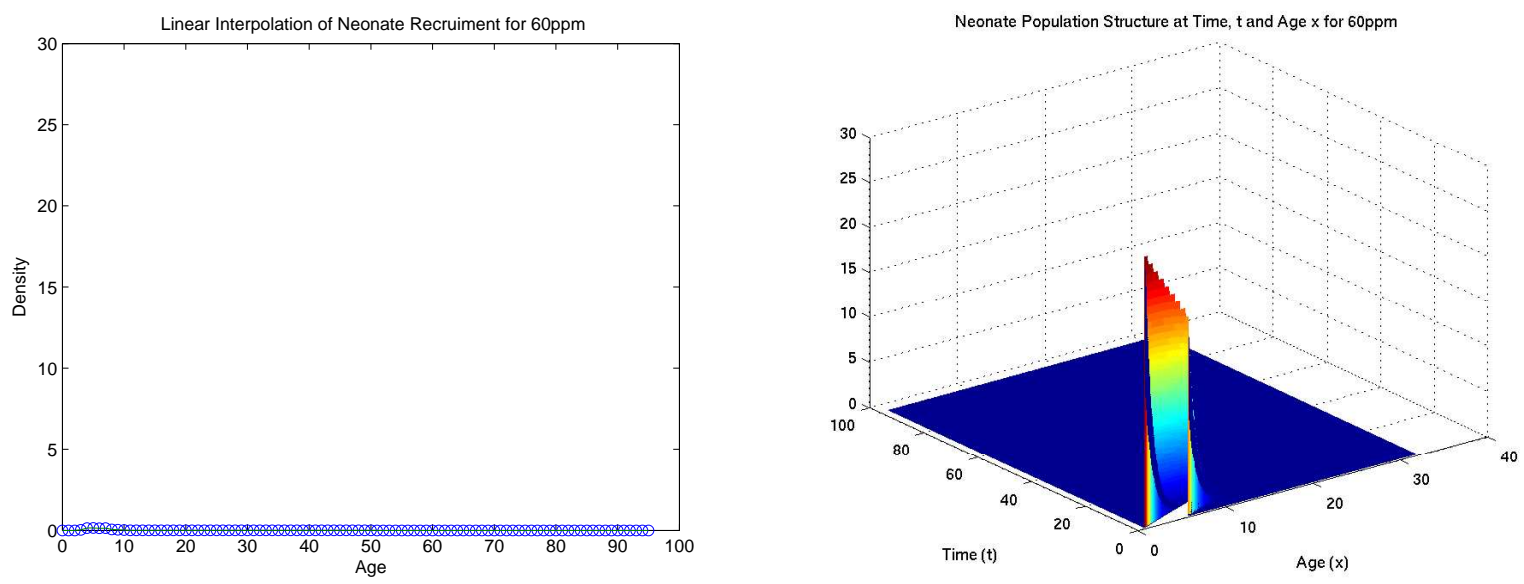

Figure 16: Neonate recruitment and population structure for $60 \mathrm{ppm}$

an exposure level of 80ppm, neonates cannot survive long. The population declines to zero. 

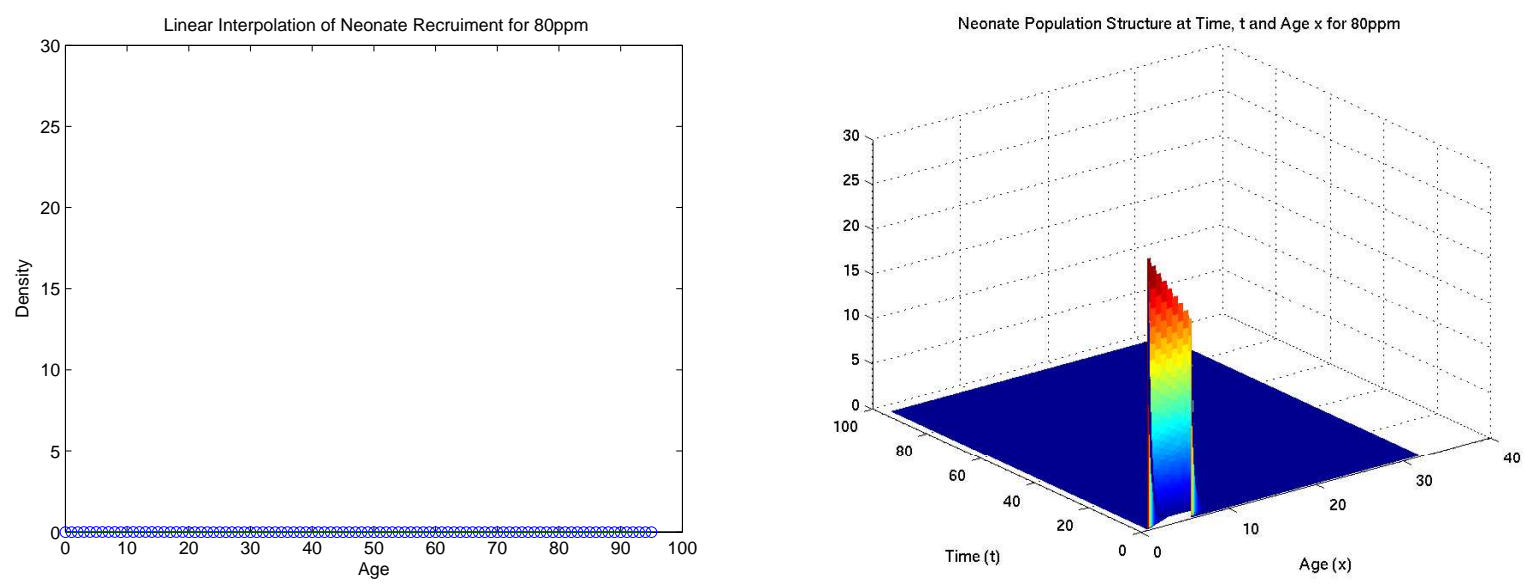

Figure 17: Neonate recruitment and population structure for 80ppm

\subsubsection{Adult Exposure}

We next summarize the Sinko Streifer adult exposure simulations. Results from adult exposure for the control and 10ppm concentrations can be seen in Figure 18.

We notice that the maximum population level essentially agrees with the maximum population level determined with the Leslie simulations. As we observed with the neonate exposure, these results lead us to believe that the SS model is producing reliable results from which conclusions can be drawn. There does not appear to be a substantial difference between the SS and the Leslie simulation results in these cases.

The results from 20ppm, 40ppm, 60ppm and 80ppm exposure can be seen in Figures 19 and 20. As with Leslie, we notice that an increase in the concentration level, decreases the maximum population levels, but not sufficiently enough to counteract exponential growth. 

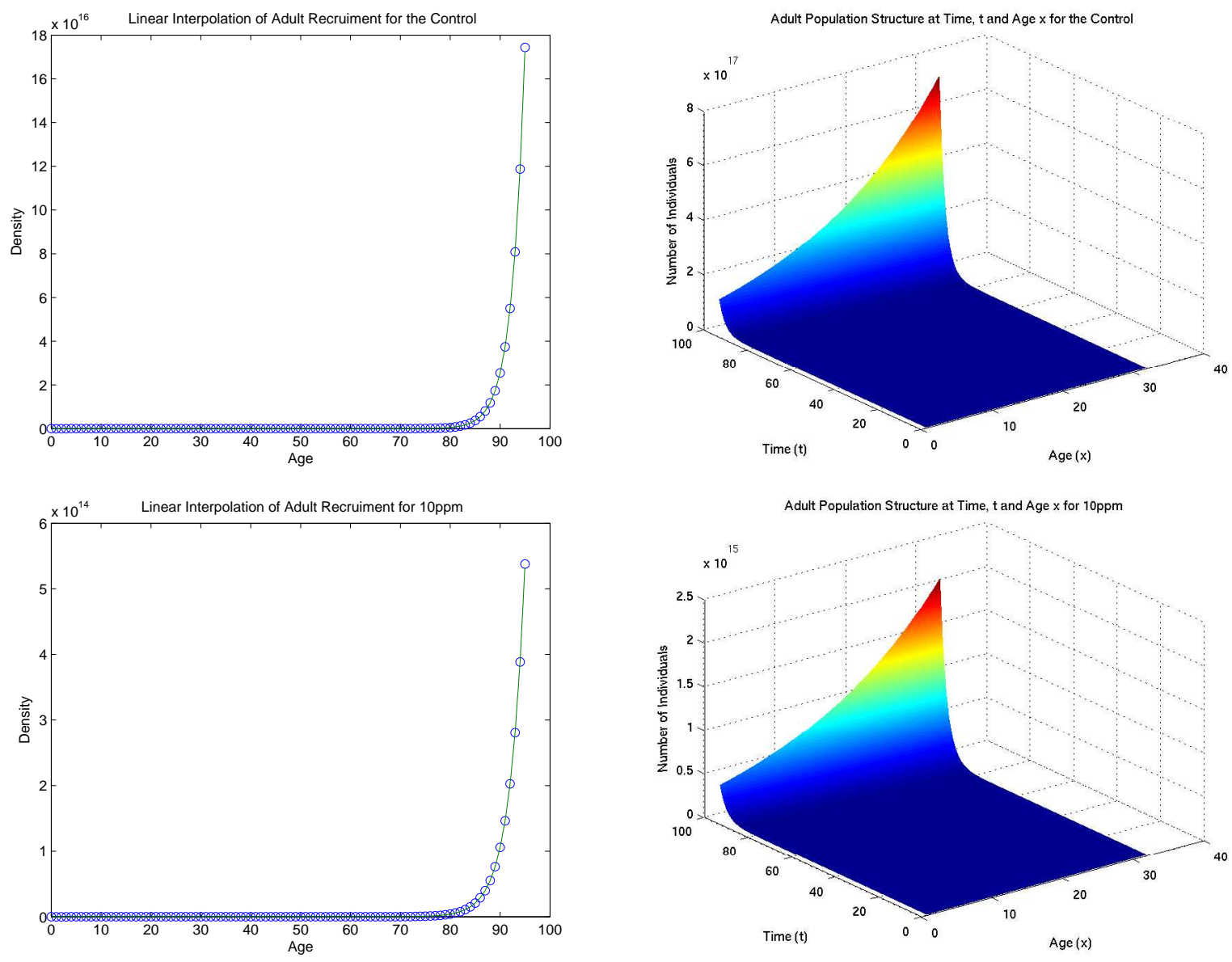

Figure 18: Adult recruitment and population structure for the control and $10 \mathrm{ppm}$ 

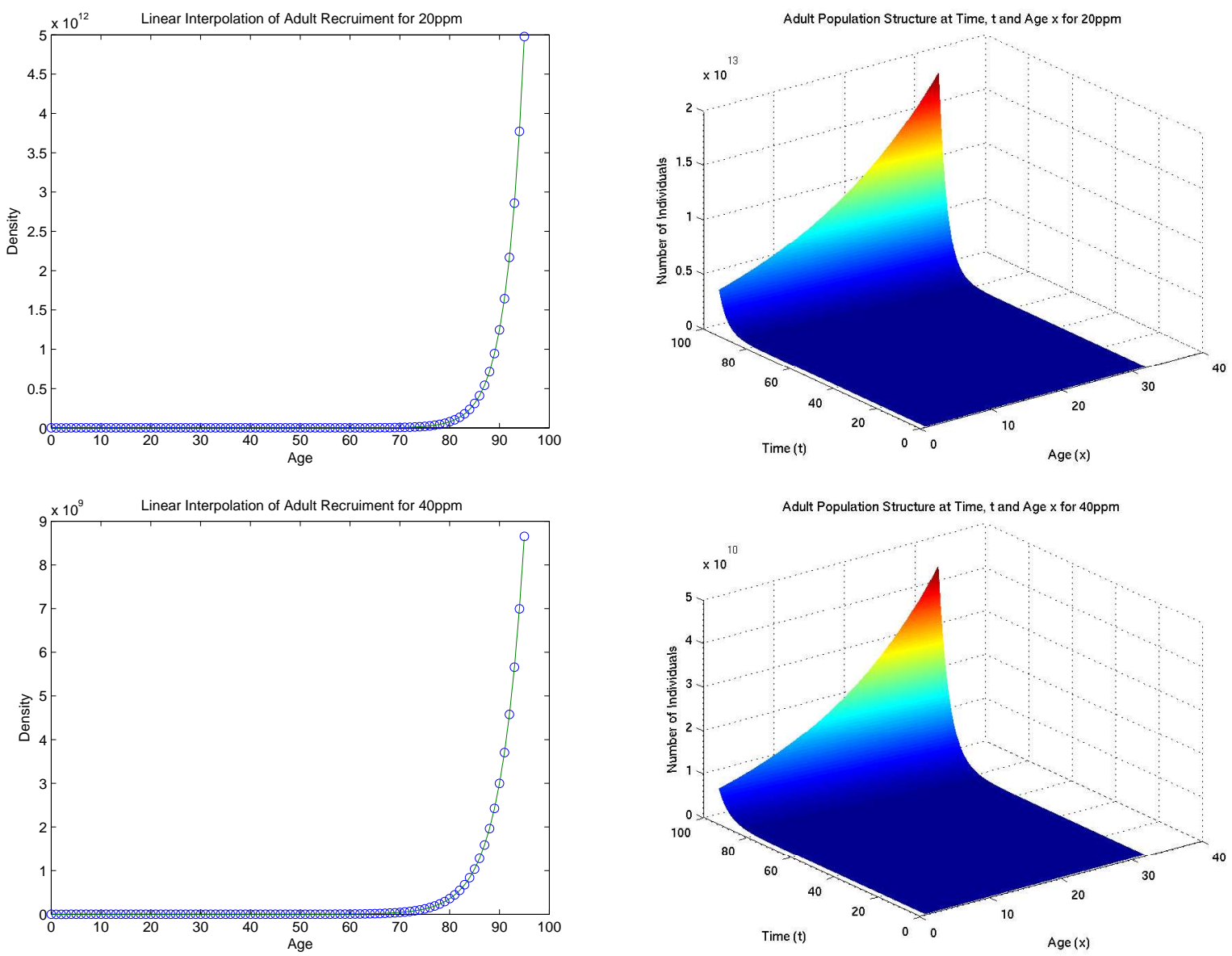

Figure 19: Adult recruitment and population structure for $20 \mathrm{ppm}$ and $40 \mathrm{ppm}$ 

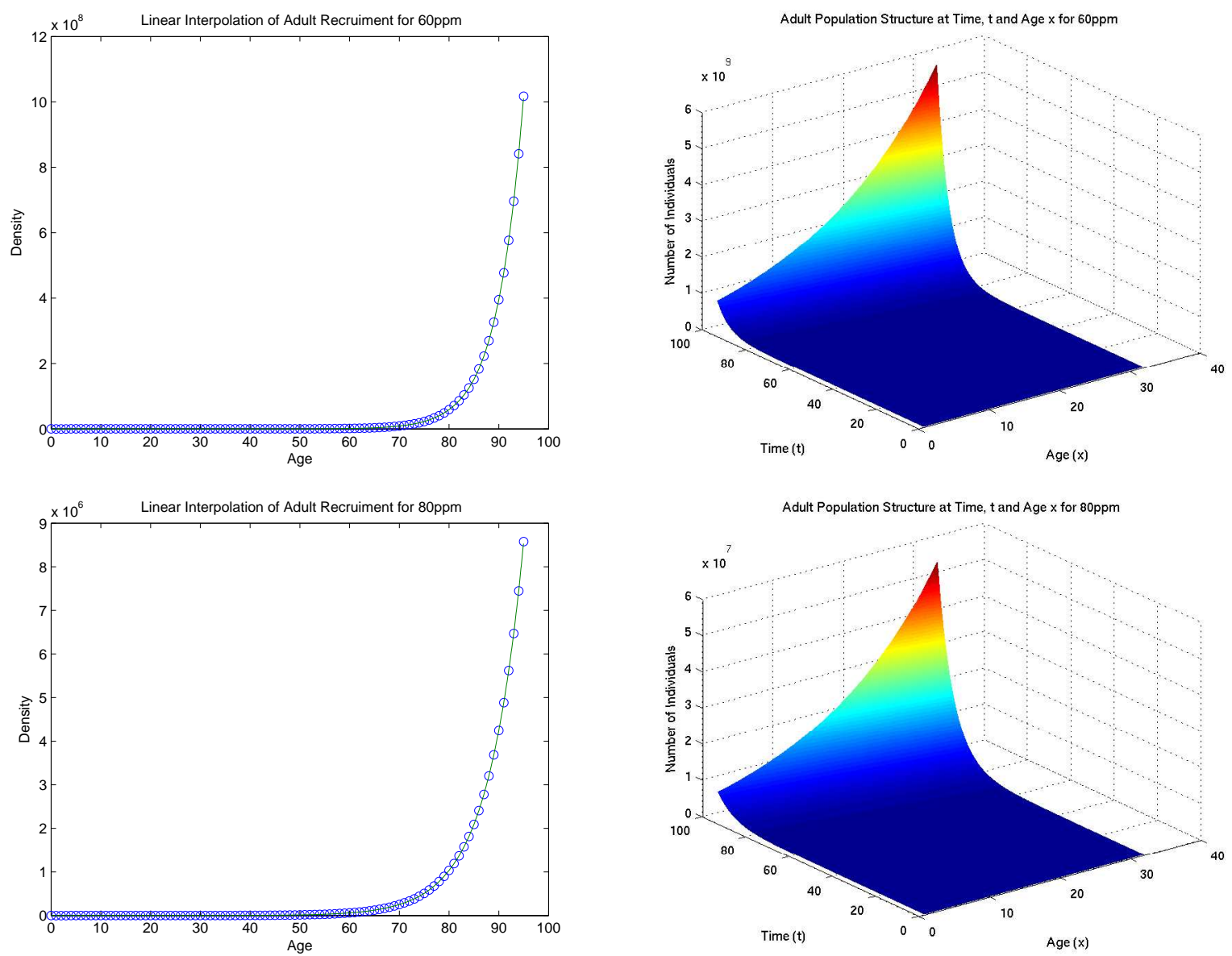

Figure 20: Adult recruitment and population structure for $60 \mathrm{ppm}$ and $80 \mathrm{ppm}$

The SS simulations for adult exposure appear to compare very closely to the Leslie simulations in the case of adults. On the other hand, the SS model is a better model when dealing with neonate exposure because sublethal effects can be seen with the continuous model. For example, in Figure 15 we are able to see fluctuations of population growth within the age classes. It is not possible to see these sublethal effects using the Leslie model. Also, Figures 16 and 17 depict the population actually recovering from the initial dosage. This recovery does not last long, but because Leslie is a discrete model, the potential recovery is not captured in our Leslie simulations. These observations lead us to suggest that SS is a potentially more useful model.

\subsubsection{Comments}

Since we have used results from the Leslie formulation to determine our parameters for Sinko Streifer, the values we obtain using the formulas from Table 1 are identical to those we calculated using Leslie. The only difference is the value of $r_{i}$, the instantaneous rate of increase. We calculate 
$r_{i}$ using the population totals for each day. Since we are dealing with a continuous model, $r_{i}$ has not been calculated. It may be possible to extrapolate the formula used to determine $r_{i}$ in the Leslie model to our SS model, but this has not been done. If we use each time increment as a "day" and compute $r_{i}$ using population totals from each time increment, we find that these $r_{i}$ values are much lower than they were with the Leslie simulations. They no longer are comparable to the $r_{m}$, intrinsic rate of increase.

\section{Parameter Estimation}

\subsection{Introduction}

Inverse problems in science and engineering are quite simply problems where the answer or solution is known, but not necessarily the question (i.e., dynamics). Applied mathematicians often "work backward" on problems with which they have experimental data, but not a mathematical model to describe the data. Their goal is to develop a model that best represents the experimental data while containing some measure of fidelity with the physical or biological phenomenon under investigation. We suspect that the SS model may provide us with a better representation of our data than did the Leslie model. Since we have experimental data with which to compare results, we performed an inverse problem to estimate parameters in the SS model. We used the resulting estimated parameter values to run simulations that we then compared to our experimental data. Throughout the presentation below, we discuss ramifications of using parameter estimates from Leslie in SS and also discuss limitations of the data with which we are working.

\subsection{Estimation of the Death Rate}

Recall the Sinko-Streifer model from equation (4). Both our initial condition driven solution, see equation (6), and our boundary condition driven solution, see equation (7), depend on values of $\mu$, our death rate. Death rates computed from our Leslie results (see Table 1 and Figure 9) were used to run all SS simulations in Section 3.1. Since our first goal is to compare the ability of the Leslie model to describe the experimental data with that of the SS model, we would like to estimate all parameters in SS independent of those in Leslie. In order to obtain a death rate specifically for SS, we estimate $\mu$ using an ordinary inverse least squares (OLS) technique. We wish to know what value(s) of $\mu$ will provide us with an accurate estimate of the population dynamics.

To estimate parameters with the OLS technique, we minimize the criterion

$$
J(\mu)=\sum_{i=1}^{N_{x}} \sum_{j=1}^{N_{t}}\left|U_{S S}\left(x_{i}, t_{j} ; \mu\right)-U_{i, j}^{d}\right|^{2},
$$

where $U_{S S}\left(x_{i}, t_{j} ; \mu\right)$ is the solution obtained by the method of characteristics and evaluated at age $x_{i}$ and time $t_{j}$ for given values of $\mu$, and $U_{i, j}^{d}$ is our experimental data. In order to compute the value 
of $\mu_{0}$ which minimizes the above cost function, we used the MATLAB routine fminsearch, which is a Nelder-Mead direct search method. Direct search methods $[\mathrm{Ke}]$ use values of $f$ taken from a set of sample points and use that information to continue the sampling. Direct search methods do not require approximate gradient information.

Before we can discuss results, we must discuss the experimental data with which we are working. Recall the experimental procedure detailed in Section 2.1. The experiment was designed to track one generation of aphids, namely the generation sprayed with pesticide. Table 2 contains an example of the type of data we have for all exposure levels for both neonates and adults. We know the amount being born into the population each day, but are not following the lives of these newborns. Thus, when we convert our experimental data into matrix form, we obtain information in the following form:

$$
\text { Age }\left[\begin{array}{ccccc}
\star & * & * & \ldots & * \\
0 & \star & 0 & \ldots & 0 \\
0 & 0 & \star & 0 \ldots & 0 \\
\vdots & 0 & \ldots & \ddots & 0 \\
0 & 0 & \ldots & \ldots & \star
\end{array}\right]
$$

with $\star$ representing the generation initially sprayed with pesticide and $*$ representing the individuals born into the population each day, which for our SS model represents our recruitment rate. Our SS simulations result in a "full" matrix, i.e., values of $u(x, t)$ for all $(x, t)$. Ideally, we would like to have data to compare with the full simulation matrix. However, this is not the case. We only have data along one characteristic (i.e, along $(x, t)=\left(x, x-x_{0}\right)$ ) with which to perform the inverse problem, namely the main diagonal that follows the initial population as it ages. Our results are based on measurements where we are only using one characteristic to estimate the optimal death rate $\mu_{0}$. We will discuss these and other implications in the following sections.

\subsection{Results-Estimating Constant Death Rates}

The results of the OLS problem for estimating $\mu$ for neonate exposure can be seen in Table 6 and in Figure 21. We note that all graphs for the inverse problem results represent calculations starting at $t_{0}=1$, corresponding to the data point $x_{0}$ for the population value at $t_{0}$ in the criterion from (10). We see that the estimated death rates are considerably lower than those calculated with the Leslie model. One of the purposes of performing this inverse problem is to give us a means with which to compare results from the Leslie and SS simulations. The values of the estimated death rates increase in a treatment concentration dependent manner similar to the increases seen with the Leslie model. However, the actual values are on a much smaller scale.

In studying the graphs in Figure 21, we see that the SS model with the optimal $\mu$ values 
Table 6: OLS results of estimation of $\mu$ for neonate exposure

\begin{tabular}{|c|c|c|c|}
\hline Concentration & $\mu$ from Leslie & OLS/SS $\mu$ & Estimated Cost \\
\hline Control & .0576 & .0128 & $1,110.2$ \\
\hline $10 \mathrm{ppm}$ & .0516 & .0277 & 571.5 \\
\hline $20 \mathrm{ppm}$ & .0810 & .0396 & 297.6 \\
\hline $40 \mathrm{ppm}$ & .3739 & .0951 & 114.6 \\
\hline $60 \mathrm{ppm}$ & .7174 & .2034 & 122.6 \\
\hline 80ppm & 1.7780 & .7936 & 3.3 \\
\hline
\end{tabular}

appears to better fit the data than the SS model with the $\mu$ obtained from Leslie. This is true for all exposure levels. Thus, in determining a death rate independent of Leslie, we obtain a death rate that better predicts our experimental data.

The results of the inverse problem for estimating $\mu$ for adult exposure are given in Table 7 and in Figure 21. These results are similar to those we observed for neonate exposure. Again, the values seem to be exposure treatment concentration dependent, but on a much smaller level than the death rates obtained from the Leslie model. Moreover, Figure 22 contains results similar to those for neonate exposure. The optimal $\mu$ appears to be a better estimation of death rate than the death rate corresponding to the Leslie formulation.

Table 7: OLS results of estimation of $\mu$ for adult exposure

\begin{tabular}{|c|c|c|c|}
\hline Concentration & $\mu$ from Leslie & OLS/SS $\mu$ & Estimated Cost \\
\hline Control & .0581 & .0173 & $\overline{766.3}$ \\
\hline $10 \mathrm{ppm}$ & .0540 & .0251 & 768.3 \\
\hline $20 \mathrm{ppm}$ & .0509 & .0337 & 587.4 \\
\hline 40ppm & .0597 & .0420 & 919.0 \\
\hline 60ppm & .0619 & .0417 & 737.0 \\
\hline 80ppm & .0643 & .0463 & 765.4 \\
\hline
\end{tabular}

One method of determining how well our model fits our experimental data is to study the cost associated with each of the inverse problems. The cost or error values for neonate exposure can be seen in Table 6 and values for adult exposure can be seen in Table 7. These cost values will provide us with a means of statistical comparison when we add more degrees of freedom to our model. It is of value to note that although the majority of the optimal rates yield models that only approximate the experimental data, the optimal death rate corresponding to a neonate exposure of $80 \mathrm{ppm}$ results in a cost of only three. This observation is apparent from Figure 21. The graphs of the experimental data and the SS simulation run with the optimal value for $80 \mathrm{ppm}$, lie almost on 

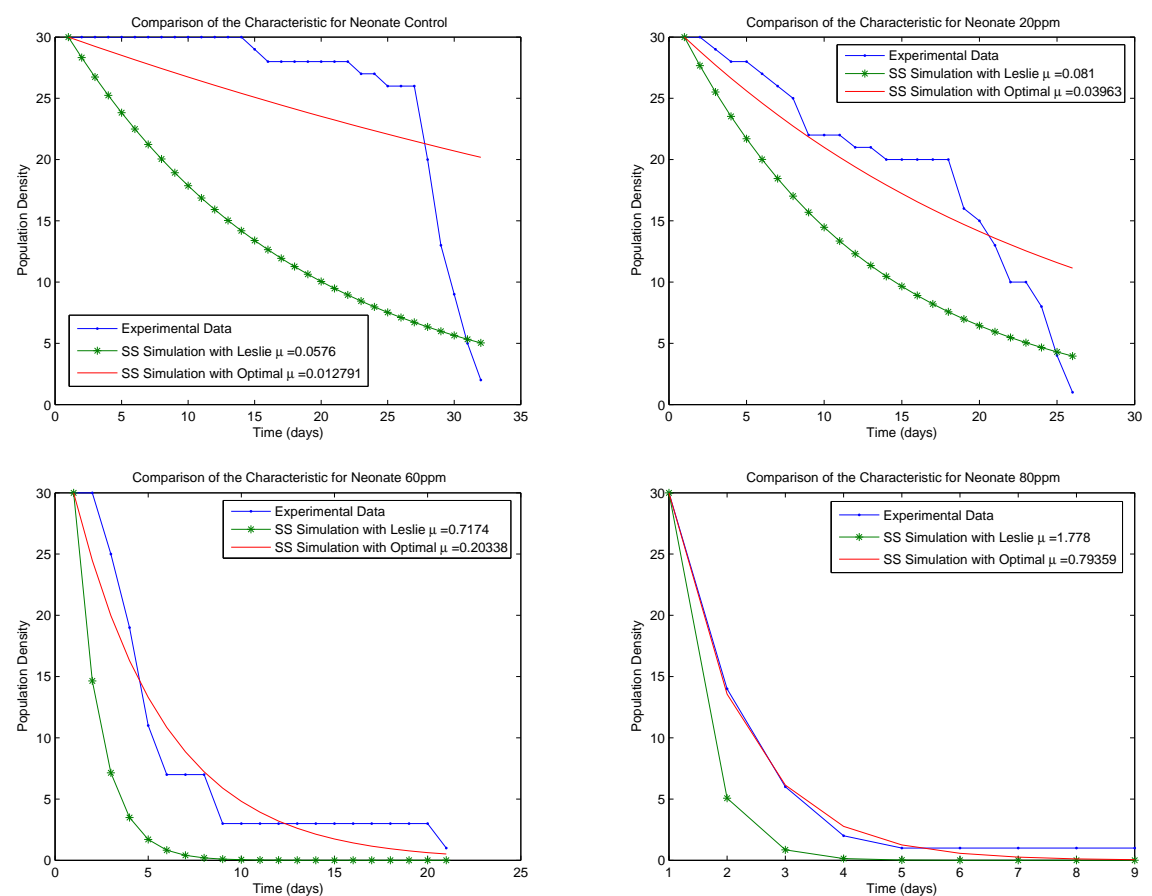

Figure 21: Comparisons of experimental data to SS simulations using Leslie $\mu$ and the optimal OLS $\mu$ for neonate exposure

top of each other. A possible explanation of this involves the fact that at an exposure of 80ppm, the experimental data contains deaths from day one to day two mirroring the mathematical effects of SS.

Again, comparing the Leslie obtained $\mu$ to our SS estimated $\mu$ using the SS model may not be a worthwhile comparison. There does not appear to be an acceptable correlation between the death rates obtained from Leslie and those obtained through our OLS problem. We believe that using the Leslie simulations to predict parameters for sublethal effects may not be as reliable as using SS.

To obtain a better understanding of our results, we decided to rerun the inverse problem using more "data". As stated before, the inverse problem was run using data from only one characteristic. That means that we determined the optimal OLS values for death rate based on only 9-32 data points, depending on exposure level and method. We hypothesize that given more experimental data, we would obtain more acceptable results. A second characteristic was added to our experimental data. We created this simulated data using SS simulations run with a systematic assortment of death rates that increase as concentration increases (See Tables 8 and 9). The first characteristic with nonzero densities resulting from the boundary condition driven solution was added to our experimental data to create a two characteristic data set. Results for the inverse 

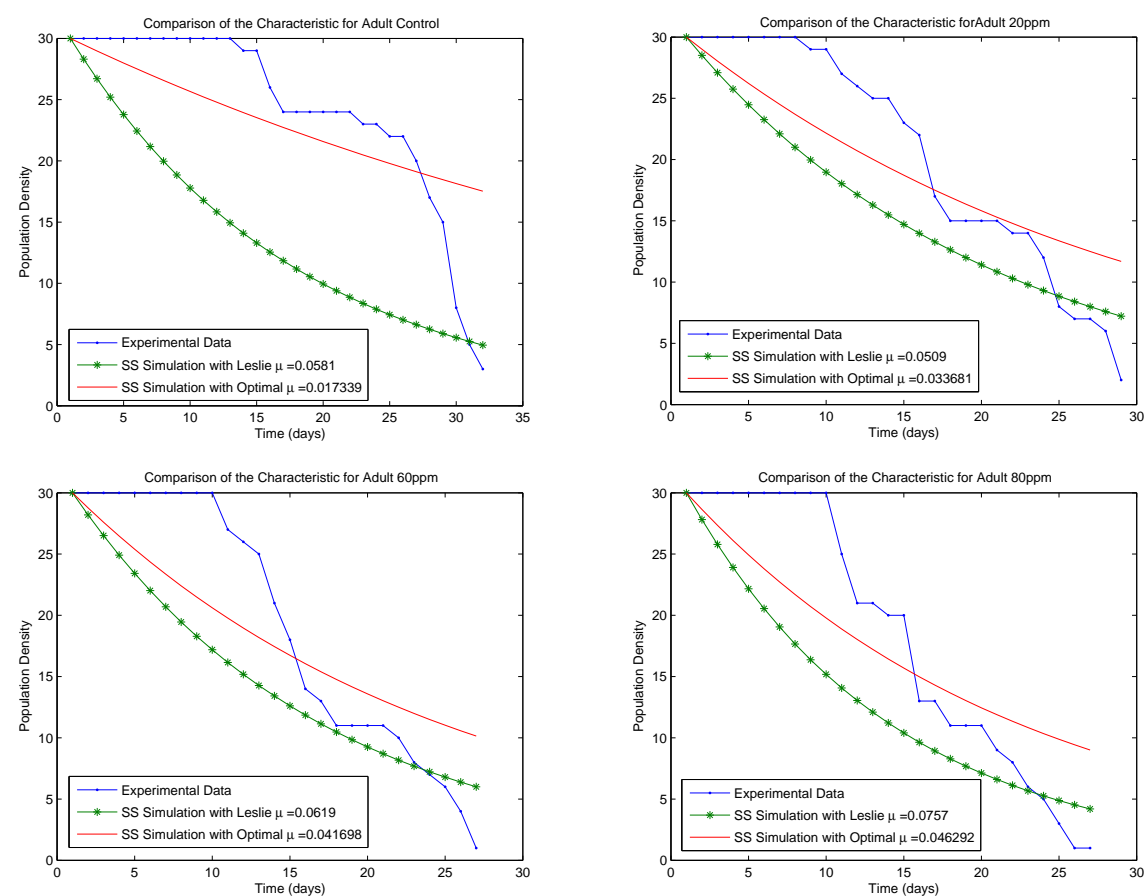

Figure 22: Comparisons of experimental data to SS simulations using Leslie $\mu$ and the optimal OLS $\mu$ for adult exposure

problem run with these two characteristics for neonate exposure can been found in Table 8, and results for adult exposure in Table 9.

Table 8: OLS results of estimation of $\mu$ for neonate exposure with two characteristics

\begin{tabular}{|c|c|c|c|}
\hline Concentration & \multicolumn{2}{|c|}{ Two Characteristics } & One Characteristic \\
\hline & Simulated Data $\mu$ & OLS/SS $\mu$ & OLS/SS $\mu$ \\
\hline \hline Control & .2 & .2 & .0128 \\
\hline $10 \mathrm{ppm}$ & .3 & .3 & .0277 \\
\hline $20 \mathrm{ppm}$ & .4 & .4 & .0396 \\
\hline $40 \mathrm{ppm}$ & .5 & .0951 & .0951 \\
\hline $60 \mathrm{ppm}$ & .6 & .2033 & .2034 \\
\hline $80 \mathrm{ppm}$ & .7 & .7937 & .7936 \\
\hline
\end{tabular}

These results suggest that when the second characteristic, dependent on recruitment, is added to our experimental data, we are sometimes able to match the death rates used to create the simulated data. For neonate exposure, we find a match in the optimized $\mu$ for the first three concentrations. The second three concentrations are so high that there are no aphids being born into the population, i.e., the recruitment is equal to zero. This causes the addition of another 
Table 9: OLS results of estimation of $\mu$ for adult exposure with two characteristics

\begin{tabular}{|c|c|c|c|}
\hline Concentration & \multicolumn{2}{|c|}{ Two Characteristics } & One Characteristic \\
\hline & Simulated Data $\mu$ & OLS/SS $\mu$ & OLS/SS $\mu$ \\
\hline \hline Control & .1 & .1 & .0173 \\
\hline $10 \mathrm{ppm}$ & .2 & .2 & .0251 \\
\hline $20 \mathrm{ppm}$ & .3 & .3 & .0337 \\
\hline $40 \mathrm{ppm}$ & .4 & .4 & .0420 \\
\hline $60 \mathrm{ppm}$ & .5 & .5 & .0417 \\
\hline $80 \mathrm{ppm}$ & .6 & .6 & .0463 \\
\hline
\end{tabular}

characteristic to be only the addition of zeros. Thus, our optimized values with two characteristics are almost precisely identical to those computed with only one characteristic. For adult exposure, we find that no matter what death rate we use to simulate the second characteristic, we are able to recover that same value with the inverse problem.

\subsubsection{Implications}

The above results serve to illustrate to us the importance of having more experimental data if one insists on models with constant death rate. Data along one characteristic does not appear to be enough to compute optimal constant values of the death rates unless the concentration of pesticide is so high that it immediately kills off the population. Through an analysis of the results obtained from our OLS problem, we see a sizable discrepancy between the constant death rate calculated from the Leslie simulation and the constant optimal death rate with SS; however neither model with these constant death rates yields a good fit to the experimental data.

We suspect that a main reason for the lack of good fit to our experimental data lies in the fact that when evaluating the experimental data there appears to be a time dependency on death rate. Consider equation (8). The initial condition driven solution incorporates the death rate into its first calculation, causing there to be deaths from day one to day two. However, our experimental data shows that for the neonate control, there are no deaths until day 15, and for the adult control, there are no deaths until day 13. This pattern follows for each concentration level and exposure method, except for neonate exposure of 80ppm. Again, for 80ppm, deaths do occur from day one to day two, allowing SS to more closely match the data. Recall that Leslie gave us a constant death rate, which we then used in our SS simulations. This suggests that perhaps death rate should not be considered as a constant, but rather should be time-dependent. This dependence would vary with exposure level and method.

Considering the nature of our experiments with the varying levels of application, we expect sublethal damage and hence a possible need for time dependent mortalities. Standard Leslie for- 
mulations do not readily permit us to consider death rate as a time dependent parameter. However time dependent death rates are easily accommodated by SS. Since SS is much more flexible, we believe it is more desirable to use for our attempts to model the experimental data.

\subsection{Time Dependent Death Rates}

In general, a species' death rate may change as individuals move through different life stages. Thus, even for the control experiments, there is little reason for us to believe that the aphids have a constant death rate. In addition to aging, they are also being exposed to pesticide. We should expect to see better results if we allow $\mu$ to be time dependent, especially if we believe sublethal damage occurs during pesticide exposure. Therefore we next consider simulations with $\mu=\mu(t)$ in the SS model.

We take $\mu$ as a time dependent, piecewise constant variable by creating a parameterization of the time dependent function given by

$$
\mu(t) \approx \mu^{M}(t)=\sum_{j=1}^{M} a_{j} \chi_{j}^{M}(t),
$$

where

$$
\chi_{j}^{M}= \begin{cases}1 & {\left[t_{j-1}^{M}, t_{j}^{M}\right)} \\ 0 & \text { elsewhere }\end{cases}
$$

with time increments $t_{j}^{M}$. We are thus seeking to estimate the $M$ nodal values $\left\{a_{j}\right\}_{j=1}^{M}$ for $\mu^{M}(t)$ that provide a good fit to the experimental data.

Since our death rate is no longer constant, we must return to equation (6) to derive a new SS solution. However, our observations only contain data along one characteristic, and thus it is only necessary for us to derive a new solution for the initial condition driven solution $(\mathrm{t} \leq \mathrm{G}(\mathrm{x}))$. Along the characteristic $X(0 ; x, t)$ we obtain

$$
\begin{aligned}
u^{M}(x, t) & =\Phi(X(0 ; x, t)) e^{-\int_{0}^{t}\left[g_{x}(\xi, X(\xi ; x, t))+\mu^{M}(\xi, X(\xi ; x, t))\right] d \xi} \\
& =\Phi(x-t) e^{\int_{0}^{t} \mu^{M}(\xi) d \xi} \\
& =\Phi(x-t) e^{-\int_{0}^{t} \sum_{j=1}^{M} a_{j} \chi_{j}^{M}(\xi) d \xi} \\
& =\Phi(x-t) e^{-\sum_{j=1}^{M} a_{j} \int_{0}^{t} \chi_{j}^{M}(\xi) d \xi}
\end{aligned}
$$

Thus our SS model with time dependent death rate with, for example, $M=4$ is given by

$$
u^{M}(x, t)=\Phi(x-t) e^{-\int_{0}^{t} \mu^{M}(\xi) d \xi}
$$


with

$$
e^{\int_{0}^{t} \mu^{M}(\xi) d \xi}= \begin{cases}e^{-a_{1} t} & {\left[0, t_{1}^{M}\right)} \\ e^{\left(a_{2}-a_{1}\right) t_{1}^{M}} e^{-a_{2} t} & {\left[t_{1}^{M}, t_{2}^{M}\right)} \\ e^{\left(a_{3}-a_{2}\right) t_{2}^{M}} e^{\left(a_{2}-a_{1}\right) t_{1}^{M}} e^{-a_{3} t} & {\left[t_{2}^{M}, t_{3}^{M}\right)} \\ e^{\left(a_{4}-a_{3}\right) t_{3}^{M}} e^{\left(a_{3}-a_{2}\right) t_{2}^{M}} e^{\left(a_{2}-a_{1}\right) t_{1}^{M}} e^{-a_{4} t} & {\left[t_{3}^{M}, t\right)}\end{cases}
$$

Table 10: OLS results of estimation of $\mu(t)$ for adult exposure

\begin{tabular}{|c|c|c|c|c|}
\hline & $\begin{array}{c}\text { Estimated } \mu \\
\text { Constant }\end{array}$ & $\begin{array}{c}\text { Estimated } \mu \\
\mathbf{2} \text { Nodes }\end{array}$ & $\begin{array}{c}\text { Estimated } \mu \\
\text { 3 Nodes }\end{array}$ & $\begin{array}{c}\text { Estimated } \mu \\
\text { 4 Nodes }\end{array}$ \\
\hline \hline Control & .0173 & {$[.0027, .0599]$} & {$[.0013, .0175, .1004]$} & {$[0, .0194, .0106, .1755]$} \\
\hline 10ppm & .0251 & {$[.0082, .0802]$} & {$[.0081, .0215, .1505]$} & {$[.0019, .0291, .0250, .2448]$} \\
\hline $\mathbf{2 0 p p m}$ & .0337 & {$[.0134, .1118]$} & {$[.0031, .0667, .1211]$} & {$[0, .0437, .0752, .1735]$} \\
\hline $\mathbf{4 0 p p m}$ & .0420 & {$[.0172, .1651]$} & {$[.0009, .0999, .1760]$} & {$[0, .0579, .1178, .2422]$} \\
\hline $\mathbf{6 0 p p m}$ & .0417 & {$[.0159, .1600]$} & {$[0, .1035, .1256]$} & {$[0, .0520, .1313, .1472]$} \\
\hline $\mathbf{8 0 p p m}$ & .0463 & {$[.0192, .1795]$} & {$[.0024, .1086, .1819]$} & {$[0, .0649, .1249, .2666]$} \\
\hline
\end{tabular}

\begin{tabular}{|c|c|c|c|c|c|c|}
\hline & \multicolumn{3}{|c|}{$\begin{array}{l}\text { Estimated } \mu \\
5 \text { Nodes }\end{array}$} & \multicolumn{3}{|c|}{$\begin{array}{l}\text { Estimated } \mu \\
6 \text { Nodes }\end{array}$} \\
\hline Control & \multicolumn{3}{|c|}{$[0, .0033, .0382, .0004, .2826]$} & \multicolumn{3}{|c|}{$[0,0, .0314,0, .1059, .0573]$} \\
\hline 10ppm & \multicolumn{3}{|c|}{$[0, .0263, .0189, .0532, .3381]$} & \multicolumn{3}{|c|}{$[0, .0163, .0337,0, .1597, .7275]$} \\
\hline 20ppm & \multicolumn{3}{|c|}{$[0, .0189, .0883, .0473, .2667]$} & \multicolumn{3}{|c|}{$[0, .0105, .0584, .0741, .1180, .2575]$} \\
\hline 40ppm & \multicolumn{3}{|c|}{$[0, .0194, .1303, .0917, .3451]$} & \multicolumn{3}{|c|}{$[0, .0012, .1058, .0870, .1840, .0124]$} \\
\hline \multirow{2}{*}{ 80ppm } & \multirow{2}{*}{\multicolumn{3}{|c|}{$\begin{array}{l}{[0, .0108, .1529, .0441, .2919]} \\
{[0, .0310, .1260, .0909, .4438]}\end{array}$}} & \multicolumn{3}{|c|}{$[0,0, .0919, .1305, .1121, .3201]$} \\
\hline & & & & {$[0, .0066, .108$} & $.1035, .185$ & $.2563]$ \\
\hline & $\begin{array}{c}\text { Cost } \\
\text { Constant }\end{array}$ & $\begin{array}{c}\text { Cost } \\
2 \text { Nodes }\end{array}$ & $\begin{array}{l}\text { Cost } \\
3 \text { Nodes }\end{array}$ & $\begin{array}{l}\text { Cost } \\
4 \text { Nodes }\end{array}$ & $\begin{array}{l}\text { Cost } \\
5 \text { Nodes }\end{array}$ & $\begin{array}{c}\text { Cost } \\
6 \text { Nodes }\end{array}$ \\
\hline Control & 766.3 & 307.0 & 185.3 & 95.6 & 33.3 & 163.2035 \\
\hline 10ppm & 768.3 & 245.2 & 92.4 & 50.9 & 39.9 & 67.7637 \\
\hline 20ppm & 587.4 & 105.5 & 55.2 & 40.7 & 27.9 & 53.1561 \\
\hline 40ppm & 919.0 & 217.3 & 50.2 & 79.1 & 39.1 & 49.0498 \\
\hline 60ppm & 737.0 & 163.3 & 45.5 & 72.6 & 17.3 & 41.5377 \\
\hline 80ppm & 765.4 & 162.1 & 43.0 & 49.5 & 50.9 & 42.0580 \\
\hline
\end{tabular}

To solve the inverse problem using our new model from equation (12), we again used the MATLAB routine fminsearch. For adult exposure, our MATLAB program provides the results seen in Table 10. Theoretical arguments related to increased degrees of freedom guarantee that we should get at least as good a value of the cost function each time we add parameters through refinements to our original parameterization (see $[\mathrm{BK}]$ ). With the statistical tests we employ here, we can most readily compare results for refinements of our parameterization. Thus we can look at comparisons of one (i.e., $\mu$ constant) versus any number of additional parameters, two versus four 
parameters and three versus six parameters.

In considering the adult exposure comparisons mentioned above, for a number of cases our initial run of the algorithm resulted in cost function values that were contrary to the theory mentioned above (i.e., additional "nested" parameters should result in decreased cost). We decreased the convergence parameter tolerance, and then obtained acceptable cost function values for all nodal values and all exposure levels. We chose Nelder-Mead because of its simplicity and good track record. It appears to have been an acceptable choice.

Table 11: OLS results of estimation of $\mu(t)$ for neonate exposure

\begin{tabular}{|c|c|c|c|c|}
\hline & $\begin{array}{c}\text { Estimated } \mu \\
\text { Constant }\end{array}$ & $\begin{array}{c}\text { Estimated } \mu \\
\mathbf{2} \text { Nodes }\end{array}$ & $\begin{array}{c}\text { Estimated } \mu \\
\mathbf{3} \text { Nodes }\end{array}$ & $\begin{array}{c}\text { Estimated } \mu \\
\mathbf{4} \text { Nodes }\end{array}$ \\
\hline \hline Control & .0128 & {$[0, .0501]$} & {$[.0013,0, .1112]$} & {$[0, .0070,0, .1972]$} \\
\hline $\mathbf{1 0 p p m}$ & .0277 & {$[.0098, .0870]$} & {$[.0134, .0181, .1658]$} & {$[.0180, .0010, .0613, .2031]$} \\
\hline $\mathbf{2 0 p p m}$ & .0396 & {$[.0253, .0897]$} & {$[.0310, .0204, .1863]$} & {$[.0270, .0328, .0345, .2799]$} \\
\hline $\mathbf{4 0 p p m}$ & .0951 & {$[.1057, .0385]$} & {$[.1087, .0729, .0406]$} & {$[.0956, .1350,0, .1010]$} \\
\hline $\mathbf{6 0 p p m}$ & .2034 & {$[.2127, .0300]$} & {$[.2123, .1595,0]$} & {$[.1987, .2693,0, .0394]$} \\
\hline $\mathbf{8 0 p p m}$ & .7936 & {$[.7959,0]$} & {$[.8059, .4217,0]$} & {$[.7895, .8492,0,0]$} \\
\hline
\end{tabular}

\begin{tabular}{|c|c|c|}
\hline & $\begin{array}{c}\text { Estimated } \mu \\
\text { 5 Nodes }\end{array}$ & $\begin{array}{c}\text { Estimated } \mu \\
\mathbf{6} \text { Nodes }\end{array}$ \\
\hline \hline Control & {$[0, .0018, .0120,0, .3307]$} & {$[0, .0003, .0040,0, .1098, .0768]$} \\
\hline $\mathbf{1 0 p p m}$ & {$[.0186, .0065, .0124, .1080, .2184]$} & {$[.0196, .0089, .0037, .0411, .1550, .0805]$} \\
\hline $\mathbf{2 0 p p m}$ & {$[.0203, .0515,0, .0978, .3204]$} & {$[.0165, .0526, .0223, .0030, .1953, .0138]$} \\
\hline $\mathbf{4 0 p p m}$ & {$[.0769, .1888, .0151,0, .1797]$} & {$[.0596, .2146, .0502,0, .0722, .0523]$} \\
\hline $\mathbf{6 0 p p m}$ & {$[.1754, .3769,0,0, .1369]$} & {$[.1429, .4582, .0528,0, .0495, .0399]$} \\
\hline $\mathbf{8 0 p p m}$ & {$[.7646,1.1222, .0031,0,0]$} & {$[.7568,1.0099, .5365,0,0, .2835]$} \\
\hline
\end{tabular}

\begin{tabular}{|c|c|c|c|c|c|c|}
\hline & $\begin{array}{c}\text { Cost } \\
\text { Constant }\end{array}$ & $\begin{array}{c}\text { Cost } \\
\text { 2 Nodes }\end{array}$ & $\begin{array}{c}\text { Cost } \\
\text { 3 Nodes }\end{array}$ & $\begin{array}{c}\text { Cost } \\
\text { 4 Nodes }\end{array}$ & $\begin{array}{c}\text { Cost } \\
\text { 5 Nodes }\end{array}$ & $\begin{array}{c}\text { Cost } \\
\text { 6 Nodes }\end{array}$ \\
\hline \hline Control & 1110.2 & 557.7 & 288.9 & 128.5 & 27.3 & 287.6750 \\
\hline $\mathbf{1 0 p p m}$ & 571.5 & 113.8 & 39.9 & 32.4 & 17.0 & 28.0543 \\
\hline $\mathbf{2 0 p p m}$ & 297.6 & 131.3 & 37.7 & 37.6 & 23.1 & 26.6412 \\
\hline $\mathbf{4 0 p p m}$ & 114.6 & 80.3 & 86.8 & 66.2 & 40.6 & 31.4375 \\
\hline $\mathbf{6 0 p p m}$ & 122.6 & 99.8 & 107.0 & 93.6 & 74.1 & 54.3485 \\
\hline $\mathbf{8 0 p p m}$ & 3.3 & .967 & 1.585 & .917 & .005 & .1210 \\
\hline
\end{tabular}

Table 11 contains the MATLAB program results for neonate exposure. In this case, computations for comparisons of our simulations with neonate exposure also produced cost function values corresponding to theoretical predictions for all exposure levels and all nodal values. 


\subsection{Comparisons}

We next compare our simulations and results for different types of exposure. Simulations run using Leslie's constant $\mu$, SS's optimal constant $\mu$ and SS's time dependent $\mu^{M}(t)$ with $M=2$ nodal values are compared with our experimental data in Figures 23 (adult exposure) and 24 (neonate exposure), with $M=3$ nodal values in Figures 25 (adult exposure) and 26 (neonate exposure), with $M=4$ nodal values in Figures 27 (adult exposure) and 28 (neonate exposure), with $M=5$ nodal values in Figures 29 (adult exposure) and 30 (neonate exposure) and with $M=6$ nodal values in Figures 31 (adult exposure) and 32 (neonate exposure).
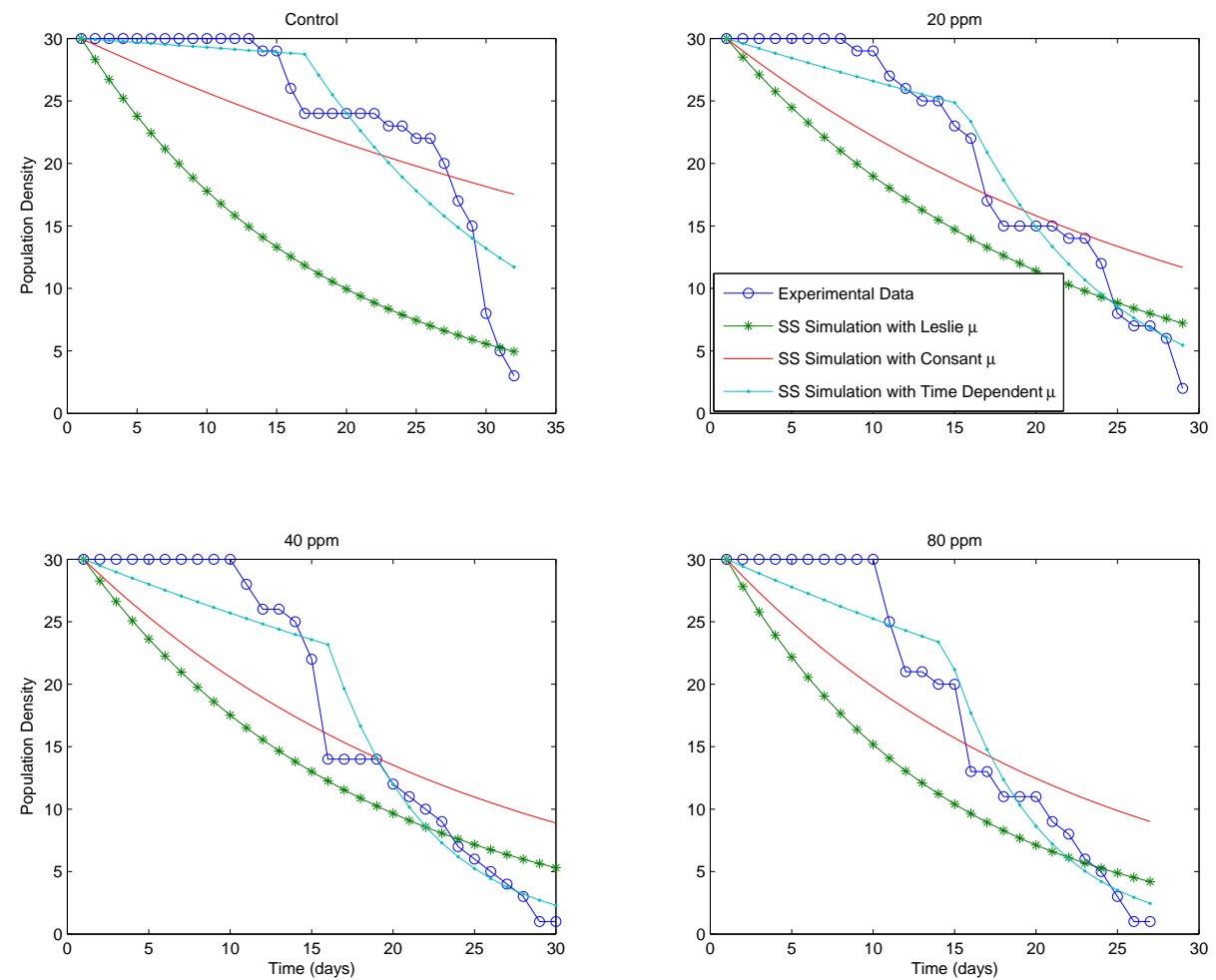

Figure 23: Comparisons of experimental data to SS simulations using Leslie $\mu$, the optimal constant $\mu$ and the optimal $\mu^{M}(t)$ with 2 nodes obtained for adult exposure

For adult exposure with two nodal values (Figure 23), the SS simulation using $\mu^{M}(t)$ appears to better fit the experimental data than the constant $\mu$, however, we do not appear to have found a death rate that is a close match of the experimental data. This is true for all exposure levels. Neonate exposure with $M=2$ for $\mu(t)$ (Figure 24) also appears to better fit the experimental data. However as exposure level increases, we see that our time dependent $\mu^{M}(t)$ produces similar results to the SS optimal constant $\mu$. 

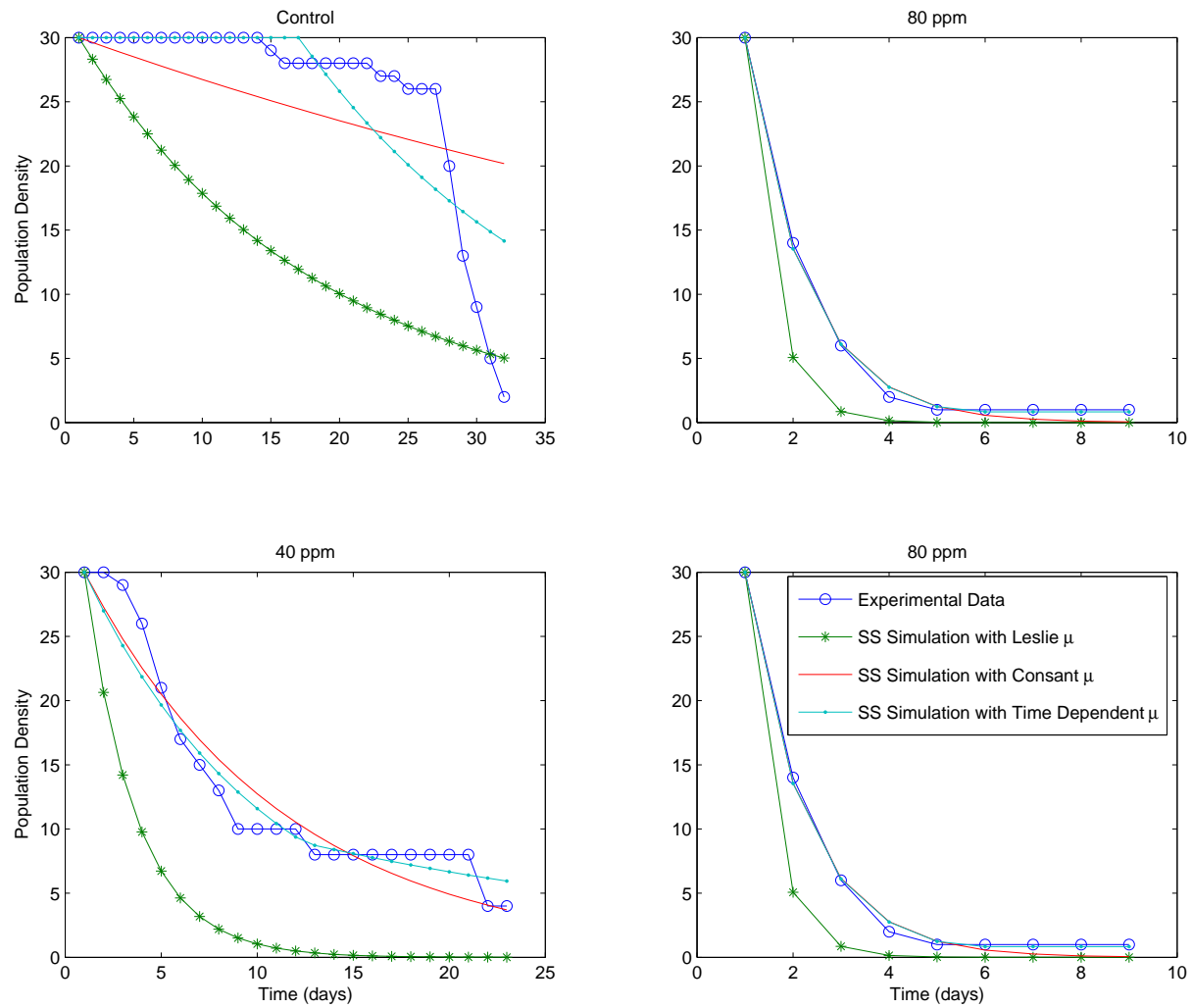

Figure 24: Comparisons of experimental data to SS simulations using Leslie $\mu$, the optimal constant $\mu$ and the optimal $\mu^{M}(t)$ with 2 nodes obtained for neonate exposure 

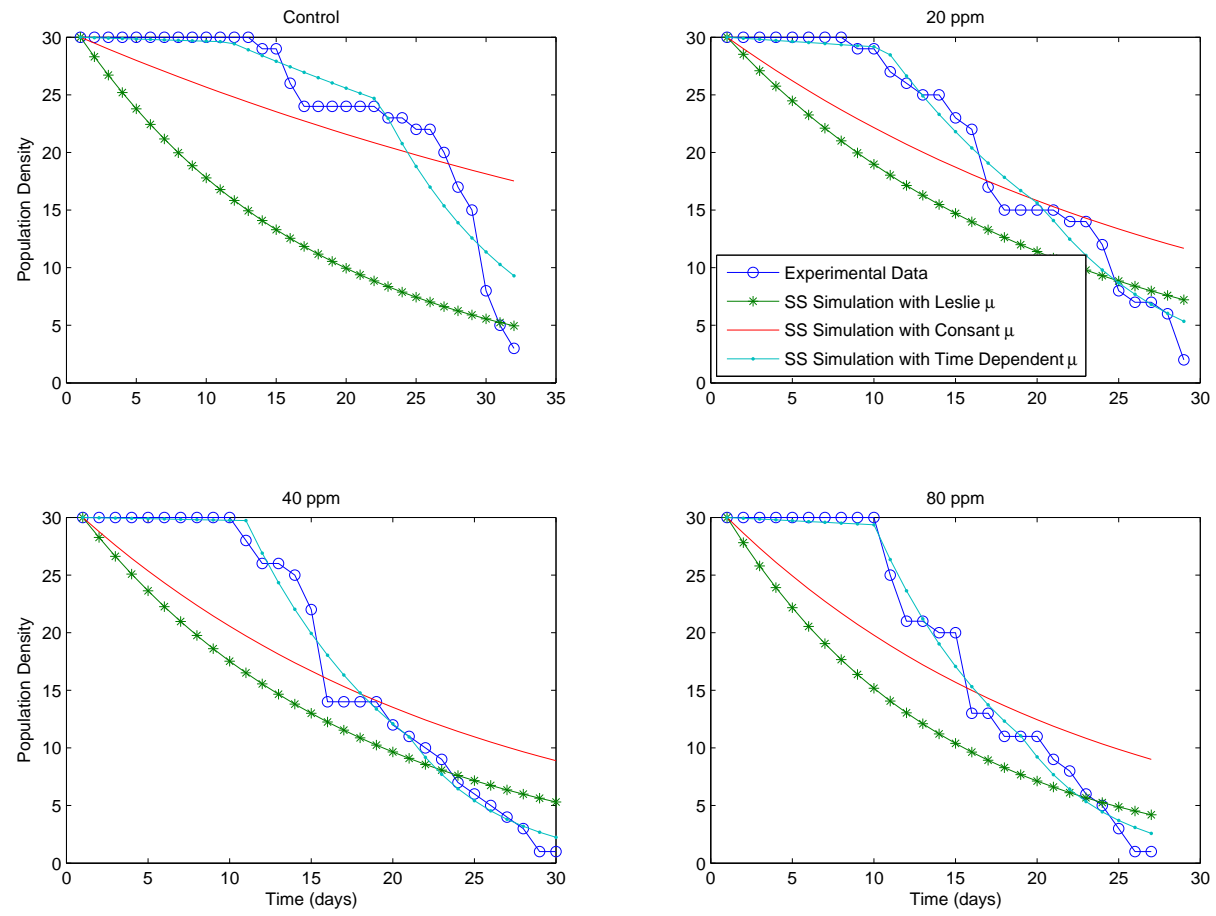

Figure 25: Comparisons of experimental data to SS simulations using Leslie $\mu$, the optimal constant $\mu$ and the optimal $\mu^{M}(t)$ with 3 nodes obtained for adult exposure

With the increase to three nodal values in $\mu^{M}(t)$, the results for adult exposure (Figure 25) again appear to better match the experimental data. As concentration level increases, we seem to obtain closer matches. For neonate exposure with $M=3$ for $\mu^{M}(t)$ (Figure 26), we see that for low levels of exposure three nodal values for $\mu^{M}(t)$ seem to better fit the data, but as exposure level increases, results with $\mu^{M}(t)$ are very close to those with a constant $\mu$. 

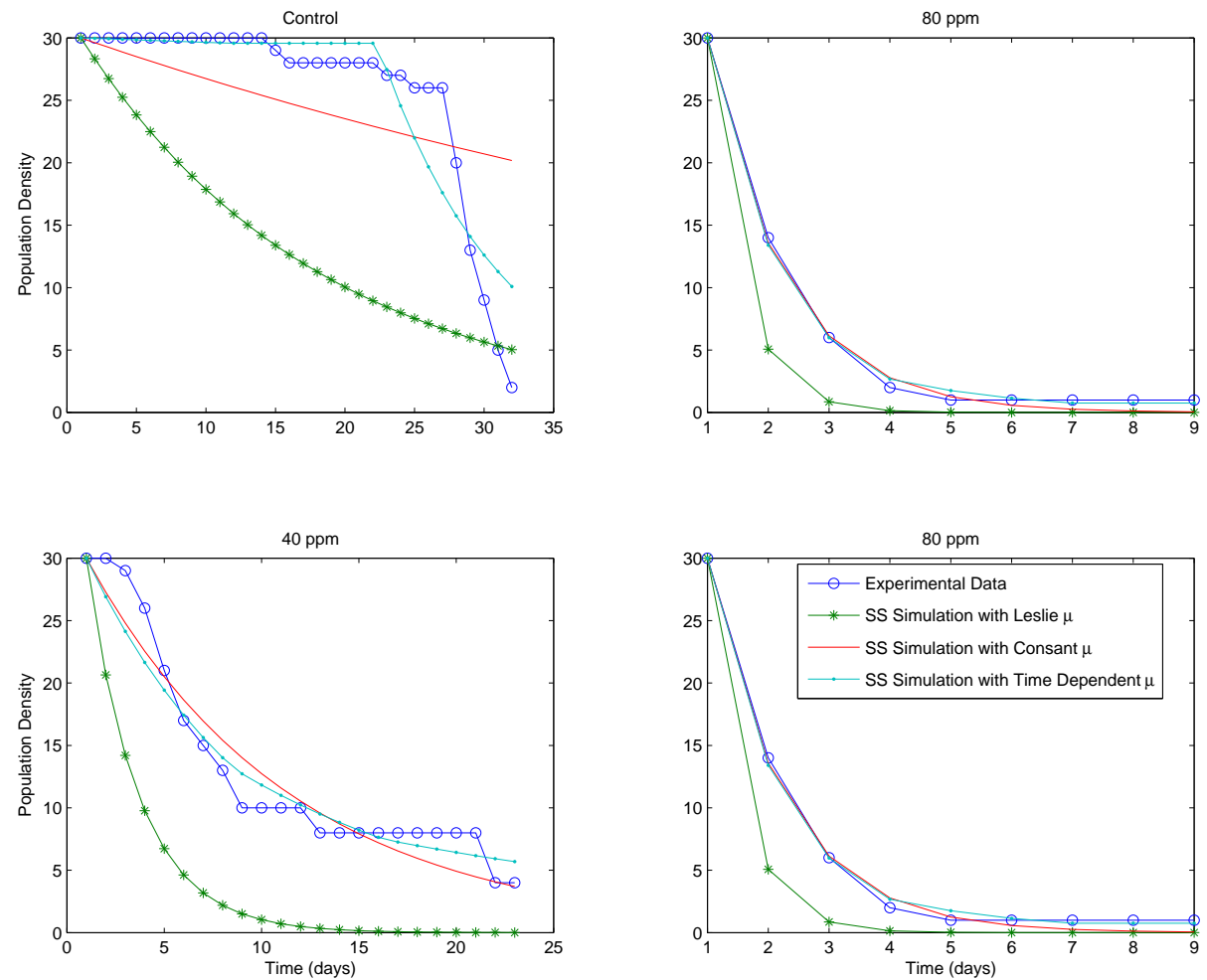

Figure 26: Comparisons of experimental data to SS simulations using Leslie $\mu$, the optimal constant $\mu$ and the optimal $\mu^{M}(t)$ with 3 nodes obtained for neonate exposure 

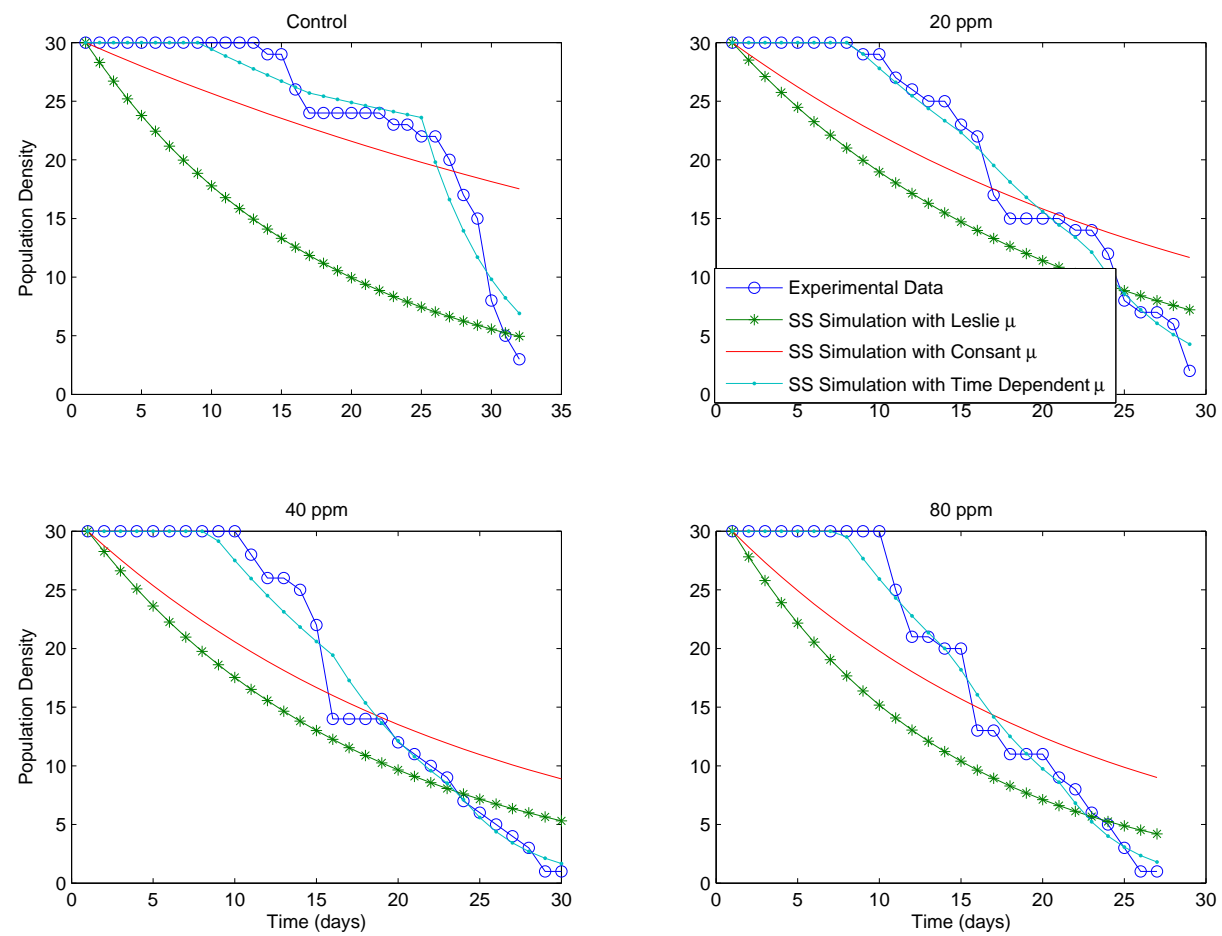

Figure 27: Comparisons of experimental data to SS simulations using Leslie $\mu$, the optimal constant $\mu$ and the optimal $\mu^{M}(t)$ with 4 nodes obtained for adult exposure

For adult exposure with four nodal values of $\mu^{M}(t)$ (Figure 27), we see that the simulation trajectories with $\mu^{M}(t)$ appear to closely match our experimental data. We are obtaining a better approximation to our experimental data each time we increase our nodal values for $\mu^{M}(t)$. The time dependent death rate is able to more closely approximate our experimental data than do solutions with the constant death rates from Leslie or solutions from our initial optimization of SS with constant mortality.

With neonate exposure $M=4$ (Figure 28), we again see different results using $\mu^{M}(t)$ for different exposure levels. There appears to be less of a need for a time dependent death rate as exposure levels increase. For the control and for 20ppm exposure, the time dependent $\mu^{M}$ appears to do a better job approximating the experimental data. However for an exposure level of 40ppm and especially $80 \mathrm{ppm}$, the time dependent $\mu^{M}$ does not appear to model the experimental data too differently from the optimal constant $\mu$. 

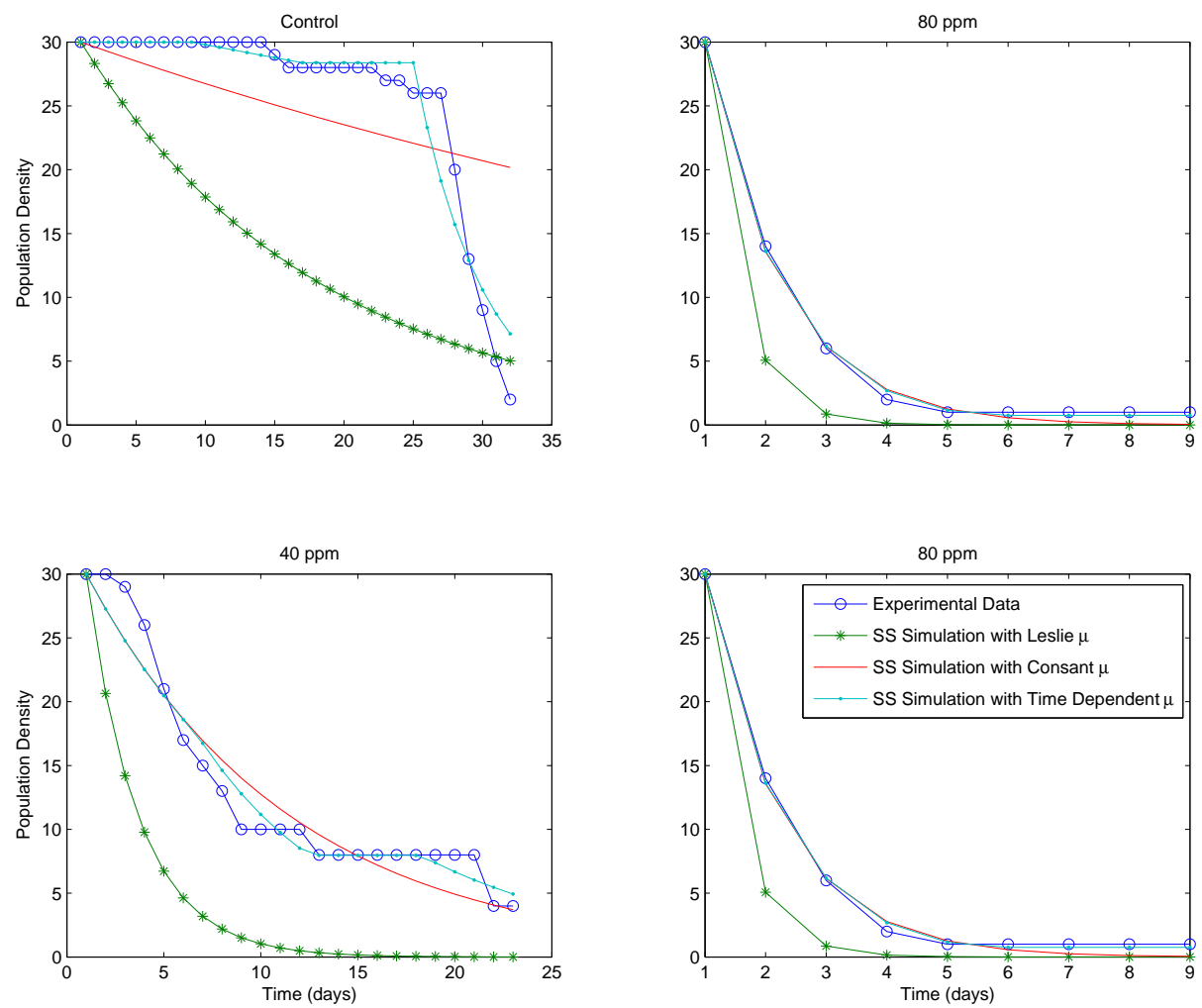

Figure 28: Comparisons of experimental data to SS simulations using Leslie $\mu$, the optimal constant $\mu$ and the optimal $\mu^{M}(t)$ with 4 nodes obtained for neonate exposure 

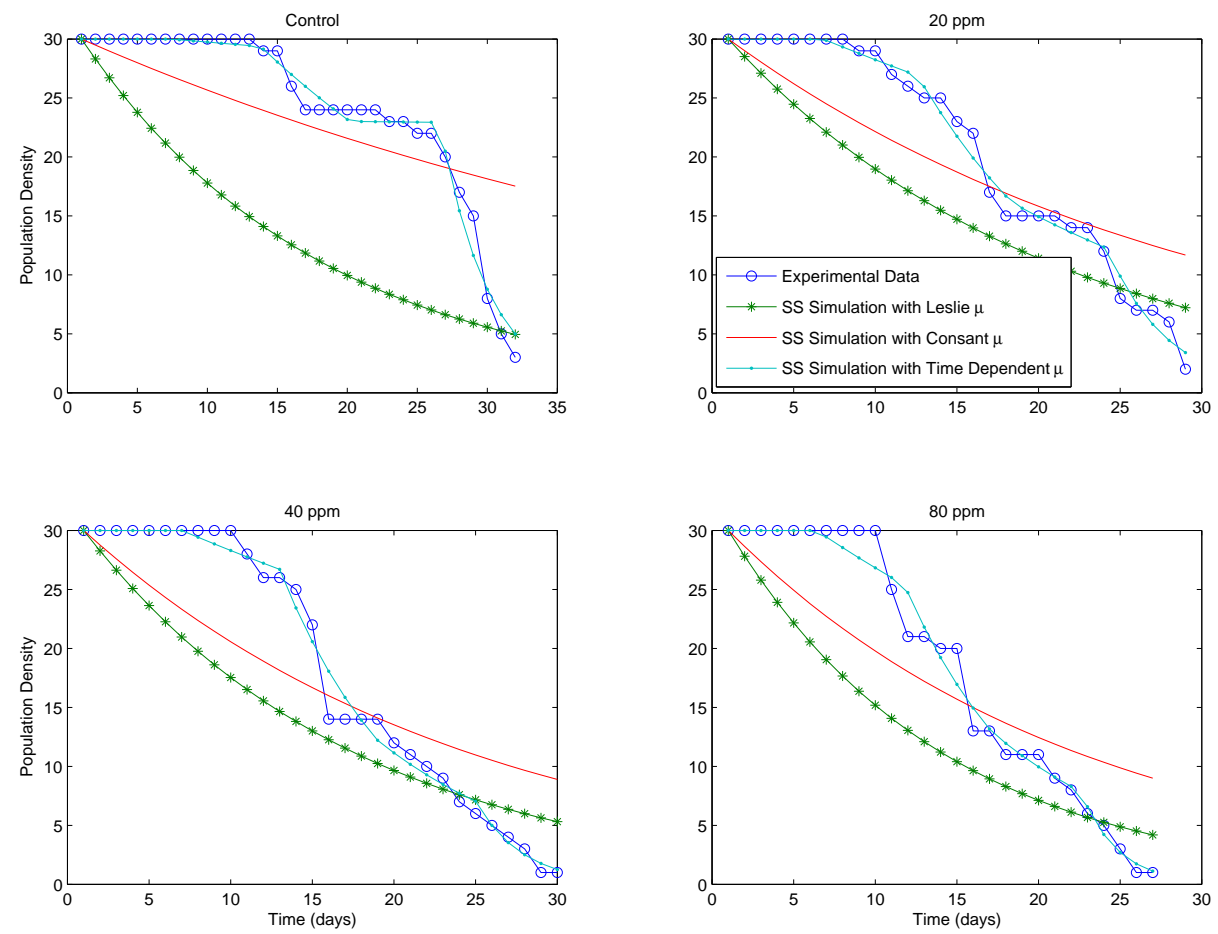

Figure 29: Comparisons of experimental data to SS simulations using Leslie $\mu$, the optimal constant $\mu$ and the optimal $\mu^{M}(t)$ with 5 nodes obtained for adult exposure 

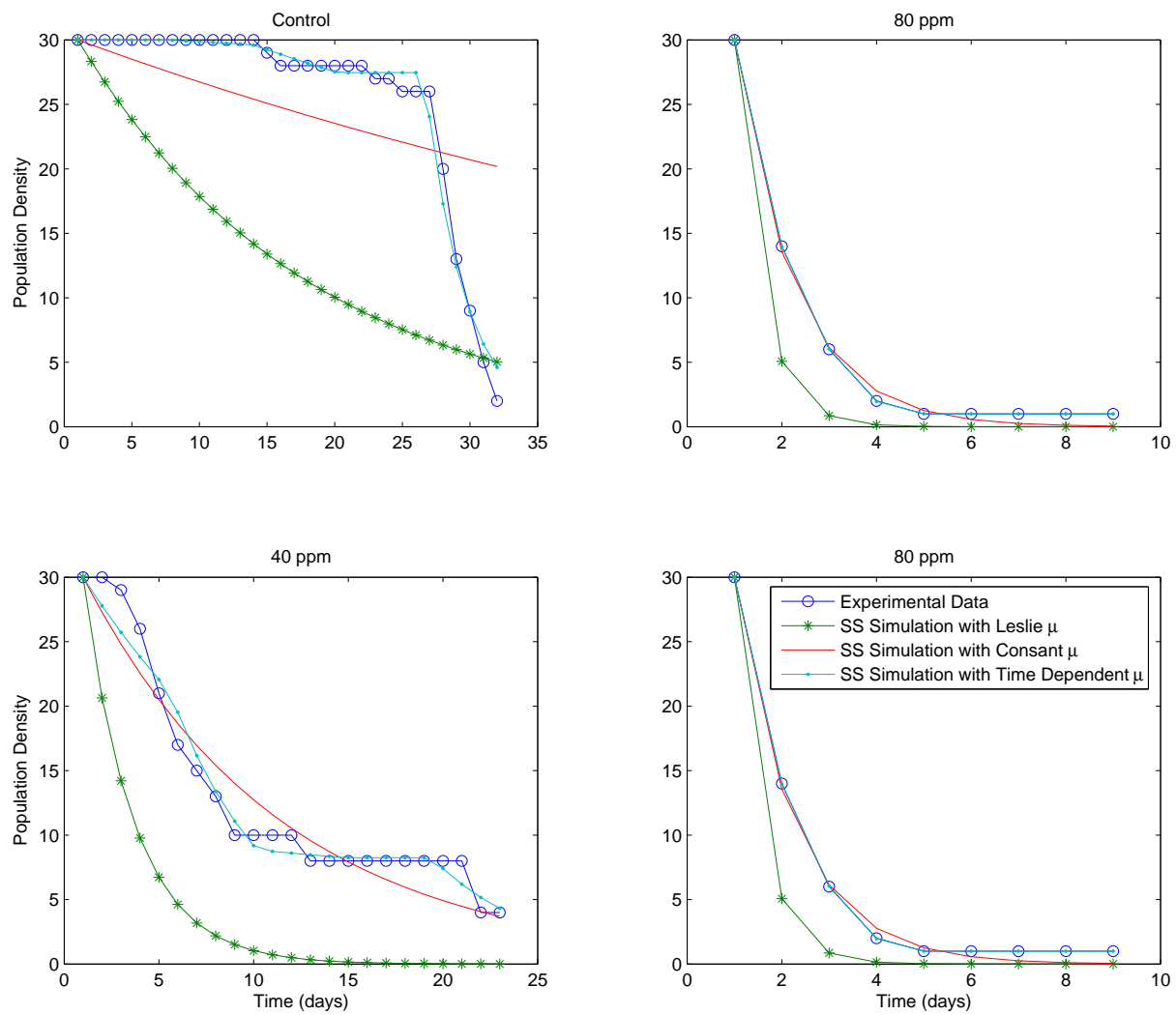

Figure 30: Comparisons of experimental data to SS simulations using Leslie $\mu$, the optimal constant $\mu$ and the optimal $\mu^{M}(t)$ with 5 nodes obtained for neonate exposure 

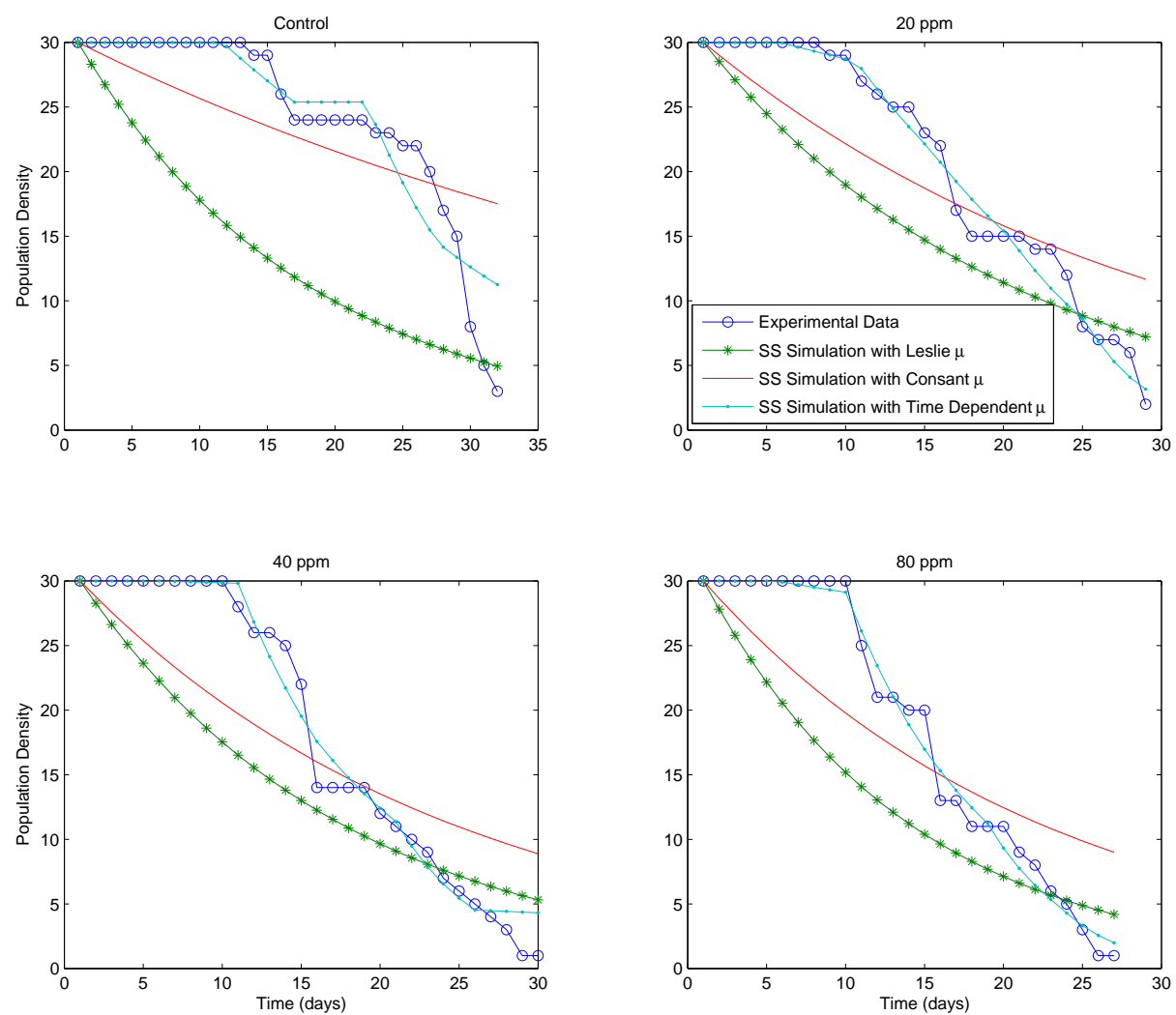

Figure 31: Comparisons of experimental data to SS simulations using Leslie $\mu$, the optimal constant $\mu$ and the optimal $\mu^{M}(t)$ with 6 nodes obtained for adult exposure 

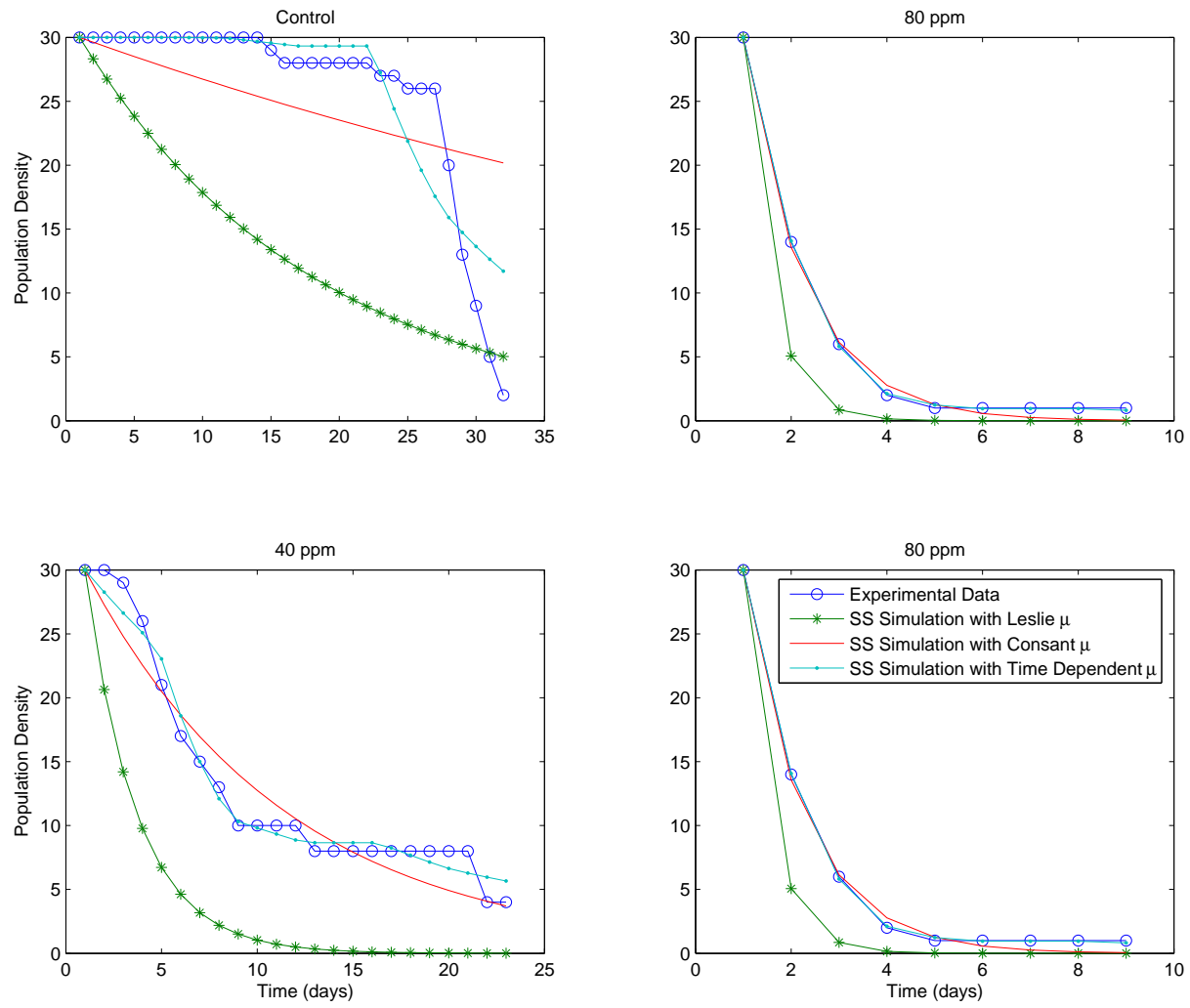

Figure 32: Comparisons of experimental data to SS simulations using Leslie $\mu$, the optimal constant $\mu$ and the optimal $\mu^{M}(t)$ with 6 nodes obtained for neonate exposure 
The results for adult and neonate exposure with $M=5$ (Figures 29 and 30) and $M=6$ (Figures 31 and 32) nodal values for $\mu^{M}(t)$ are very similar to those with $M=4$ nodal values. For adult exposure, our results seem to indicate that even as the exposure level increases, $\mu^{M}(t)$ as a time dependent variable more accurately models real life situations. However for neonate exposure, we see that as the exposure level increases, the fit to the experimental data does not appear to get much better. This is probably due to the fact that with neonate exposure at higher concentration levels, we see death in the population early in the experiment; the overall data resembles an exponential

decay. For 80ppm exposure, day 2 experiences drastic deaths that appear to continue as the population declines. Therefore it may be reasonable to consider death rate as a constant value for those exposure levels that exhibit substantial immediate deaths. In these cases sublethal damage is not an important factor in the population dynamics.

\subsubsection{Leslie Considerations}

One may ask how the SS Leslie trajectory compares with the actual Leslie matrix model output. The Leslie model output involves only the percent mortality (mort) value, which is not the death rate. The Leslie simulation is run, output received and finally used to compute the death rate. Therefore we do not expect that the output of SS using the Leslie mortality to be similar to that of the actual Leslie model output. For sake of comparison, graphs of these simulations are located in Figures 33 and 34. Henson $[\mathrm{H}]$ discusses the relationship between Leslie and the SS PDE models. She points out that "the Leslie model of choice in a given approximation is often low dimensional, and is hence too coarse a discretization to bear much realization to the associated PDE."

The figures manifest this hypothesis. The Leslie model does not do a very good job of estimating the experimental data. One may say that for the control, the Leslie model is exactly the data. This is true because mort $=0$ for both neonate and adult control. The Leslie model uses the experimental data in its calculations; therefore, for the lower pesticide concentrations it appears to approximate the data. However for all higher concentration levels, the Leslie model does not even come close to estimating the true values. The Leslie model performs even worse than the SS model with the Leslie computed death rate. This analysis leads us to again conclude that the discrete Leslie model is not an adequate population model for these sublethal dynamics. The SS PDE model does a much better job approximating the data. 

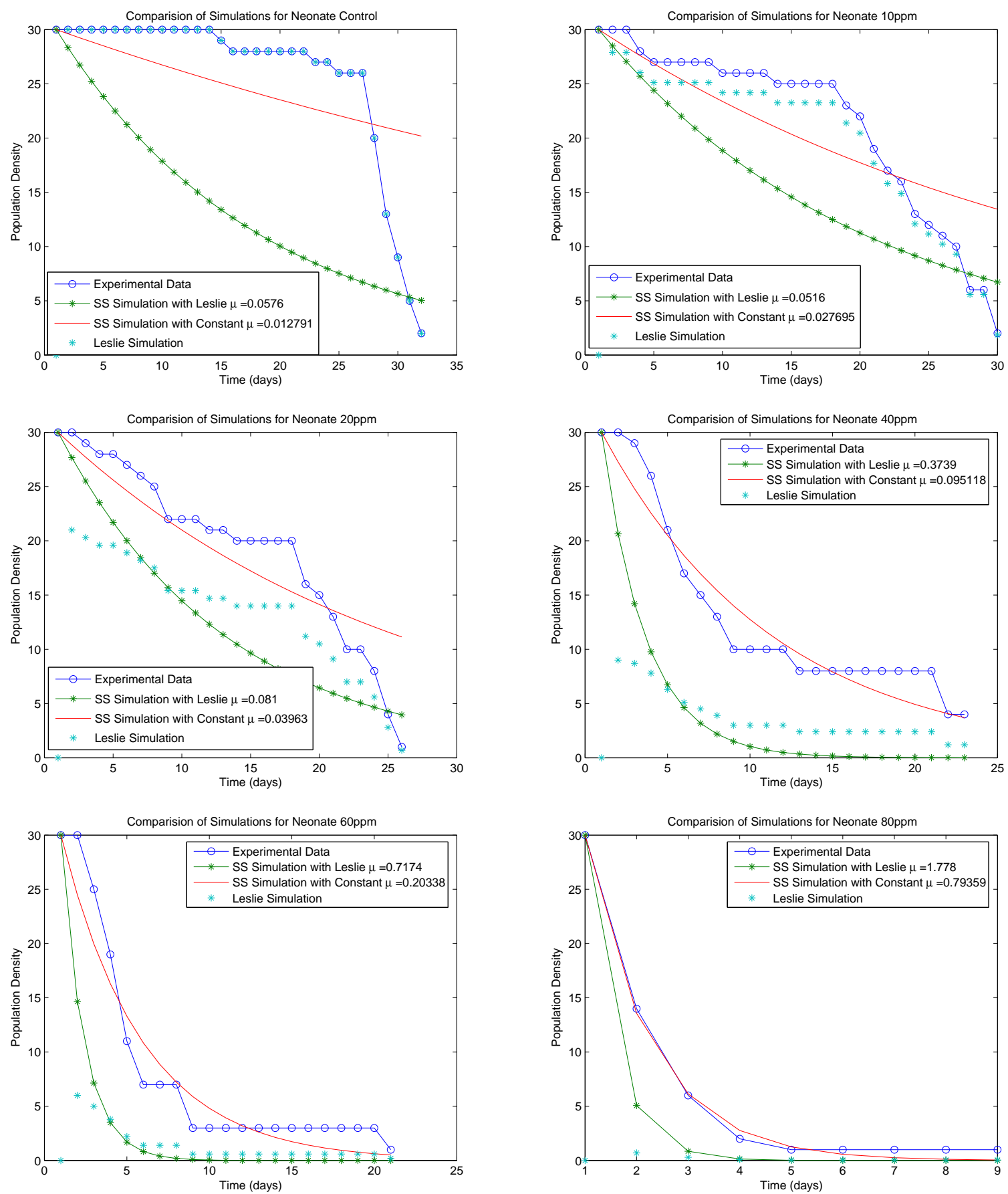

Figure 33: Comparison of Leslie and SS simulation for neonate exposure 

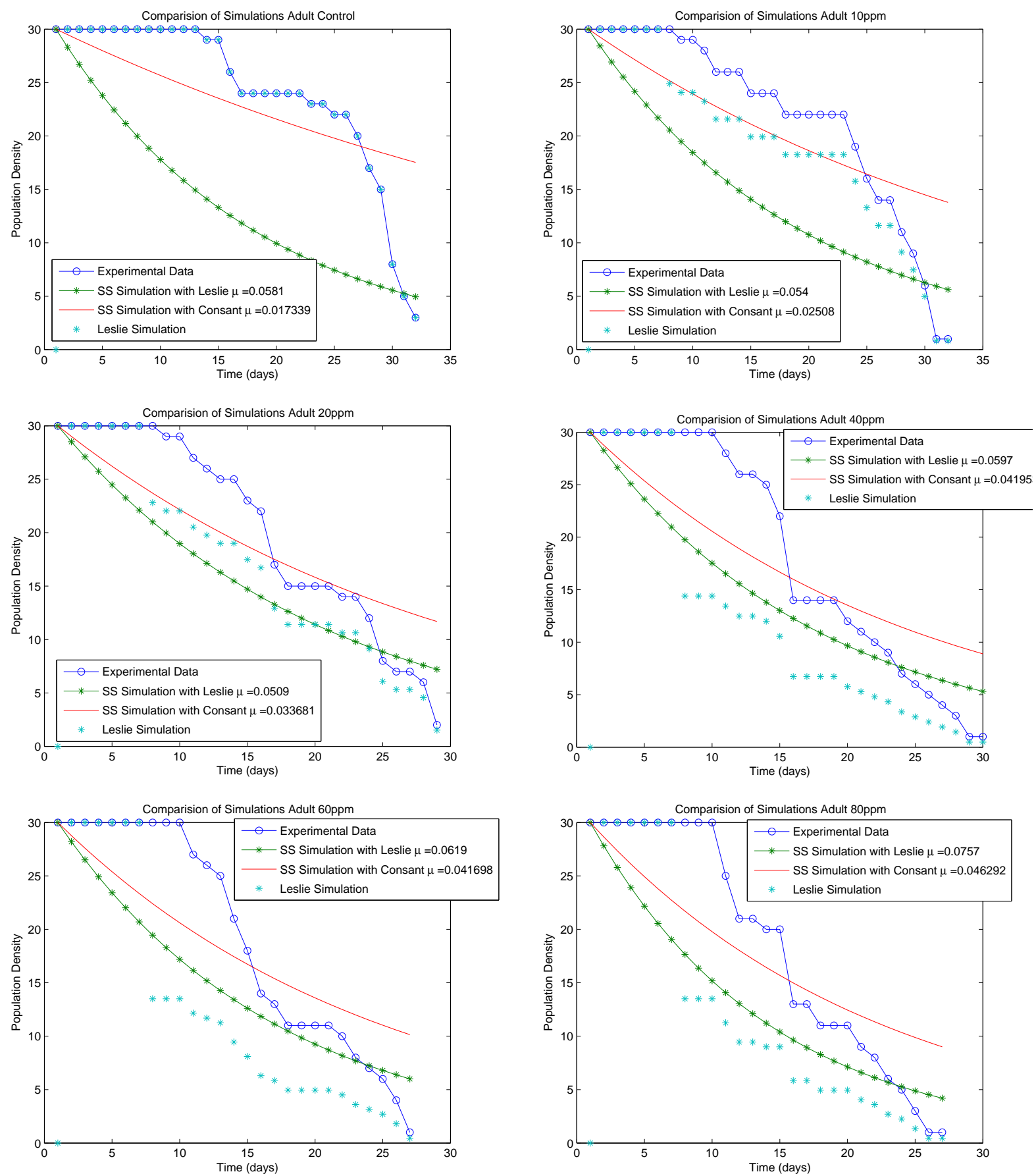

Figure 34: Comparisons of Leslie and SS simulation for adult exposure 


\subsubsection{Different Death Rates}

It is also of interest to consider the different death rates for adult and neonate exposure. Typical graphs of these different death rates with three nodal values for $\mu^{M}(t)$ can be found in Figures 35 $(20 \mathrm{ppm})$ and $36(60 \mathrm{ppm})$. We are able to see a large difference between the Leslie death rate, our
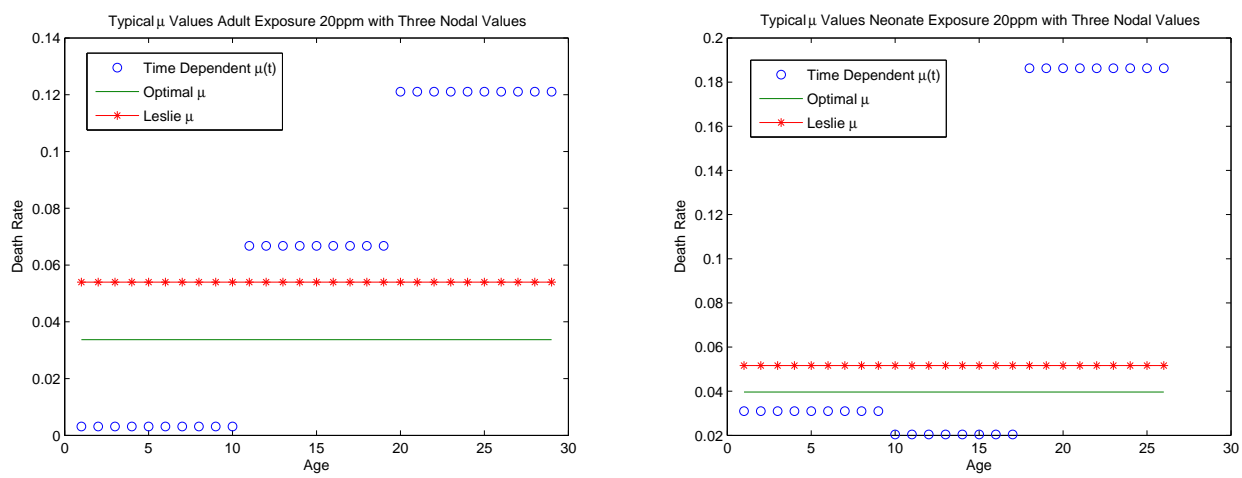

Figure 35: Comparisons of death rates - Leslie $\mu$, the optimal constant $\mu$ and the optimal $\mu^{M}(t)$ with $M=3$ for 20ppm adult exposure (right) and neonate exposure (left)
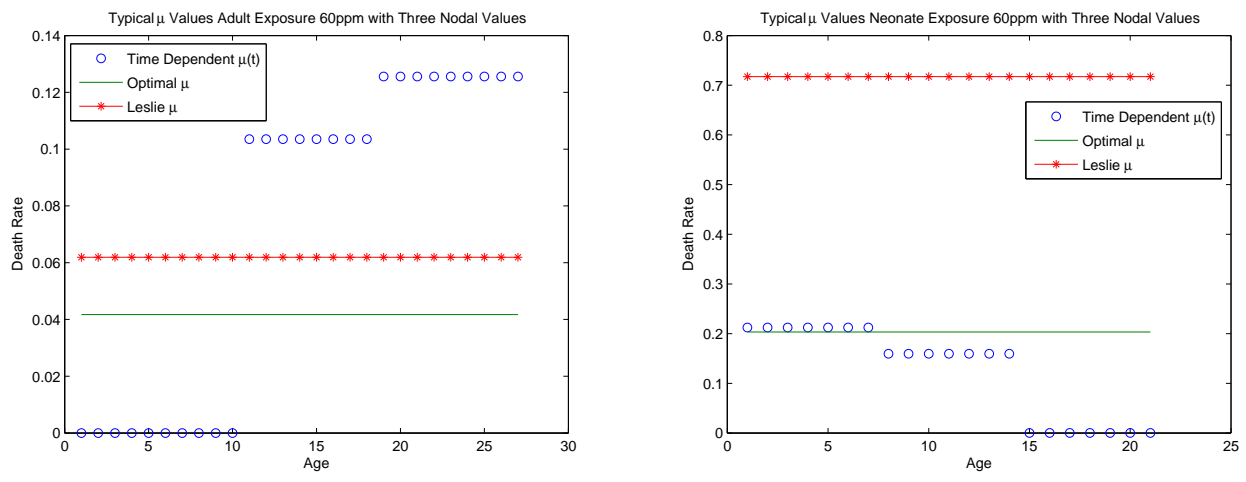

Figure 36: Comparisons of death rates - Leslie $\mu$, the optimal constant $\mu$ and the optimal $\mu^{M}(t)$ for 60ppm with $M=3$ adult exposure (right) and neonate exposure (left)

optimal constant death rate and our time dependent death rate.

Overall, our results strongly suggest that death rate should not be thought of as a constant value unless the concentration levels are so high that immediate death is certain. This only occurs for neonate exposure with high concentration levels, thus making a decision as to how to consider death rate (constant vs. non-constant) relatively straight forward. We should use death rate as a time dependent parameter when we wish to characterize the effects of sublethal damage on the aphids. 


\subsection{Determination of Confidence Intervals}

We would like to determine confidence intervals for each of our estimated parameters that would cover the true value. The parameter of interest is the death rate, $\mu^{M}(t)$, which we write as

$$
\mu^{M}(t) \approx\left\{a_{k}\right\}_{k=1}^{M}=\bar{a}
$$

where $M$ is the number of nodal values of $\mu^{M}(t)$.

Before we can compute confidence intervals, we will need to compute the standard deviation, $\hat{\sigma}^{2}$, and the sensitivity matrix, $F_{\bar{a}}$. Both of these values are computed using the experimental data and the model solution. Recall that we have experimental data along $(x, t)$, where $t=x-x_{0}$, thus we will consider $x=t+x_{0}$. The model solution we are concerned with is $U(t ; \bar{a})=U_{S S}\left(t+x_{0}, t ; \bar{a}\right)$, where $U_{S S}(x, t ; \bar{a})$ is the solution of the Sinko-Streifer equation with $\mu(t)$ approximated by

$$
\mu(t) \approx \mu^{M}(t)=\sum_{k=1}^{M} a_{k} \chi_{k}^{M}(t)
$$

where

$$
\chi_{k}^{M}= \begin{cases}1 & {\left[t_{k-1}^{M}, t_{k}^{M}\right)} \\ 0 & \text { elsewhere }\end{cases}
$$

Even though we begin simulations at $t_{0}=1$, we consider parameter approximations on $\left[0, t_{\text {max }}\right]$ and use partitions $t_{k}^{M}=\frac{t_{\max } k}{M}$. Here and below we suppress the notation indicating that $U_{S S}$ depends on $M$. The standard deviation is approximated as

$$
\sigma^{2} \approx \hat{\sigma}^{2}=\frac{1}{n-M} \sum_{j=1}^{n}\left(U\left(t_{j}, \bar{a}\right)-U_{j}^{d}\right)^{2},
$$

with $n$ representing the number of data points, $M$ representing the number of nodal values of $\bar{a}$, and $U_{j}^{d}$ representing the experimental data. The sensitivity matrix $F_{\bar{a}}(n \times M)$ is computed in the following manner:

$$
F_{\bar{a}}=\left(\begin{array}{c}
F_{\bar{a}}\left(t_{0}\right) \\
\vdots \\
F_{\bar{a}}\left(t_{n}\right)
\end{array}\right)=\left(\frac{\partial U\left(t_{j}, \bar{a}\right)}{\partial a_{k}}\right)=\left(\begin{array}{ccc}
\frac{\partial U\left(t_{1}\right)}{\partial a_{1}} & \ldots & \frac{\partial U\left(t_{1}\right)}{\partial a_{M}} \\
\vdots & \ddots & \vdots \\
\frac{\partial U\left(t_{n}\right)}{\partial a_{1}} & \ldots & \frac{\partial U\left(t_{n}\right)}{\partial a_{M}}
\end{array}\right)=\left(F_{\bar{a}}\right)_{j k}
$$

We assume a statistical model for the observation process given by: $Y_{j}=U\left(t_{j}, \bar{a}\right)+\epsilon_{j}$, with $\bar{a} \in$ $R^{M}$ and $\epsilon_{j}$ normally distributed $\mathcal{N}\left(0, \sigma^{2}\right)$. The formula for the confidence intervals $(k=1, \ldots, M)$ is given by $[\mathrm{WMS}]$ as

$$
\left[a_{k}-t_{1-\frac{\alpha}{2}} S E\left(a_{k}\right), a_{k}+t_{1-\frac{\alpha}{2}} S E\left(a_{k}\right)\right]
$$


where $t_{1-\frac{\alpha}{2}}$ is a distribution value obtained from a statistical chart and the standard errors, $S E\left(a_{k}\right)$, are determined by the formula

$$
S E\left(a_{k}\right)=\sqrt{\Sigma_{k k}},
$$

where $\Sigma_{k k}$ are the diagonal elements of the covariance matrix, $\Sigma$, approximated by

$$
\Sigma \approx \hat{\sigma}^{2}\left[F_{\bar{a}}^{T} F_{\bar{a}}\right]^{-1}
$$

We will now use the above derivations to compute confidence intervals for our problem. Recall, for example, the SS solution when $M=4$. We have

$$
U(t ; \bar{a})= \begin{cases}\Phi\left(x_{0}\right) e^{-a_{1} t} & {\left[0, t_{1}^{M}\right)} \\ \Phi\left(x_{0}\right) e^{\left(a_{2}-a_{1}\right) t_{1}^{M}} e^{-a_{2} t} & {\left[t_{1}^{M}, t_{2}^{M}\right)} \\ \Phi\left(x_{0}\right) e^{\left(a_{3}-a_{2}\right) t_{2}^{M}} e^{\left(a_{2}-a_{1}\right) t_{1}^{M}} e^{-a_{3} t} & {\left[t_{2}^{M}, t_{3}^{M}\right)} \\ \Phi\left(x_{0}\right) e^{\left(a_{4}-a_{3}\right) t_{3}^{M}} e^{\left(a_{3}-a_{2}\right) t_{2}^{M}} e^{\left(a_{2}-a_{1}\right) t_{1}^{M}} e^{-a_{4} t} & {\left[t_{3}^{M}, t\right) .}\end{cases}
$$

Thus, the sensitivity matrix, $F_{\bar{a}}$, is given by

$$
F_{\bar{a}}=\left(\frac{\partial U\left(t_{j}, \bar{a}\right)}{\partial a_{k}}\right)
$$

where

$$
\begin{aligned}
& \frac{\partial U}{\partial a_{1}}= \begin{cases}-t U(t: \bar{a}) & {\left[0, t_{1}^{M}\right)} \\
-t_{1}^{M} U(t: \bar{a}) & {\left[t_{1}^{M}, t\right)}\end{cases} \\
& \frac{\partial U}{\partial a_{2}}= \begin{cases}0 & {\left[0, t_{1}^{M}\right)} \\
\left(t_{1}^{M}-t\right) U(t: \bar{a}) & {\left[t_{1}^{M}, t_{2}^{M}\right)} \\
\left(t_{1}^{M}-t_{2}^{M}\right) U(t: \bar{a}) & {\left[t_{2}^{M}, t\right)}\end{cases} \\
& \frac{\partial U}{\partial a_{3}}= \begin{cases}0 & {\left[0, t_{2}^{M}\right)} \\
\left(t_{2}^{M}-t\right) U(t: \bar{a}) & {\left[t_{2}^{M}, t_{3}^{M}\right)} \\
\left(t_{2}^{M}-t_{3}^{M}\right) U(t: \bar{a}) & {\left[t_{3}^{M}, t\right)}\end{cases} \\
& \frac{\partial U}{\partial a_{4}}= \begin{cases}0 & {\left[0, t_{3}^{M}\right)} \\
\left(t_{3}^{M}-t\right) U(t: \bar{a}) & {\left[t_{3}^{M}, t\right)}\end{cases}
\end{aligned}
$$

Once we obtain $F_{\bar{a}}$, we can compute the standard errors $S E$. To complete the confidence interval calculations, a level of significance must be chosen. We choose $\alpha=.025$, which due to the symmetry of the t-distribution, corresponds to a significance level of $95 \%$. Thus we are confident that $95 \%$ of the time our estimation procedure will yield confidence intervals that cover the nodal values of the "true" values of $\mu^{M}(t)$. As usual in statistics, this tacitly assumes the existence of a "true" $\mu^{M}(t)$. (Here, as in most applications of this theory, this assumption is almost certainly not true!) When the number of data points is large, $n \geq 30$ the t-value is 1.96 . However we have 
Table 12: t-values for $M=4$

\begin{tabular}{|l|cc|cc|}
\hline & \multicolumn{2}{|c|}{$\begin{array}{c}\text { Neonate } \\
\mathbf{n}\end{array}$} & \multicolumn{2}{c|}{ Adult } \\
t-value & $\mathbf{n}$ & t-value \\
\hline \hline Control & 32 & 1.96 & 32 & 1.96 \\
\hline 10 ppm & 30 & 1.96 & 32 & 1.96 \\
\hline 20 ppm & 26 & 2.074 & 29 & 2.060 \\
\hline 40 ppm & 23 & 2.093 & 30 & 1.96 \\
\hline 60 ppm & 21 & 2.110 & 27 & 2.069 \\
\hline 80 ppm & 9 & 2.571 & 27 & 2.069 \\
\hline
\end{tabular}

different $n$ values for our different exposure levels and methods. A chart of the t-values used for $M=4$ is given in Table 12. Similar charts were constructed for other values of $M$ but will not be given here.

The above method was utilized to determine confidence intervals for all exposure levels and methods with $\bar{a}^{M}$ where $M=1,2, \ldots, 6$. The results for neonate exposure can be found in Table 13 and in Figures (37-48). The results for adult exposure can be found in Table 14 and Figures (49-60). The tables contain the actual confidence intervals, while the figures contain the population densities computed using the optimal $\mu^{M}(t)$ and the upper and lower bounds on $\mu^{M}(t)$, and the error bars on $\mu^{M}(t)$. The upper bounds on $\mu^{M}(t)$ are the SS simulations computed using the highest death rate resulting from the confidence interval calculations (highest value of error bar) while the lower bounds on $\mu^{M}(t)$ are the SS simulations computed using the lowest death rate (lowest value of error bar) resulting from the confidence interval calculations. High death rates produce the "lower band" of simulations; low death rates produce the "high band" of simulations. 
Table 13: Confidence intervals for neonate exposure: $\overline{a_{k}} \pm t_{1-\frac{\alpha}{2}} S E\left(a_{k}\right)$

\begin{tabular}{|c|c|c|c|}
\hline & Control & 10ppm & 20ppm \\
\hline \hline $\mathbf{M = 1}$ & $.0128 \pm .0188$ & $.0277 \pm .0223$ & $.0296 \pm .0255$ \\
\hline $\mathbf{M = 2}$ & $0 \pm .0080$ & $0 \pm .0046$ & $.0253 \pm .0080$ \\
& $.0501 \pm .0044$ & $.0870 \pm .0029$ & $.0897 \pm .0041$ \\
\hline $\mathbf{M = 3}$ & $.0013 \pm .0160$ & $.0134 \pm .0052$ & $.0310 \pm .0075$ \\
& $0 \pm .0039$ & $.0181 \pm .0021$ & $.0240 \pm .0032$ \\
& $.1112 \pm .0079$ & $.1658 \pm .0044$ & $.1863 \pm .0057$ \\
\hline $\mathbf{M = 4}$ & $0 \pm .0188$ & $.0180 \pm .0068$ & $.0270 \pm .0108$ \\
& $.0070 \pm .0043$ & $.0010 \pm .0028$ & $.0328 \pm .0051$ \\
& $0 \pm .0053$ & $.0613 \pm .0039$ & $.0345 \pm .0068$ \\
& $.1972 \pm .0095$ & $.2031 \pm .0079$ & $.2799 \pm .0104$ \\
\hline $\mathbf{M = 5}$ & $0 \pm .0069$ & $.0186 \pm .0078$ & $.0203 \pm .0106$ \\
& $.0018 \pm .0028$ & $.0065 \pm .0029$ & $.0515 \pm .0052$ \\
& $.0120 \pm .0032$ & $.0124 \pm .0036$ & $0 \pm .0068$ \\
& $0 \pm .0035$ & $.1080 \pm .0049$ & $.0978 \pm .0090$ \\
& $.3307 \pm .0078$ & $.2184 \pm .0098$ & $.3204 \pm .0135$ \\
\hline $\mathbf{M = 6}$ & $0 \pm .0320$ & $.0196 \pm .0177$ & $.0165 \pm .0266$ \\
& $.0003 \pm .0130$ & $.0089 \pm .0064$ & $.0526 \pm .0127$ \\
& $.0040 \pm .0162$ & $.0037 \pm .0079$ & $.0223 \pm .0177$ \\
& $0 \pm .0154$ & $.0411 \pm .0088$ & $.0030 \pm .0176$ \\
& $.1098 \pm .0205$ & $.1550 \pm .0130$ & $.1953 \pm .0267$ \\
& $.0768 \pm .0335$ & $.0805 \pm .0155$ & $.0138 \pm .0244$ \\
\hline
\end{tabular}

\begin{tabular}{|l|c|c|c|}
\hline & 40ppm & 60ppm & 80ppm \\
\hline \hline $\mathbf{M = 1}$ & $.0951 \pm .0582$ & $.2034 \pm .5027$ & $.7174 \pm 3.5263$ \\
\hline $\mathbf{M = 2}$ & $.1057 \pm .0141$ & $.2127 \pm .0346$ & $.7959 \pm .0153$ \\
& $.0385 \pm .0043$ & $.0300 \pm .0054$ & $0 \pm .0010$ \\
\hline $\mathbf{M = 3}$ & $.1087 \pm .0219$ & $.2133 \pm .0463$ & $.8059 \pm .0700$ \\
& $.0729 \pm .0137$ & $.1595 \pm .0459$ & $.4217 \pm .0139$ \\
& $.0406 \pm .0126$ & $0 \pm .0284$ & $0 \pm .0083$ \\
\hline $\mathbf{M = 4}$ & $.0956 \pm .0257$ & $.1987 \pm .0479$ & $.7895 \pm .0598$ \\
& $.1350 \pm .0160$ & $.2693 \pm .0535$ & $.8492 \pm .0235$ \\
& $0 \pm .0245$ & $0 \pm .1108$ & $0 \pm .0282$ \\
& $.1010 \pm .0203$ & $.0394 \pm .0654$ & $0 \pm .0150$ \\
\hline $\mathbf{M = 5}$ & $.0769 \pm .0247$ & $.1754 \pm .0487$ & $.7646 \pm .0069$ \\
& $.1888 \pm .0153$ & $.3769 \pm .0556$ & $1.1222 \pm .0067$ \\
& $.0151 \pm .0282$ & $0 \pm .1249$ & $.0031 \pm .0051$ \\
& $0 \pm .0301$ & $0 \pm .1453$ & $0 \pm .0034$ \\
& $.1797 \pm .0248$ & $.1369 \pm .0824$ & $0 \pm .0018$ \\
\hline $\mathbf{M = 6}$ & $.0596 \pm .0304$ & $.1429 \pm .0518$ & $.7568 \pm .0505$ \\
& $.2146 \pm .0162$ & $.4582 \pm .0497$ & $1.0099 \pm .0599$ \\
& $.0502 \pm .0289$ & $.0528 \pm .1239$ & $.5365 \pm .0438$ \\
& $0 \pm .0333$ & $0 \pm .1700$ & $0 \pm .0357$ \\
& $.0722 \pm .0379$ & $.0495 \pm .1690$ & $0 \pm .0302$ \\
& $.0523 \pm .0249$ & $.0399 \pm .0805$ & $.2835 \pm .0141$ \\
\hline
\end{tabular}



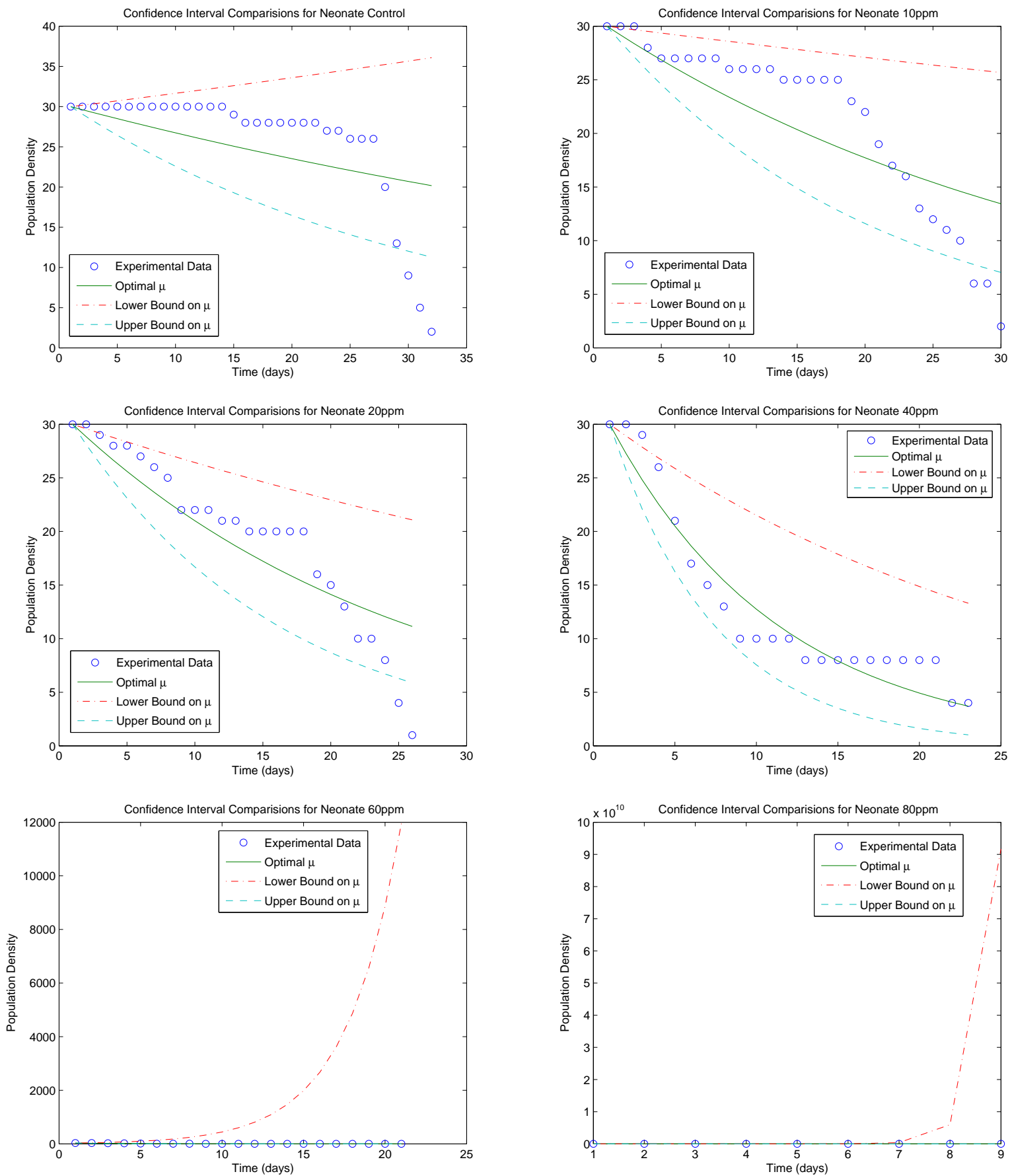

Figure 37: Population densities for neonate exposure run with upper and lower bounds of confidence intervals $(\mathrm{M}=1)$ 

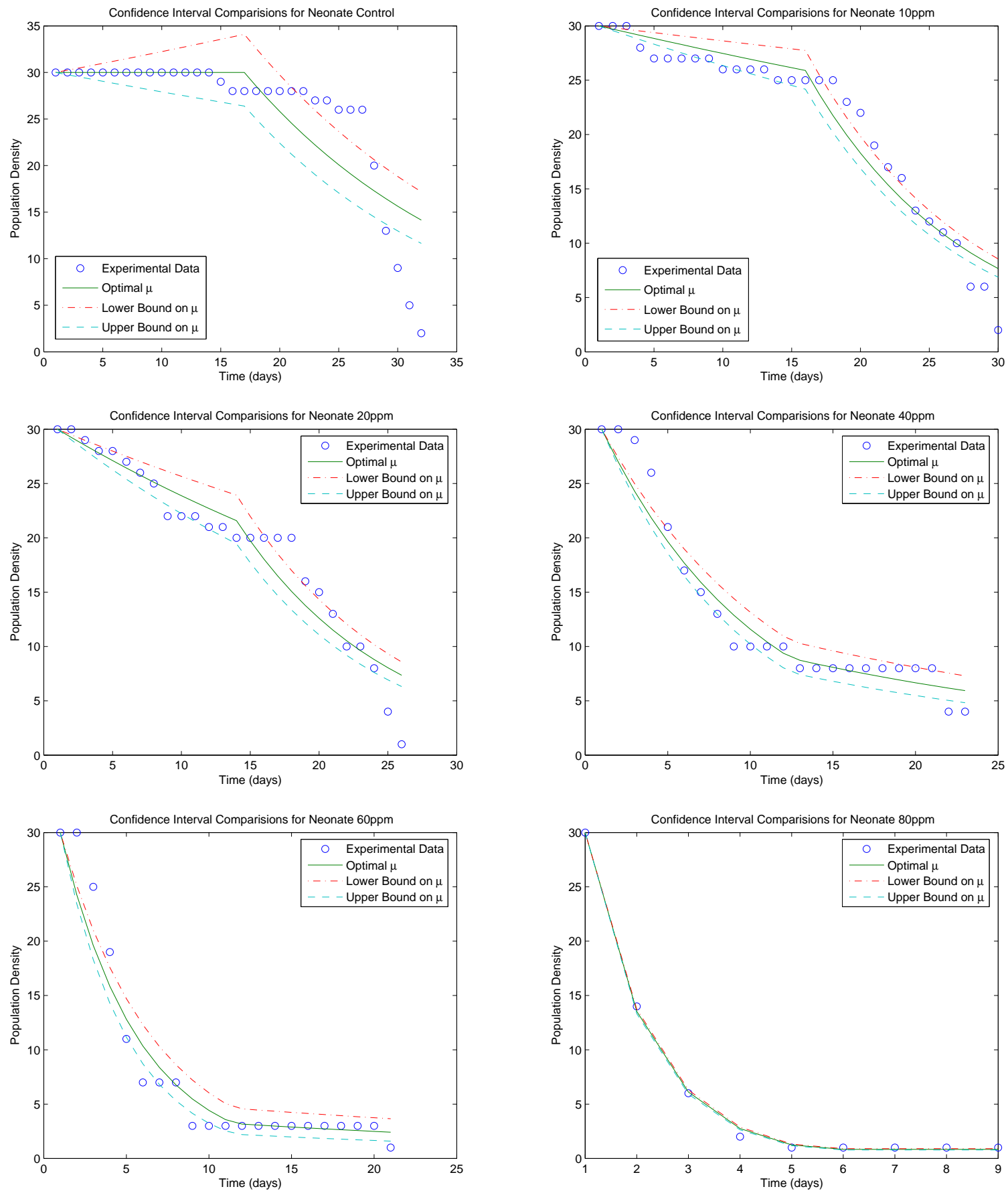

Figure 38: Population densities for neonate exposure run with upper and lower bounds of confidence intervals $(\mathrm{M}=2)$ 

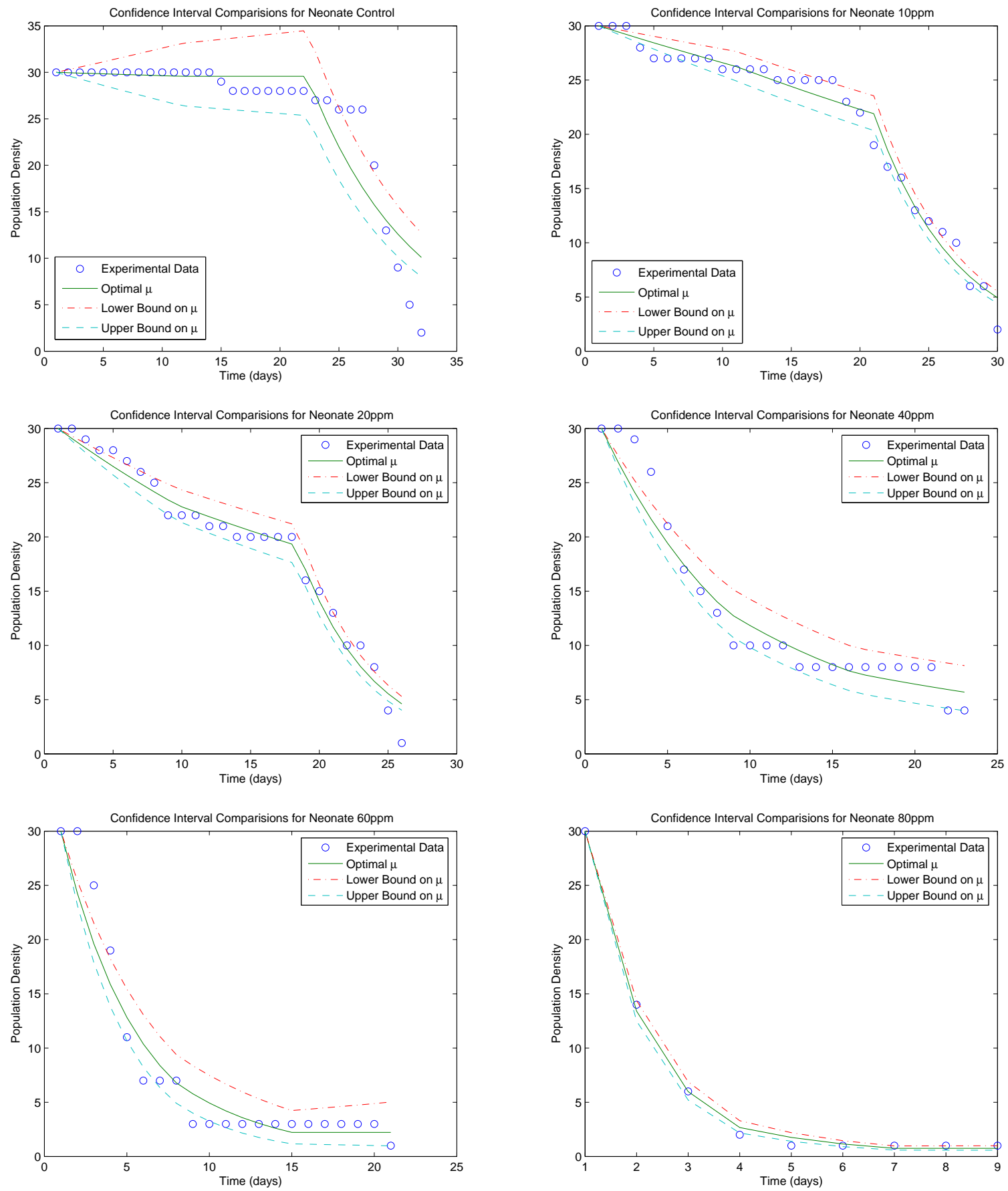

Figure 39: Population densities for neonate exposure run with upper and lower bounds of confidence intervals $(\mathrm{M}=3)$ 

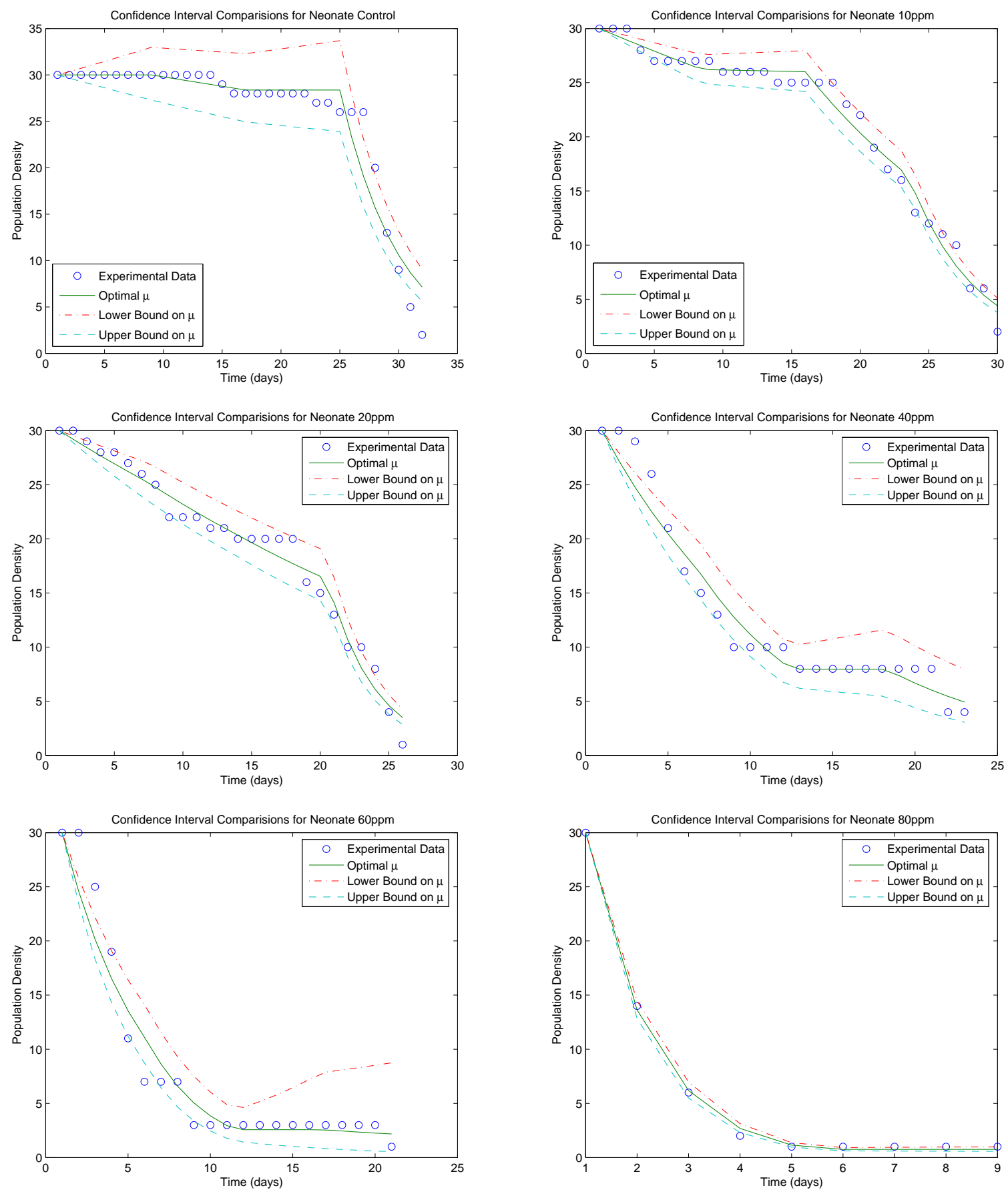

Figure 40: Population densities for neonate exposure run with upper and lower bounds of confidence intervals $(\mathrm{M}=4)$ 

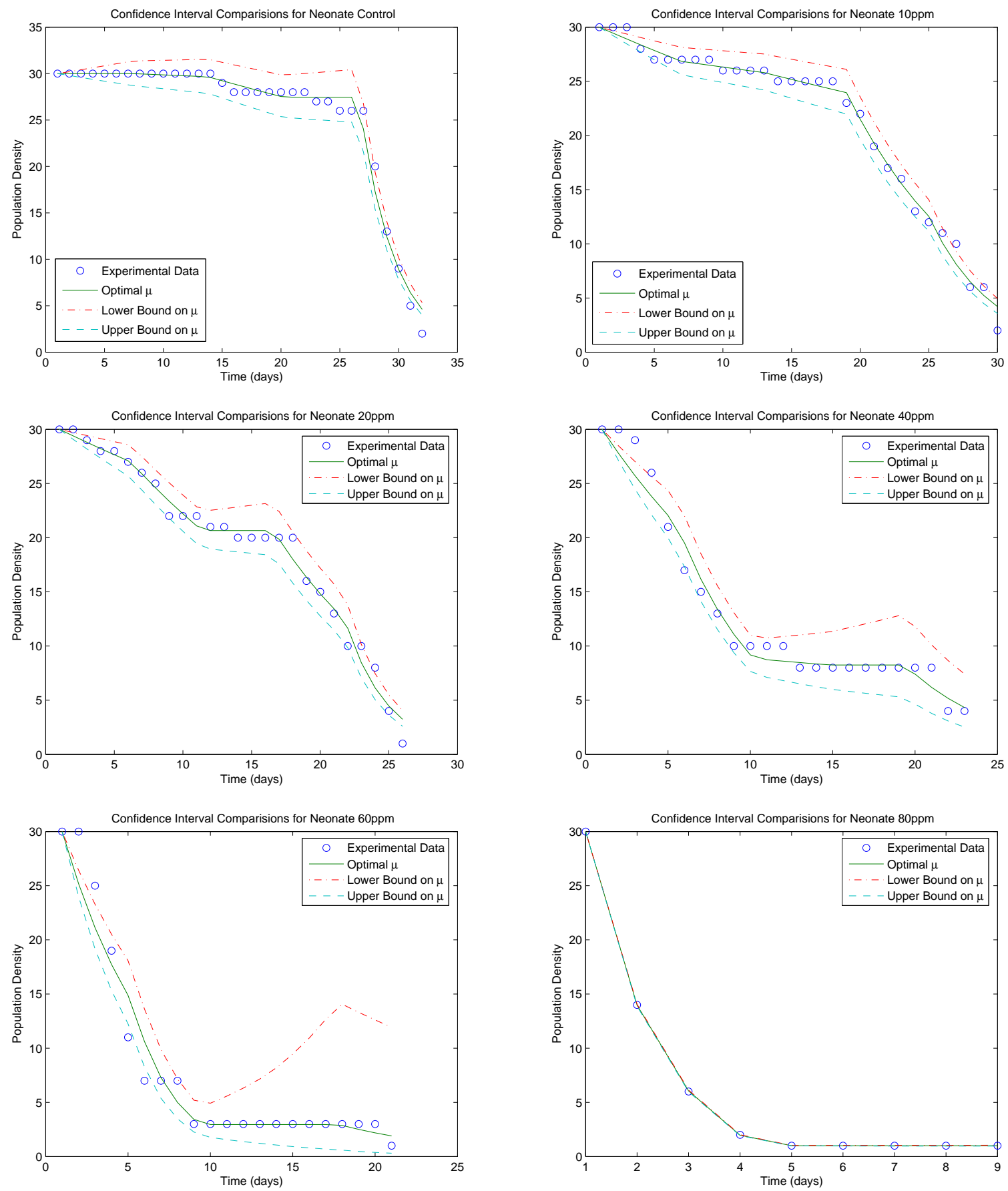

Figure 41: Population densities for neonate exposure run with upper and lower bounds of confidence intervals $(\mathrm{M}=5)$ 

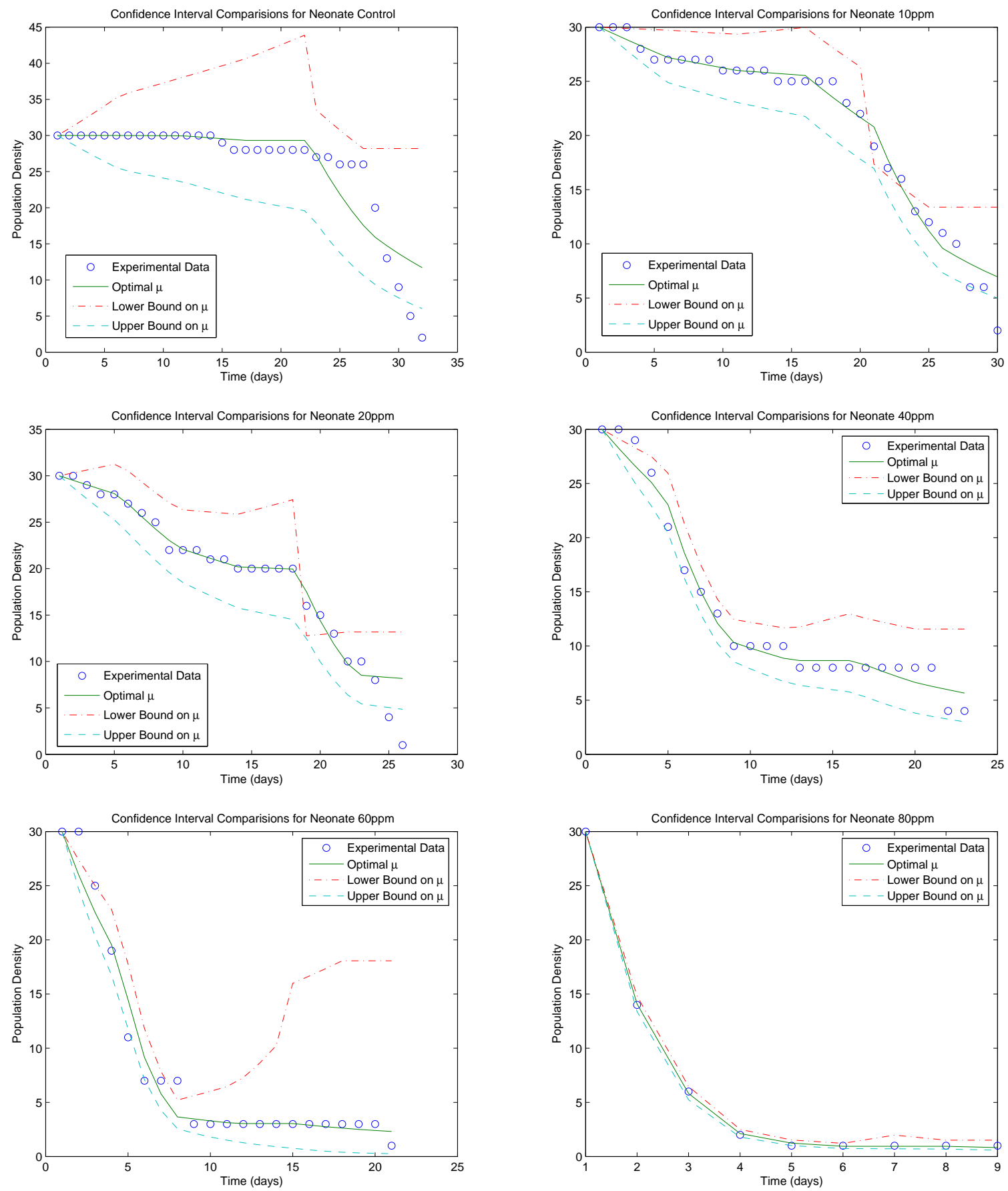

Figure 42: Population densities for neonate exposure run with upper and lower bounds of confidence intervals $(\mathrm{M}=6)$ 

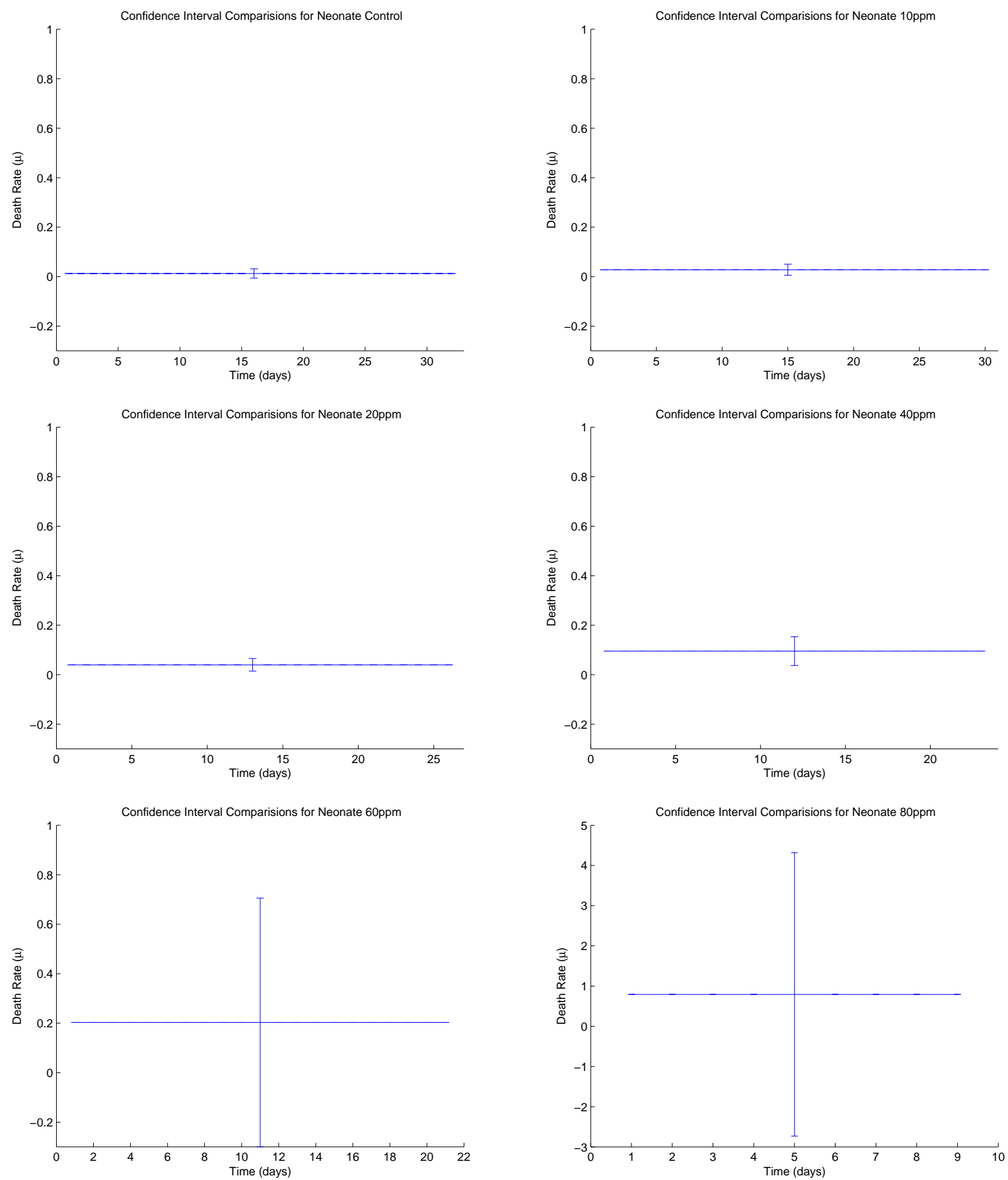

Figure 43: Confidence intervals on $\mu$ for $\mathrm{M}=1$ neonate exposure 

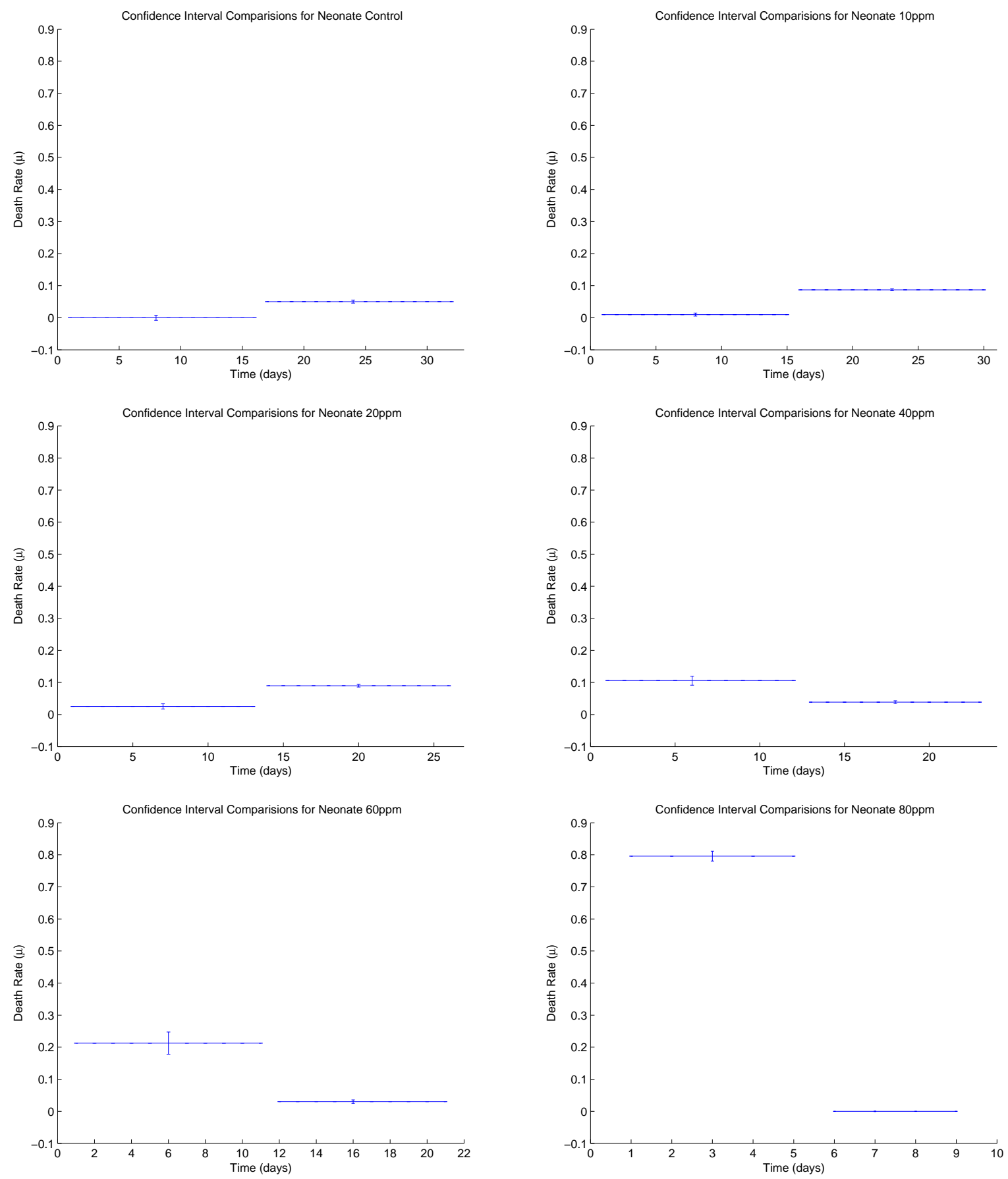

Figure 44: Confidence intervals on $\mu$ for $\mathrm{M}=2$ neonate exposure 

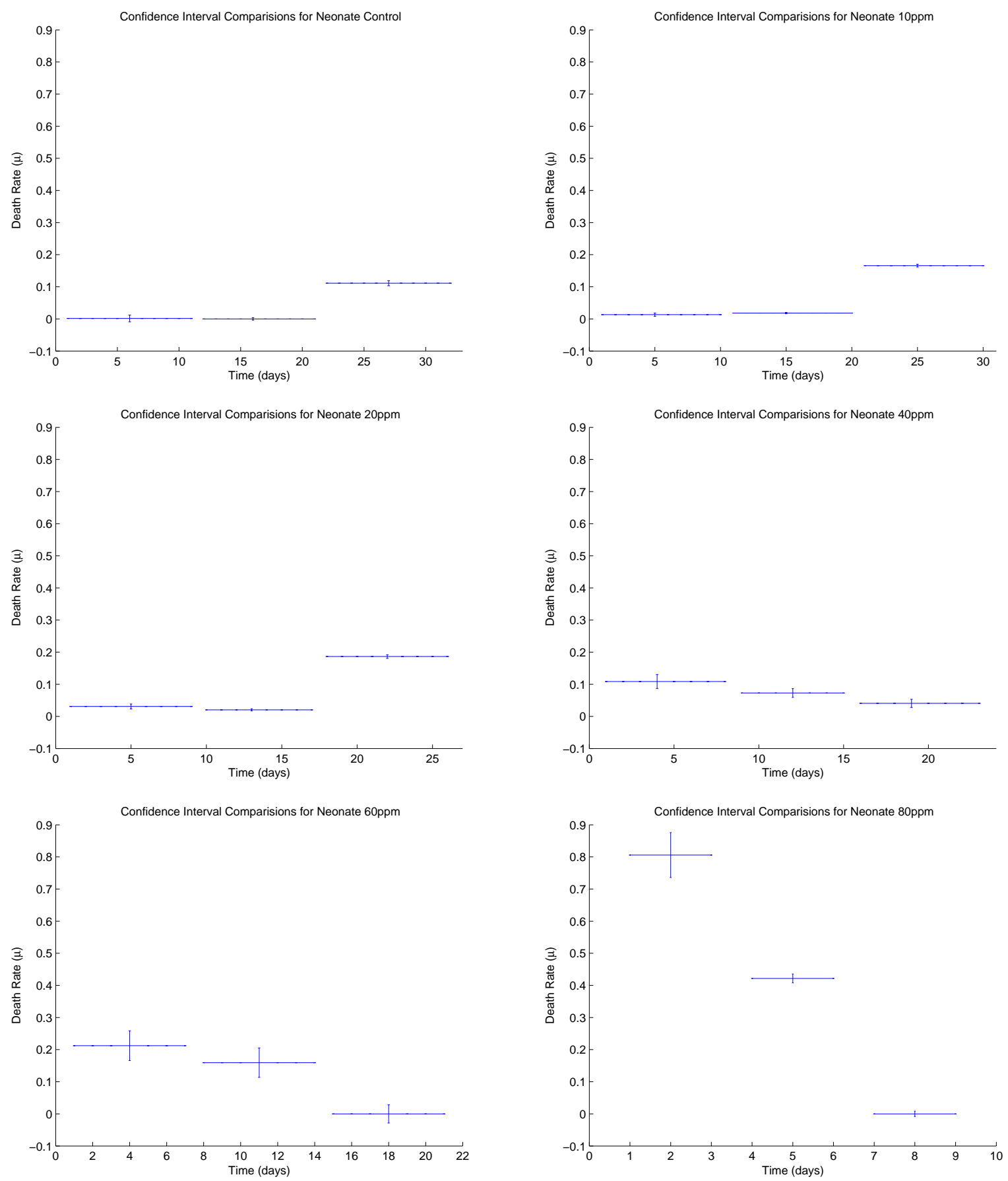

Figure 45: Confidence intervals on $\mu$ for $\mathrm{M}=3$ neonate exposure 

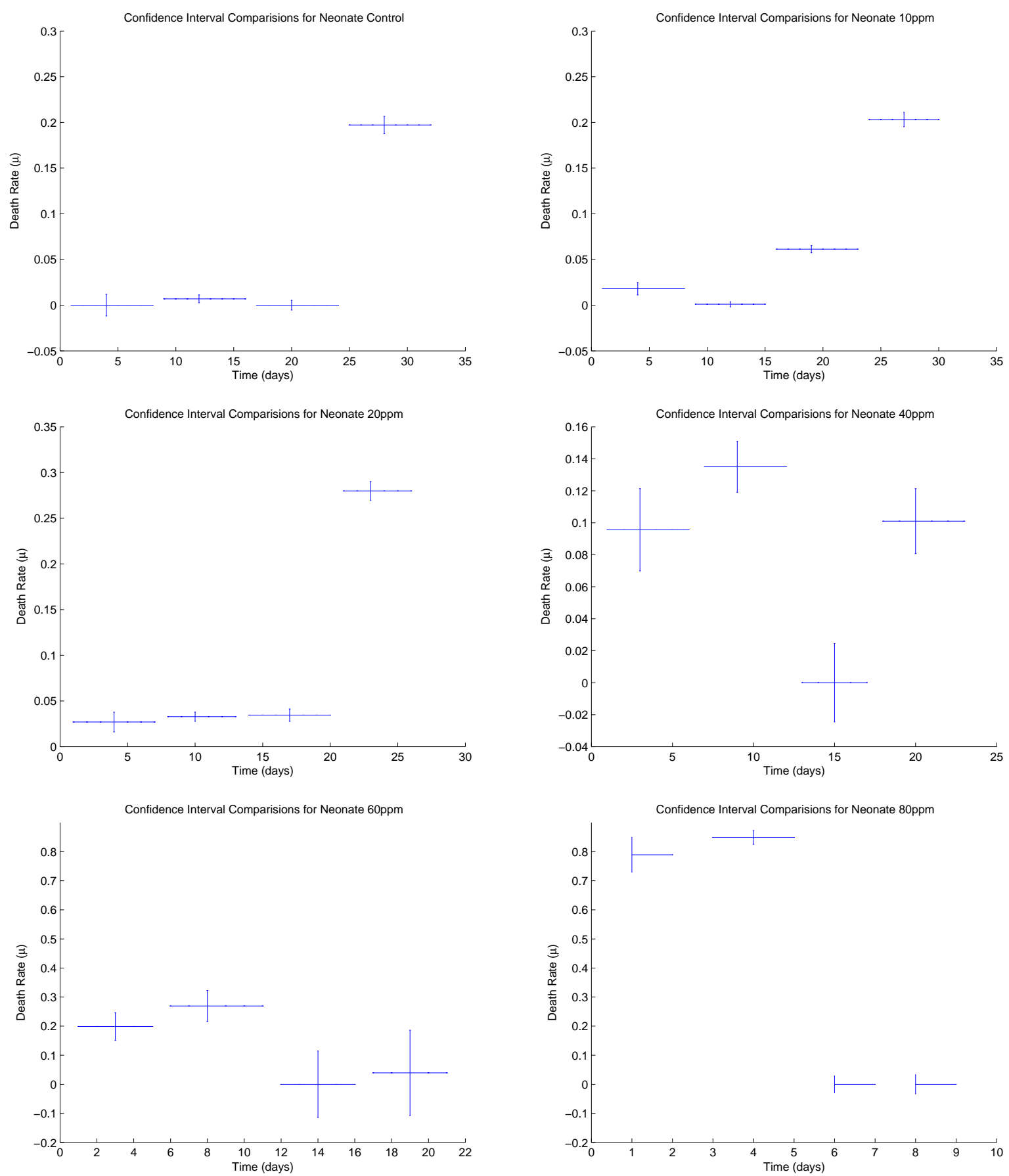

Figure 46: Confidence intervals on $\mu$ for $\mathrm{M}=4$ neonate exposure 

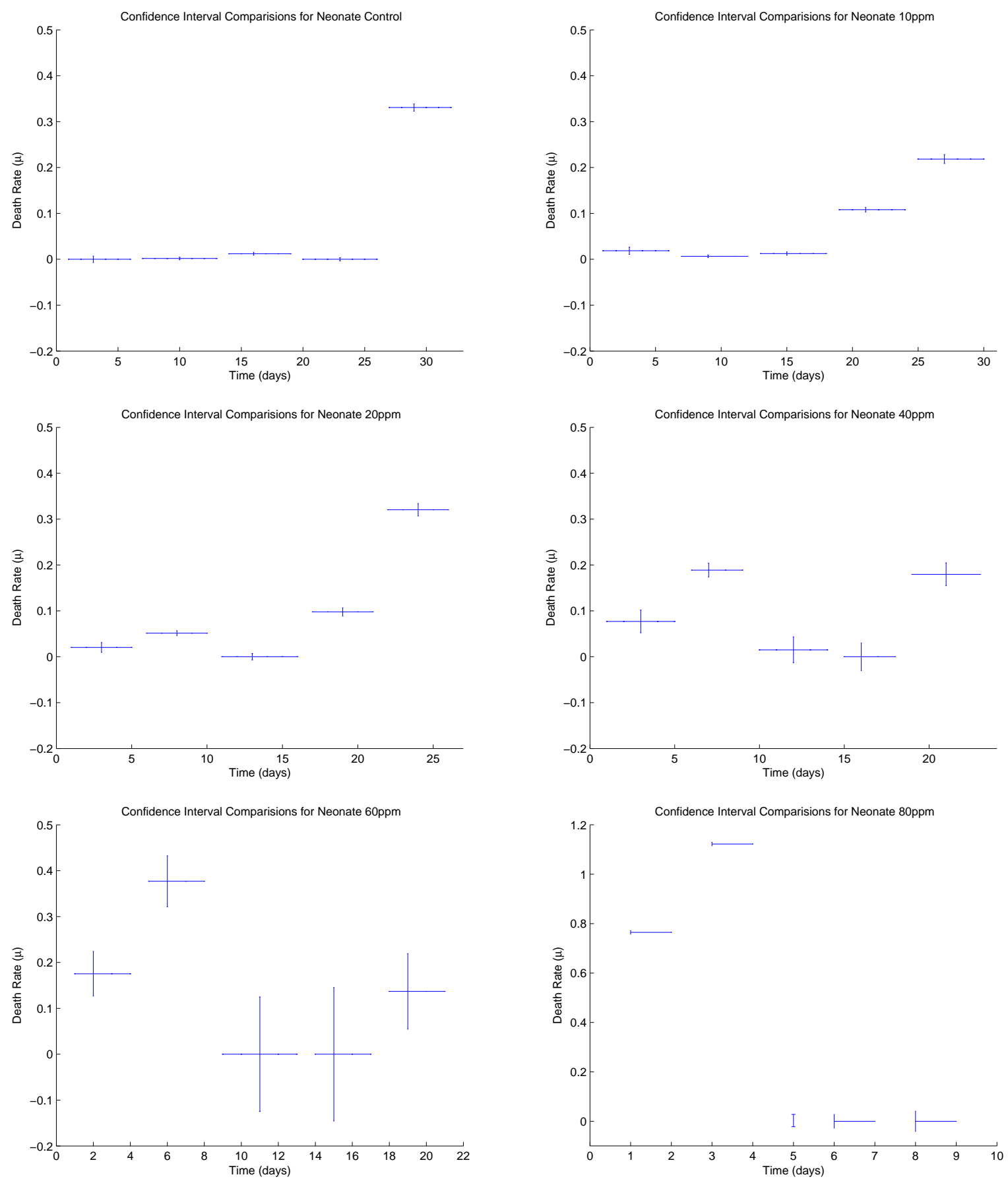

Figure 47: Confidence intervals on $\mu$ for $\mathrm{M}=5$ neonate exposure 

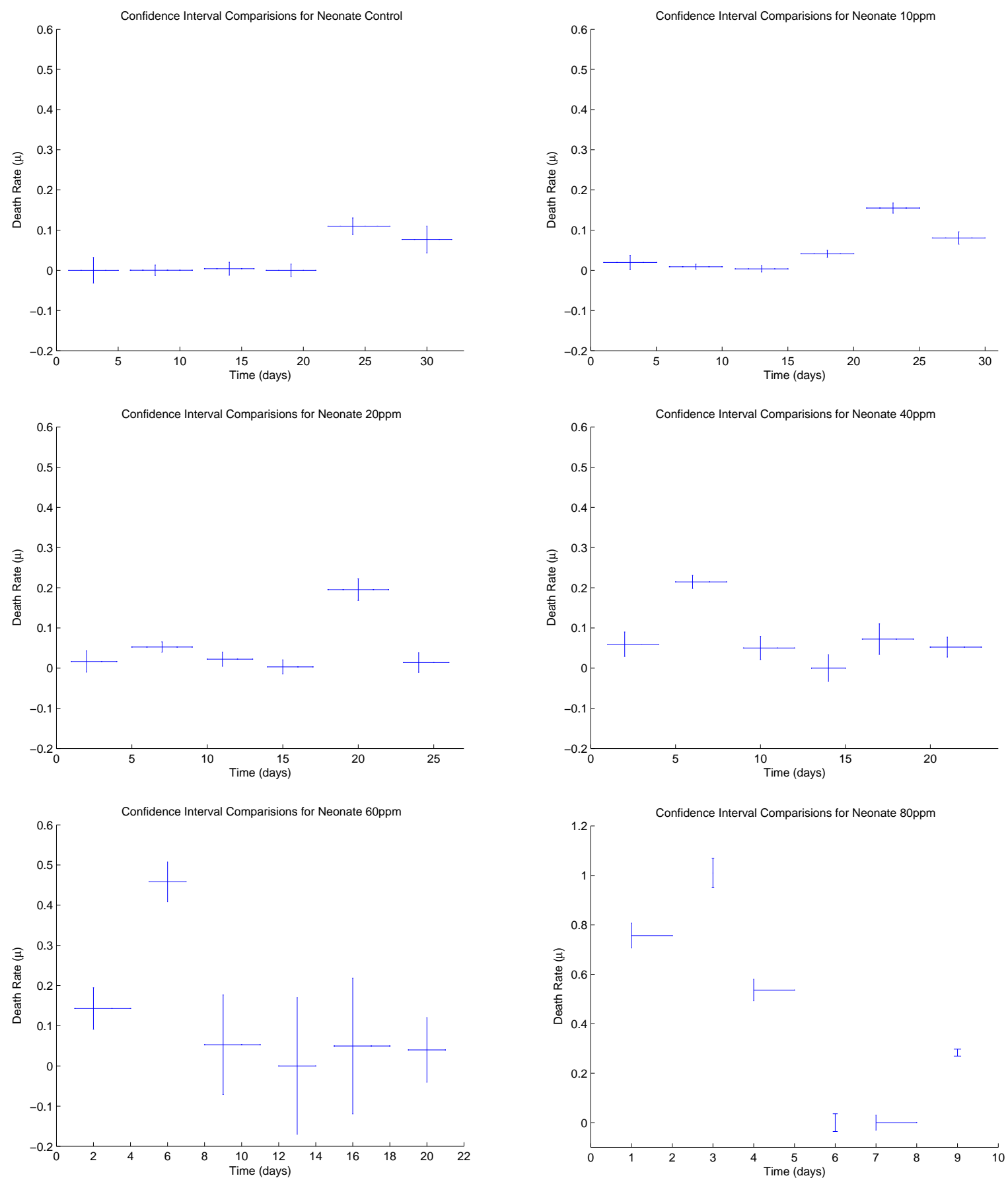

Figure 48: Confidence intervals on $\mu$ for $\mathrm{M}=6$ neonate exposure 
Table 14: Confidence intervals for adult exposure: $\overline{a_{k}} \pm t_{1-\frac{\alpha}{2}} S E\left(a_{k}\right)$

\begin{tabular}{|c|c|c|c|}
\hline & Control & 10ppm & 20ppm \\
\hline \hline $\mathbf{M = 1}$ & $.0173 \pm .0179$ & $.0251 \pm .0228$ & $.0337 \pm .0428$ \\
\hline $\mathbf{M = 2}$ & $.0027 \pm .0061$ & $.0082 \pm .0058$ & $.0134 \pm .0049$ \\
& $.0599 \pm .0037$ & $.0802 \pm .0041$ & $.1118 \pm .0039$ \\
\hline $\mathbf{M = 3}$ & $.0013 \pm .0085$ & $.0081 \pm .0063$ & $.0031 \pm .0060$ \\
& $.0175 \pm .0035$ & $.0215 \pm .0027$ & $.0667 \pm .0027$ \\
& $.1004 \pm .0073$ & $.1505 \pm .0070$ & $.1211 \pm .0074$ \\
\hline $\mathbf{M = 4}$ & $0 \pm .0102$ & $.0019 \pm .0075$ & $0 \pm .0074$ \\
& $.0194 \pm .0039$ & $.0291 \pm .0030$ & $.0437 \pm .0036$ \\
& $.0106 \pm .0052$ & $.0250 \pm .0043$ & $.0752 \pm .0061$ \\
& $.1755 \pm .0093$ & $.2448 \pm .0096$ & $.1735 \pm .0122$ \\
\hline $\mathbf{M = 5}$ & $0 \pm .0076$ & $0 \pm .0083$ & $0 \pm .0100$ \\
& $.0033 \pm .0031$ & $.0263 \pm .0037$ & $.0189 \pm .0037$ \\
& $.0382 \pm .0039$ & $.0189 \pm .0046$ & $.0883 \pm .0057$ \\
& $.0004 \pm .0045$ & $.0883 \pm .0060$ & $.0473 \pm .0081$ \\
& $.2826 \pm .0094$ & $.2667 \pm .0161$ & $.2667 \pm .0174$ \\
\hline $\mathbf{M = 6}$ & $0 \pm .0257$ & $0 \pm .0157$ & $0 \pm .0184$ \\
& $0 \pm .0104$ & $.0163 \pm .0067$ & $.0105 \pm .0064$ \\
& $.0314 \pm .0138$ & $.0337 \pm .0091$ & $.0584 \pm .0087$ \\
& $0 \pm .0139$ & $0 \pm .0094$ & $.0741 \pm .0116$ \\
& $.1059 \pm .0188$ & $.1597 \pm .0144$ & $.1180 \pm .0181$ \\
& $.0573 \pm .0292$ & $.7275 \pm .0672$ & $.2575 \pm .0454$ \\
\hline
\end{tabular}

\begin{tabular}{|l|c|c|c|}
\hline & 40ppm & 60ppm & 80ppm \\
\hline \hline $\mathbf{M = 1}$ & $.0420 \pm .0415$ & $.0417 \pm .0417$ & $.0463 \pm .0477$ \\
\hline $\mathbf{M = 2}$ & $.0172 \pm .0069$ & $.0159 \pm .0072$ & $.0129 \pm .0074$ \\
& $.1651 \pm .0064$ & $.1600 \pm .0066$ & $.1795 \pm .0072$ \\
\hline $\mathbf{M = 3}$ & $.0009 \pm .0053$ & $0 \pm .0066$ & $.0024 \pm .0065$ \\
& $.0999 \pm .0031$ & $.1035 \pm .0037$ & $.1035 \pm .0038$ \\
& $.1760 \pm .0096$ & $.1256 \pm .0090$ & $.1819 \pm .0102$ \\
\hline $\mathbf{M = 4}$ & $0 \pm .0096$ & $0 \pm .0128$ & $.7895 \pm .0106$ \\
& $.0579 \pm .0048$ & $.0520 \pm .0055$ & $0 \pm .0048$ \\
& $.1178 \pm .0094$ & $.1313 \pm .0110$ & $.0649 \pm .0097$ \\
& $.2422 \pm .0262$ & $.1472 \pm .0238$ & $.1249 \pm .0239$ \\
\hline $\mathbf{M = 5}$ & $0 \pm .0110$ & $0 \pm .0083$ & $0 \pm .0142$ \\
& $.0194 \pm .0041$ & $.0108 \pm .0035$ & $.0310 \pm .0063$ \\
& $.1303 \pm .0069$ & $.1529 \pm .0056$ & $.1260 \pm .0100$ \\
& $.0917 \pm .0131$ & $.0441 \pm .0097$ & $.0909 \pm .0185$ \\
& $.3451 \pm .0303$ & $.2919 \pm .0187$ & $.4438 \pm .0409$ \\
\hline $\mathbf{M = 6}$ & $0 \pm .0203$ & $0 \pm .0164$ & $0 \pm .0172$ \\
& $.0012 \pm .0069$ & $0 \pm .0067$ & $.0066 \pm .0072$ \\
& $.1058 \pm .0103$ & $.0919 \pm .0089$ & $.1085 \pm .0098$ \\
& $.0870 \pm .0160$ & $.1305 \pm .0148$ & $.1035 \pm .0162$ \\
& $.1840 \pm .0297$ & $.1121 \pm .0236$ & $.1851 \pm .0280$ \\
& $.0124 \pm .0509$ & $.3201 \pm .0380$ & $.2563 \pm .0426$ \\
\hline
\end{tabular}



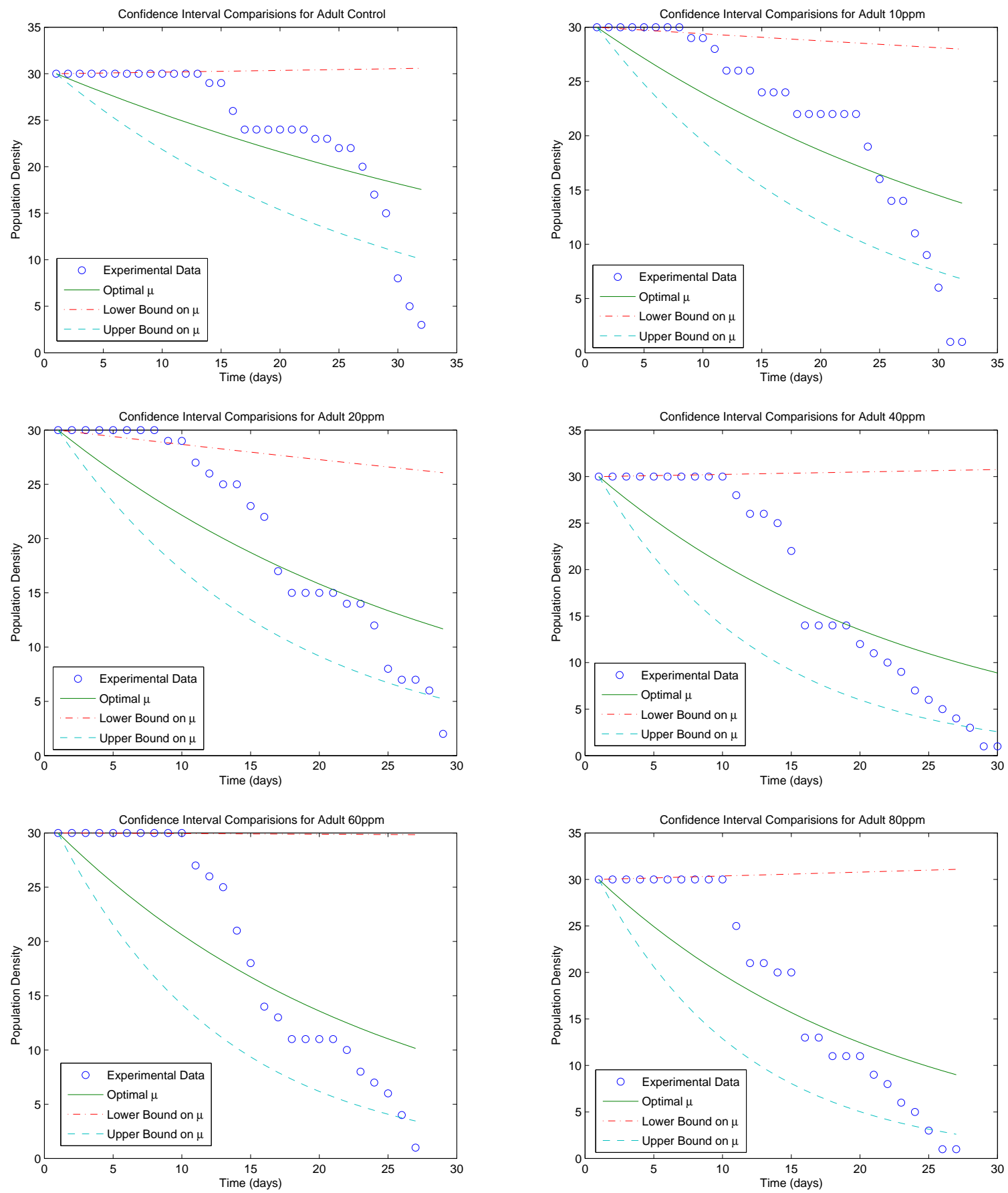

Figure 49: Population densities for adult exposure run with upper and lower bounds of confidence intervals $(\mathrm{M}=1)$ 

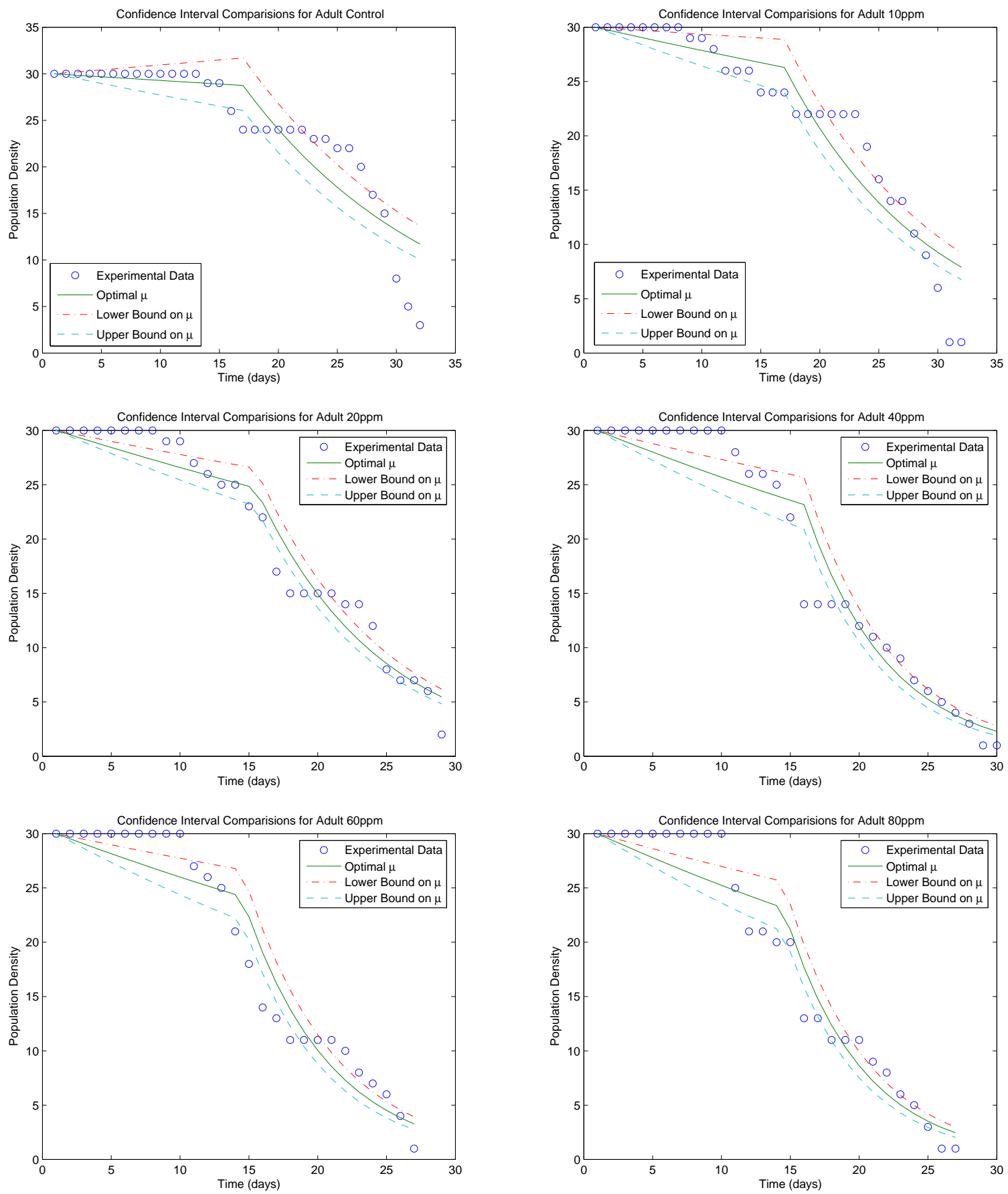

Figure 50: Population densities for adult exposure run with upper and lower bounds of confidence intervals $(\mathrm{M}=2)$ 

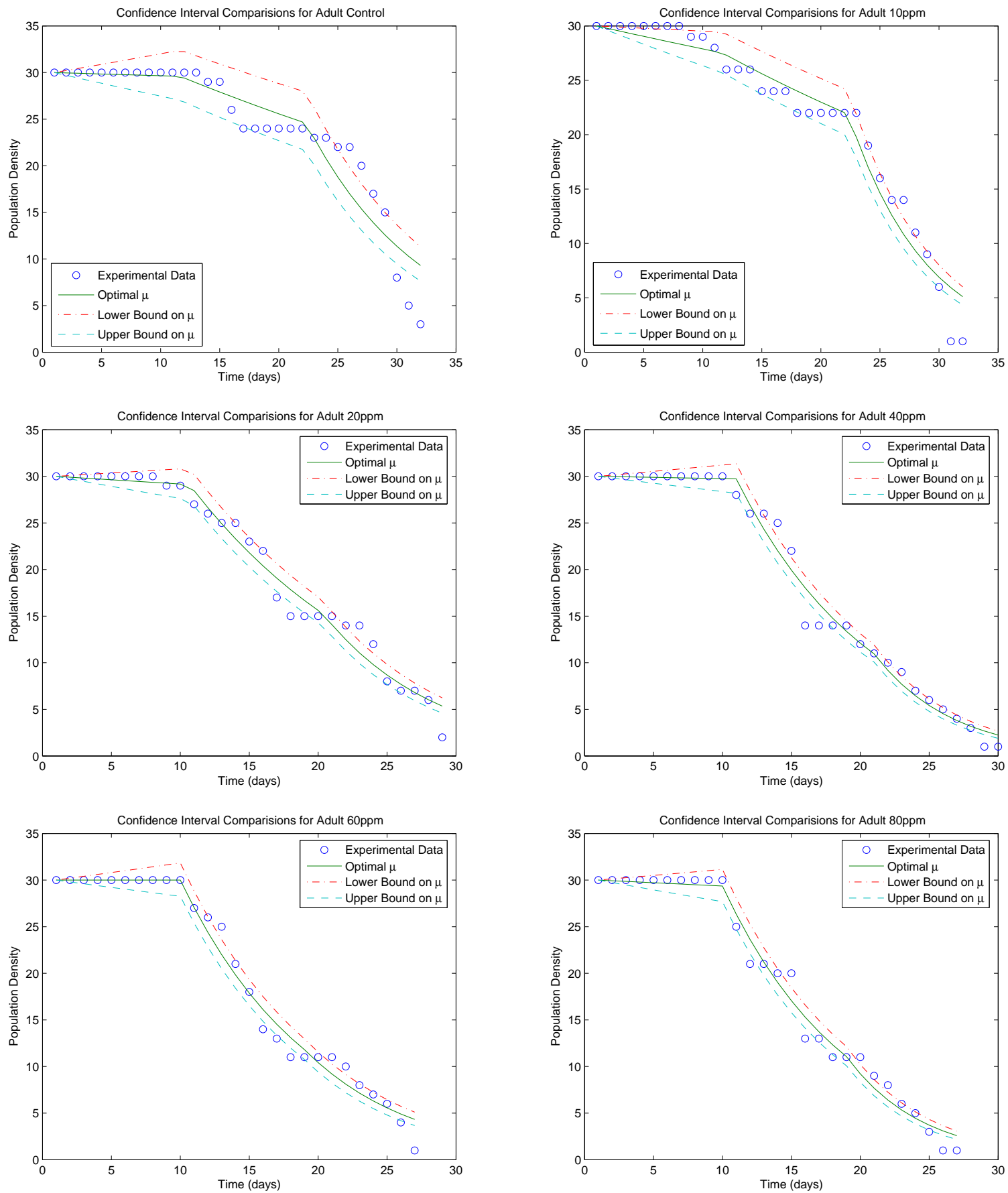

Figure 51: Population densities for adult exposure run with upper and lower bounds of confidence intervals $(\mathrm{M}=3)$ 

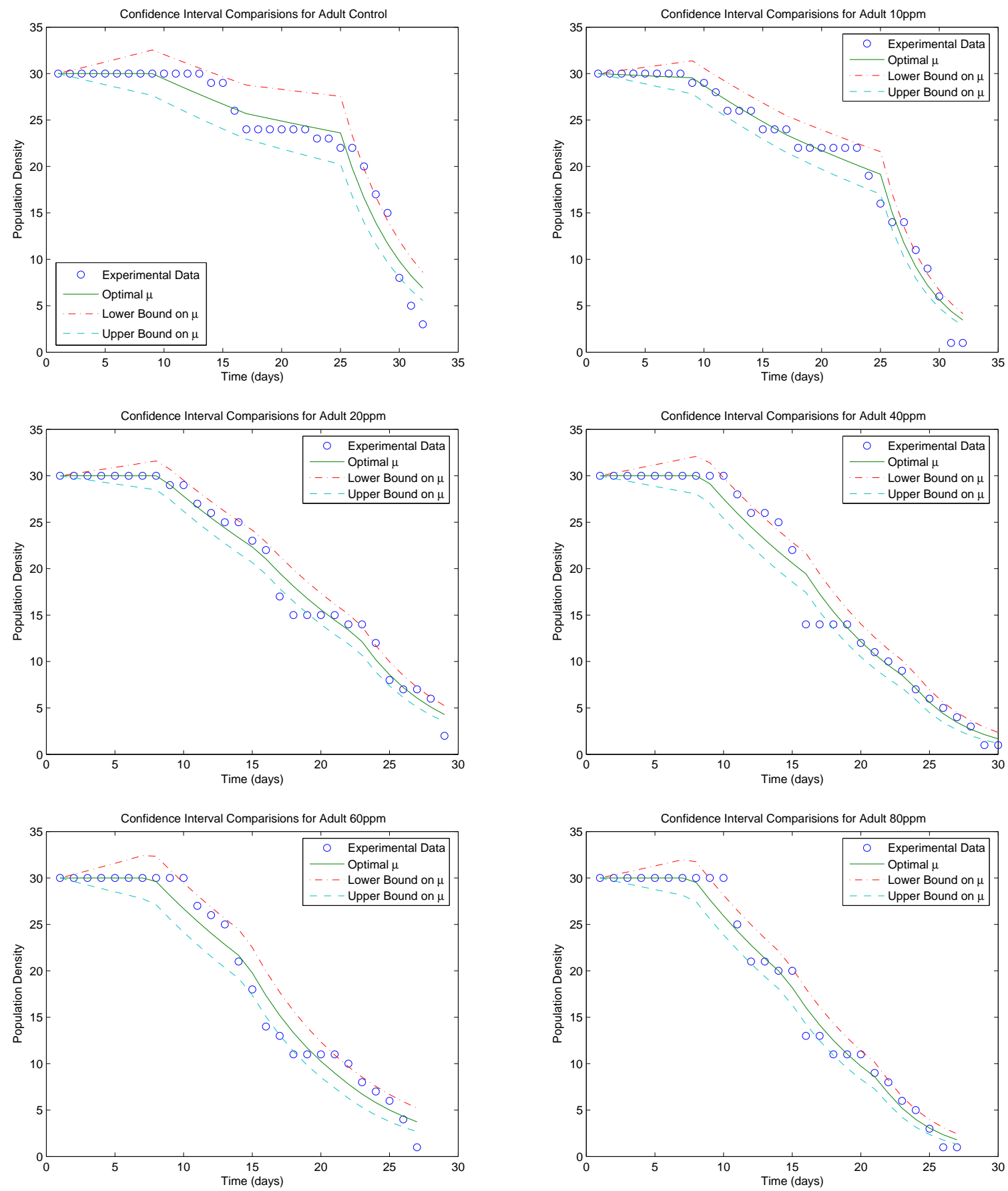

Figure 52: Population densities for adult exposure run with upper and lower bounds of confidence intervals $(\mathrm{M}=4)$ 

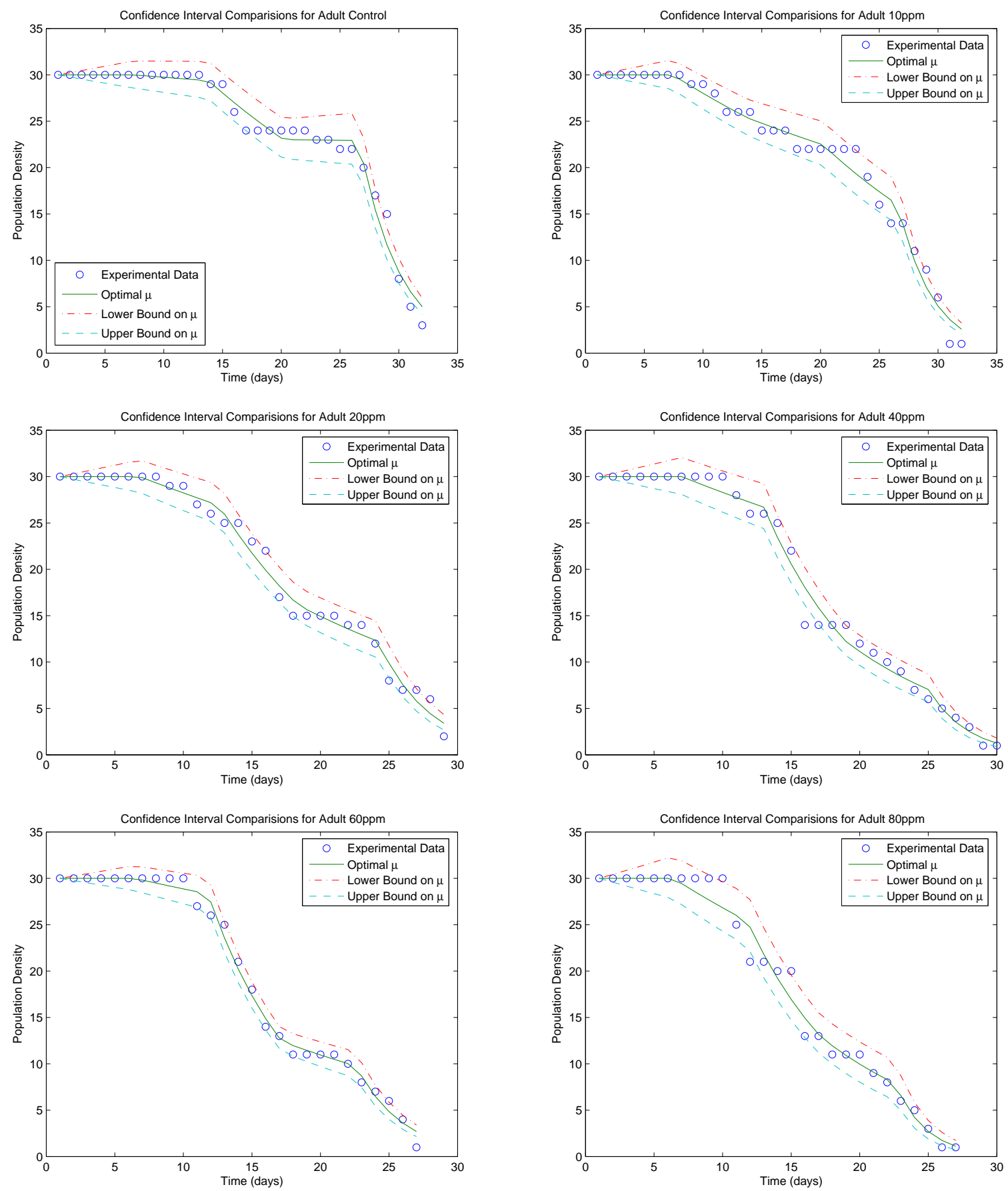

Figure 53: Population densities for adult exposure run with upper and lower bounds of confidence intervals $(\mathrm{M}=5)$ 

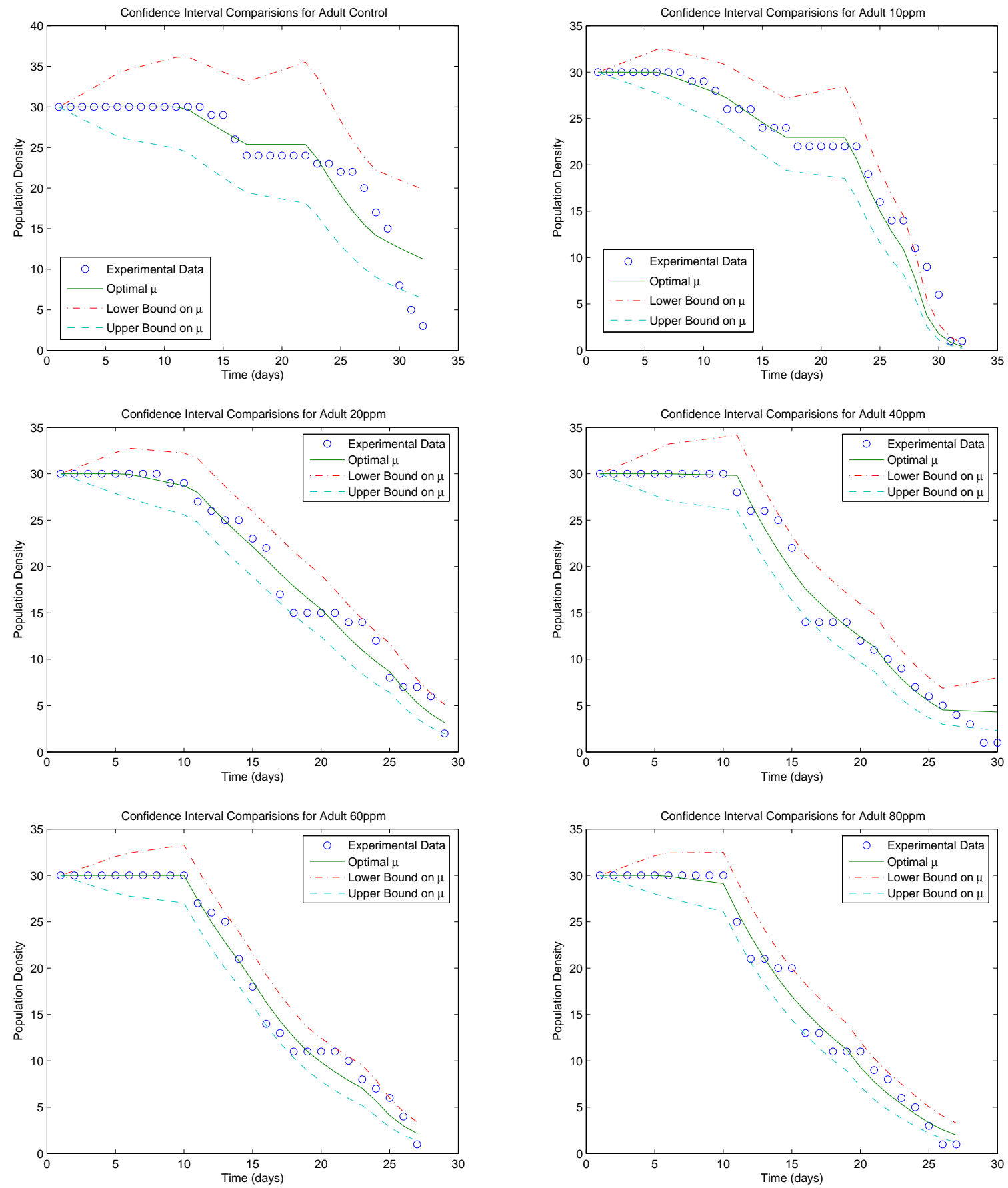

Figure 54: Population densities for adult exposure run with upper and lower bounds of confidence intervals $(\mathrm{M}=6)$ 

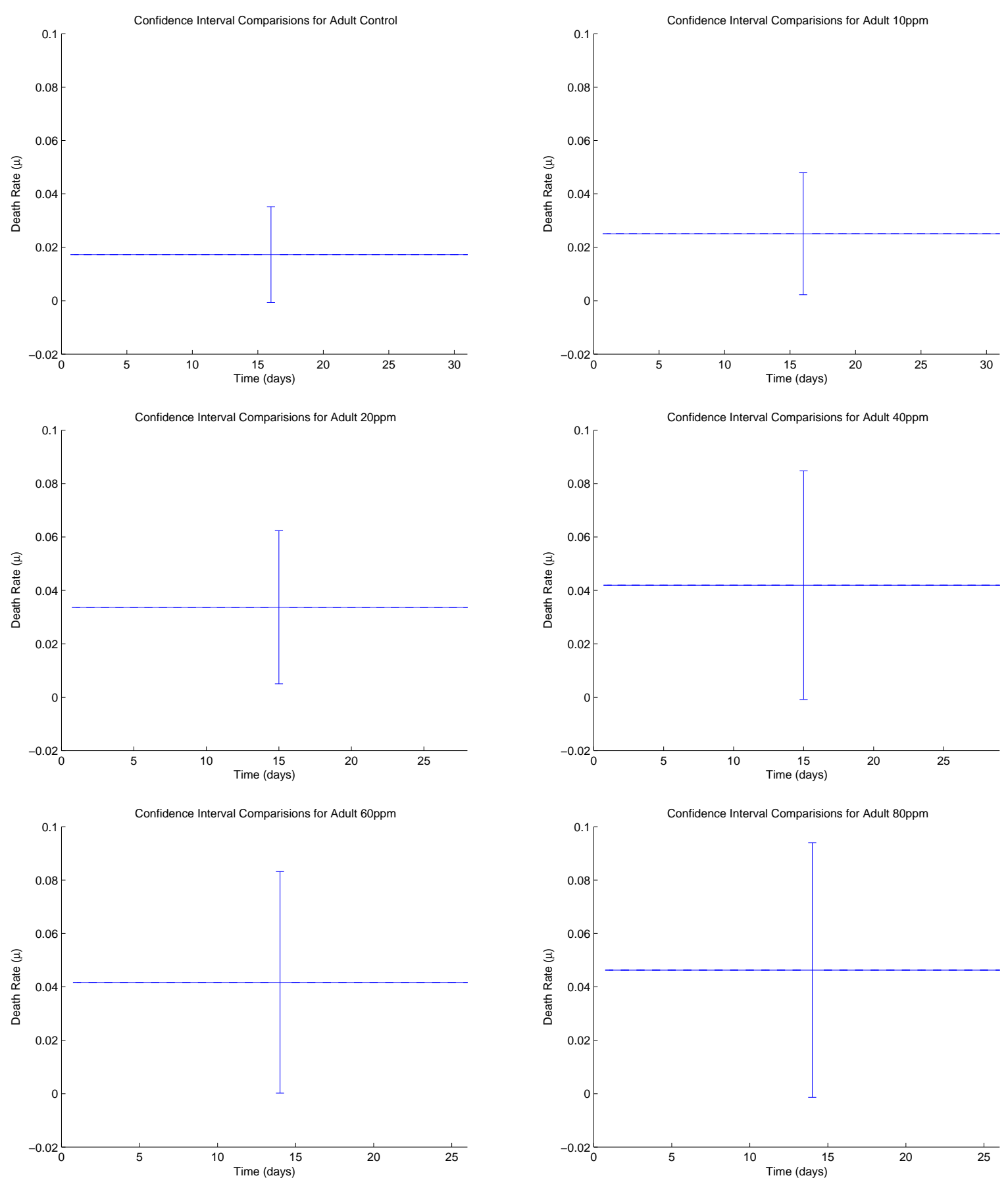

Figure 55: Confidence intervals on $\mu$ for $M=1$ adult exposure 

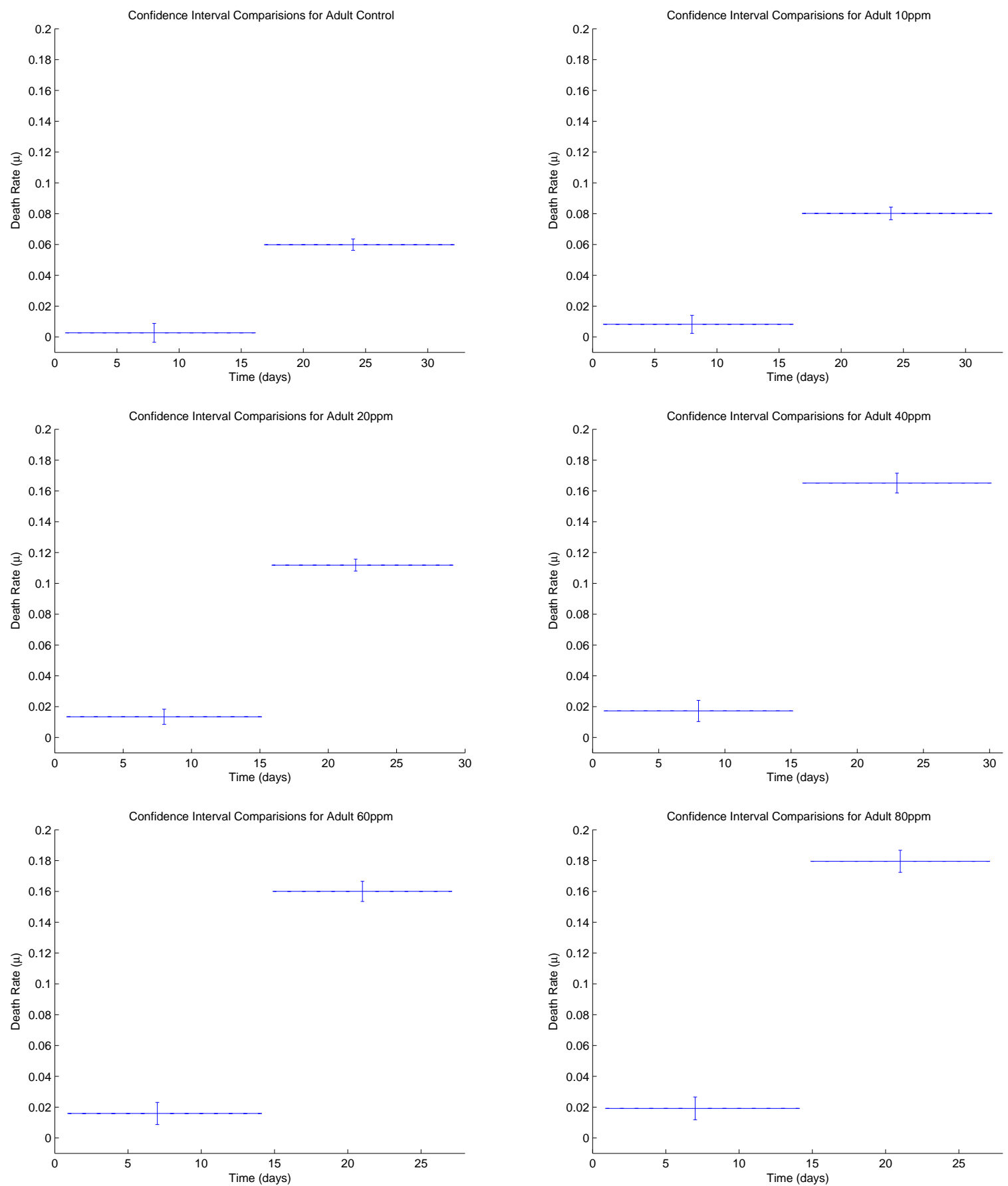

Figure 56: Confidence intervals on $\mu$ for $\mathrm{M}=2$ adult exposure 

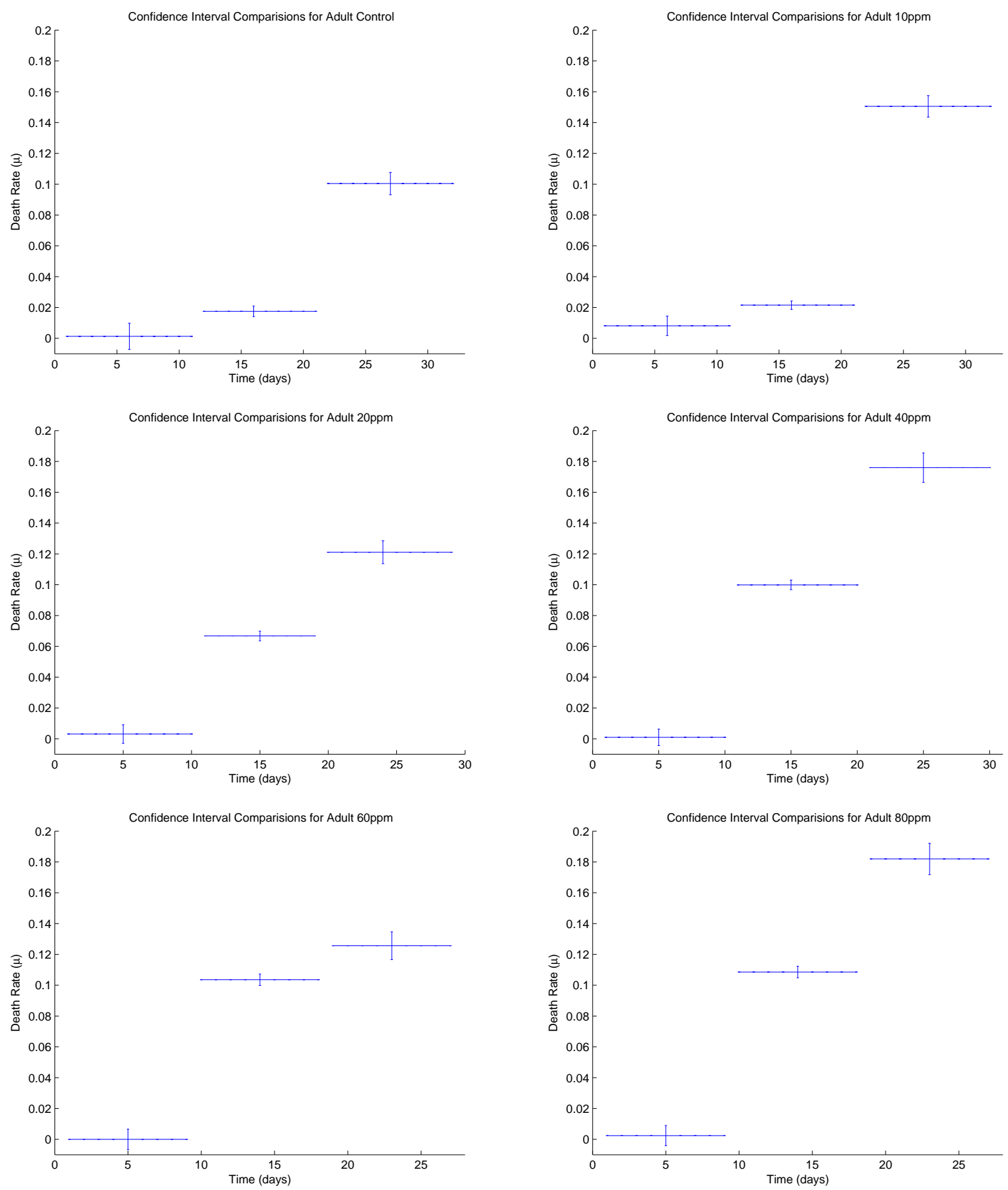

Figure 57: Confidence intervals on $\mu$ for $\mathrm{M}=3$ adult exposure 

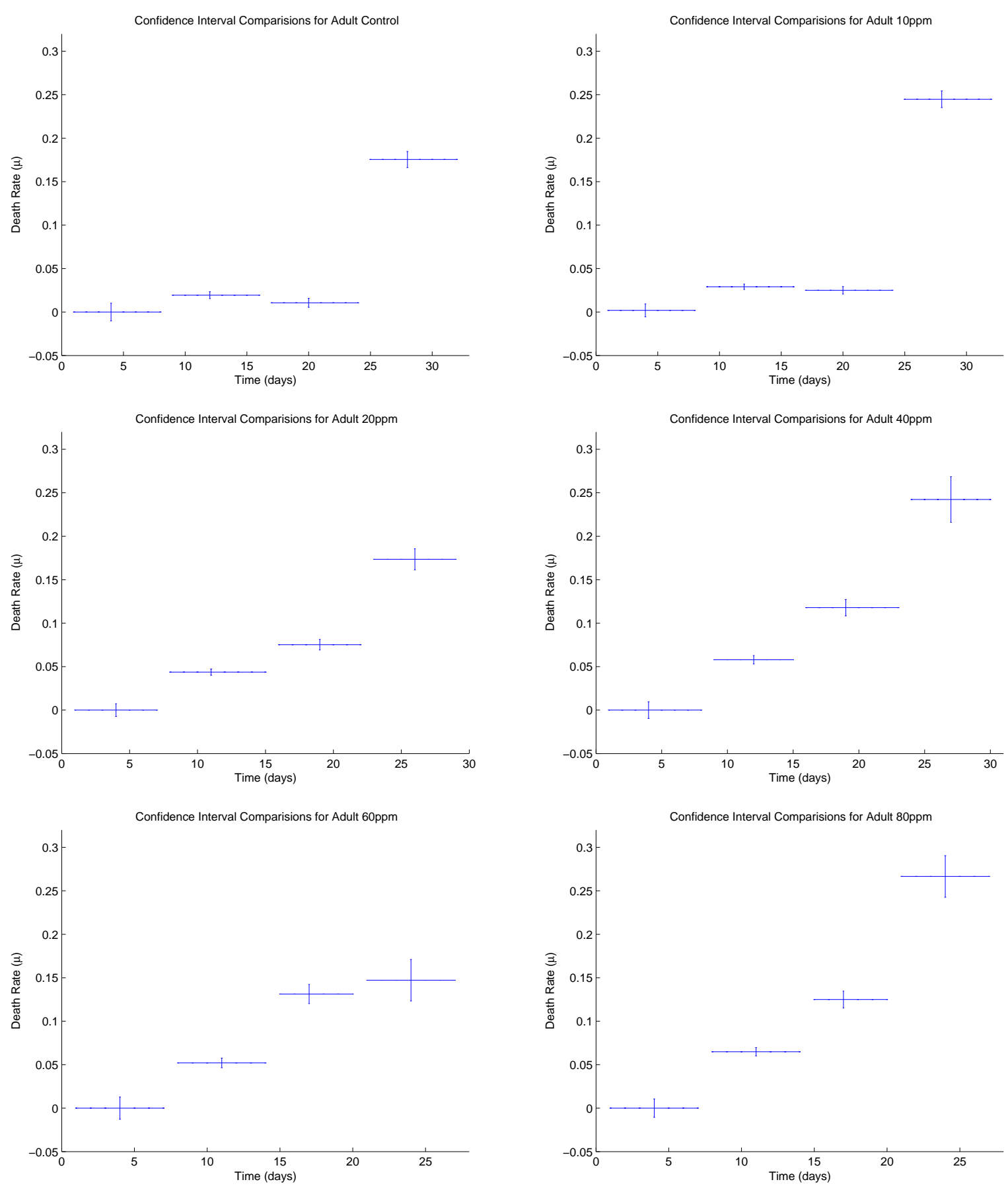

Figure 58: Confidence intervals on $\mu$ for $\mathrm{M}=4$ adult exposure 

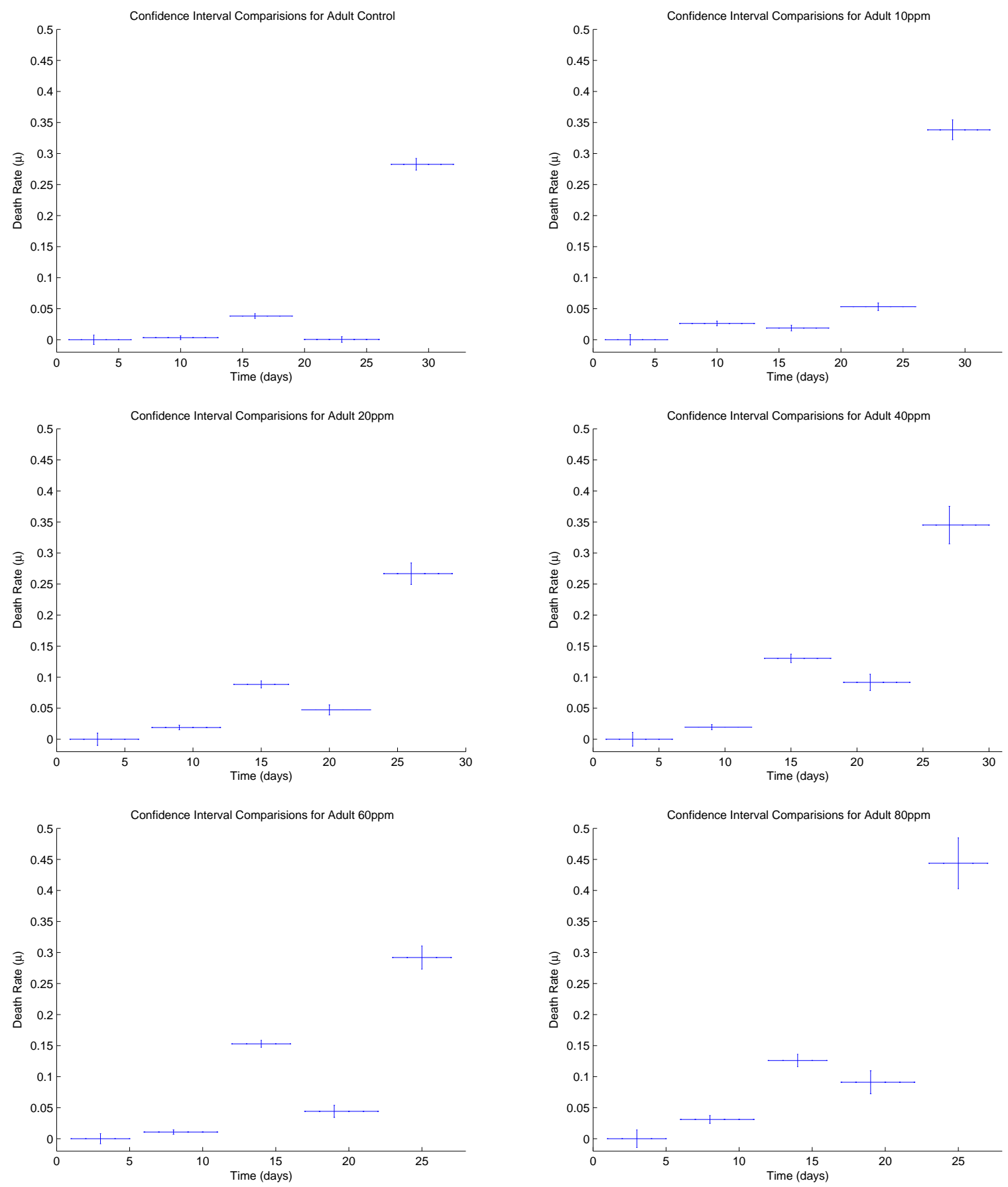

Figure 59: Confidence intervals on $\mu$ for $\mathrm{M}=5$ adult exposure 

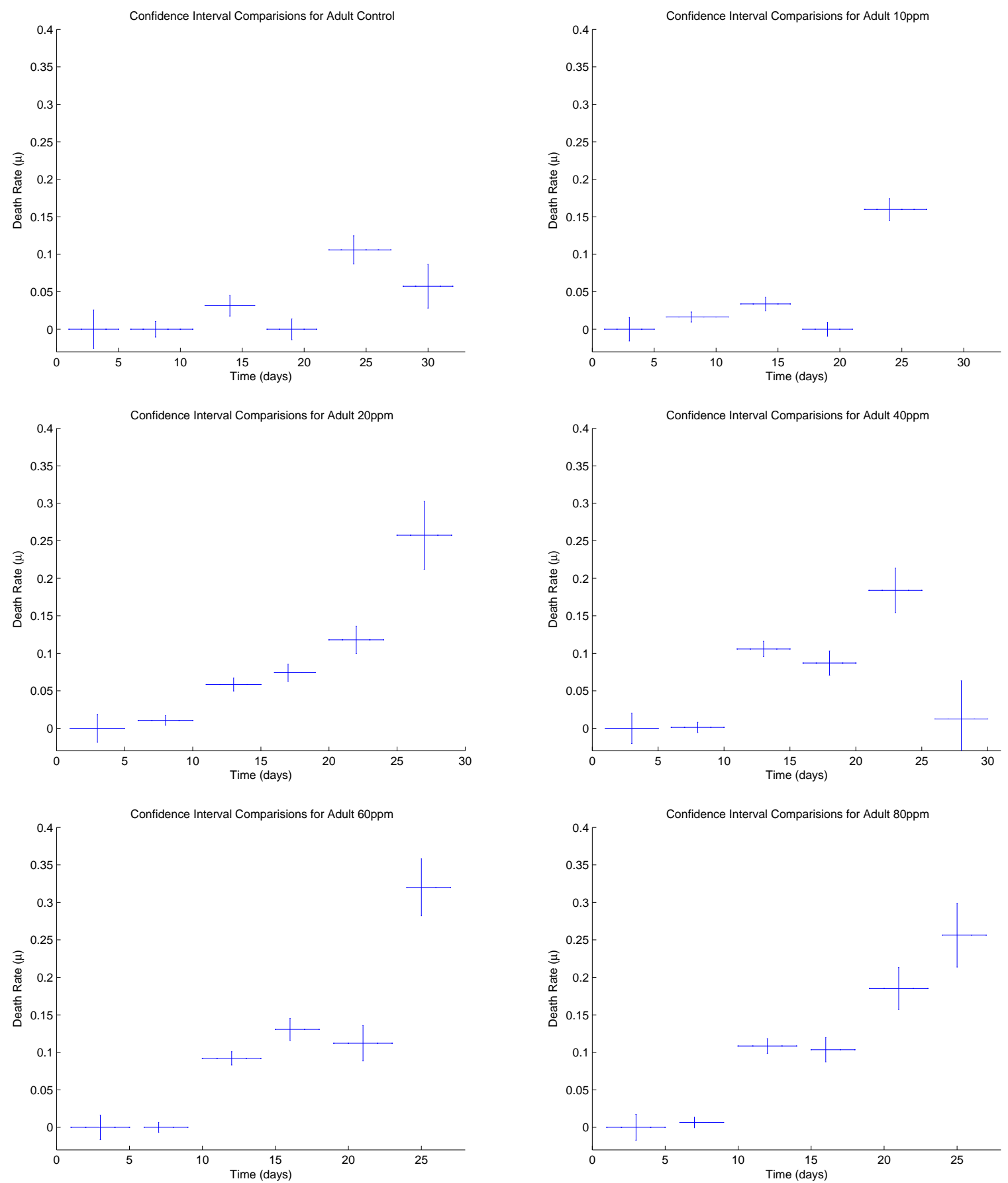

Figure 60: Confidence intervals on $\mu$ for $\mathrm{M}=6$ adult exposure 


\section{Statistical Considerations for Model Comparisons}

The previous section contained our empirical conclusions about the consideration of death rate as a time dependent parameter. Examination of our figures seem to indicate that $\mu^{M}(t)$ does a better job representing our experimental data than does constant $\mu$. However, we would like to be able to actually determine how successful we have been in modeling our data with the time dependent $\mu^{M}(t)$ versus our optimal constant $\mu$. We turn to statistics to help answer this question.

\subsection{Test Statistics}

We use a statistical method known as hypothesis testing to assist us in better understanding our results. This method is detailed in $[\mathrm{BK}]$. We expect that increasing the degrees of freedom of our death rate through parameter refinements will provide us with a reduced cost function value (i.e., a reduced residual sum of squares in the minimized OLS). We wish to ascertain if this reduced cost signifies a truly better fit, statistically speaking, to our experimental data, or if the reduced cost is simply a reflection of reduction due to increased degrees of freedom. Thus we test our null hypothesis $H_{0}$ : additional nodes of $\mu^{M}(t)$ provide us with a significantly better fit to our experimental data, verses our alternative hypothesis $H_{A}$ : additional nodes of $\mu^{M}(t)$ do not provide us with a significantly better fit to our data. We will be using the $\chi^{2}$ statistical test. We compute the following test statistic:

$$
U_{n}=\frac{n\left(J\left(q_{M_{1}}^{*}\right)-J\left(q_{M_{2}}^{*}\right)\right)}{J\left(q_{M_{2}}^{*}\right)},
$$

where $n$ is the number of data points, and $M_{1}$ and $M_{2}$ are the number of nodal points from our optimal $\mu^{M}(t) ; M_{2}>M_{1}$. Again we can only perform statistical calculations on "nested" nodes, those that are refinements of each other. It can be shown, see [BK], that $U_{n}$ is asymptotically (as $n \rightarrow \infty)$ distributed as a $\chi^{2}(s)$ distribution with $s=M_{2}-M_{1}$ degrees of freedom.

The test statistic values one obtains are then compared to values from $\chi^{2}$ Tables (see Table 15). One determines their degrees of freedom and then looks up their test statistic value in the chart to determine their significance level $\alpha$. For example, if a simulation of one node versus three nodes produces a test statistic of 6.5 , then we look under the two degrees of freedom column and determine that our significance level is $\alpha=.05$. This means that we are $95 \%$ confident that the additional two parameters for $\mu^{M}(t)$ provide a better fit to the experimental data than using a constant $\mu$. If on the other-hand, our simulations produced a test statistic of 3.9, we would have to reject our null hypothesis in favor of the alternative hypothesis to determine that the additional parameters were not significant in the cost criterion reduction. 
Table 15: $C h i^{2}(x)$ F-Tables for significance levels $(\alpha)$ and degrees of freedom, $x$

\begin{tabular}{c|c|c|c|c|c} 
& $\chi^{2}(1)$ & $\chi^{2}(2)$ & $\chi^{2}(3)$ & $\chi^{2}(4)$ & $\chi^{2}(5)$ \\
\hline$\alpha=.100$ & 2.706 & 4.604 & 6.251 & 7.779 & 9.236 \\
\hline$\alpha=.050$ & 3.841 & 5.991 & 7.815 & 9.488 & 11.071 \\
\hline$\alpha=.025$ & 5.024 & 7.378 & 9.348 & 11.143 & 12.833 \\
\hline$\alpha=.010$ & 6.635 & 9.210 & 11.345 & 13.277 & 15.086 \\
\hline$\alpha=.005$ & 7.879 & 10.597 & 12.838 & 14.860 & 16.750 \\
\hline$\alpha=.001$ & 10.828 & 13.816 & 16.266 & 18.467 & 20.515 \\
\hline
\end{tabular}

\subsection{Results}

Our calculated $\chi^{2}$ test statistics for adult exposure can be found in Table 16. Most of our test statistics are greater than those values located in the $\chi^{2}$ chart. With the exception of three versus six nodes, we are able to accept our null hypothesis with a significance level of $\alpha=.001$. Thus we are $99.9 \%$ confident that each additional nodal value in $\mu^{M}(t)$ provides us with a better estimate of our experimental data. For 10ppm with three versus six nodes, we are $99 \%$ confident that the additional three nodes in $\mu^{M}(t)$ provide us with a better estimate of the experimental data. However, for the rest of the exposure levels we reject our null hypothesis and determine that the additional three nodes in $\mu^{M}(t)$ are not significant.

Table 16: $C h i^{2}(x)$ test statistics and corresponding significance levels $(\alpha)$ for adult exposure

\begin{tabular}{|c|c|c|c|c|c|c|}
\hline Nodes & Control & 10ppm & 20ppm & 40ppm & 60ppm & 80ppm \\
\hline 6 vs. 1 & 118.3 & 330.8 & 291.5 & 532.1 & 452.1 & 464.4 \\
& $\alpha=.001$ & $\alpha=.001$ & $\alpha=.001$ & $\alpha=.001$ & $\alpha=.001$ & $\alpha=.001$ \\
\hline 5 vs. 1 & 704.7 & 584.1 & 582.1 & 674.7 & $1,125.6$ & 378.9 \\
& $\alpha=.001$ & $\alpha=.001$ & $\alpha=.001$ & $\alpha=.001$ & $\alpha=.001$ & $\alpha=.001$ \\
\hline 4 vs. 1 & 224.5937 & 451.0236 & 389.6690 & 318.4850 & 247.0356 & 390.7992 \\
& $\alpha=.001$ & $\alpha=.001$ & $\alpha=.001$ & $\alpha=.001$ & $\alpha=.001$ & $\alpha=.001$ \\
\hline 3 vs. 1 & 100.3179 & 234.2025 & 279.3405 & 518.8923 & 410.7597 & 453.7123 \\
& $\alpha=.001$ & $\alpha=.001$ & $\alpha=.001$ & $\alpha=.001$ & $\alpha=.001$ & $\alpha=.001$ \\
\hline 2 vs. 1 & 47.8803 & 68.2875 & 132.4636 & 96.8980 & 94.8659 & 100.4630 \\
& $\alpha=.001$ & $\alpha=.001$ & $\alpha=.001$ & $\alpha=.001$ & $\alpha=.001$ & $\alpha=.001$ \\
\hline 6 vs. 3 & 4.34 & 11.61 & 1.14 & .721 & 2.547 & .599 \\
& Reject & $\alpha=.010$ & Reject & Reject & Reject & Reject \\
\hline 4 vs. 2 & 70.7914 & 122.124 & 46.196 & 52.3855 & 33.7139 & 61.5008 \\
& $\alpha=.001$ & $\alpha=.001$ & $\alpha=.001$ & $\alpha=.001$ & $\alpha=.001$ & $\alpha=.001$ \\
\hline
\end{tabular}

The calculated $\chi^{2}$ test statistics for neonate exposure can be found in Table 17 . We see that most of our test statistics correspond to a high significance level. However, as exposure levels 
increase we obtain lower significance levels and are sometimes forced to reject our null hypothesis and determine that additional parameters for $\mu^{M}(t)$ are not significant for some exposure levels and nodal points.

Table 17: $C h i^{2}(x)$ test statistics and corresponding significance levels $(\alpha)$ for neonate exposure

\begin{tabular}{|l|c|c|c|c|c|c|}
\hline Nodes & Control & 10ppm & 20ppm & 40ppm & 60ppm & 80ppm \\
\hline 6 vs. 1 & 91.5 & 581.1 & 264.4 & 60.8 & 26.4 & 235.6 \\
& $\alpha=.001$ & $\alpha=.001$ & $\alpha=.001$ & $\alpha=.001$ & $\alpha=.01$ & $\alpha=.001$ \\
\hline 5 vs. 1 & $1,268.0$ & 976.2 & 308.9 & 41.9 & 13.7 & 81.4 \\
& $\alpha=.001$ & $\alpha=.001$ & $\alpha=.001$ & $\alpha=.001$ & $\alpha=.01$ & $\alpha=.001$ \\
\hline $\mathbf{4}$ vs. 1 & 244.4486 & 499.7015 & 179.8627 & 16.7996 & 6.5043 & 23.2973 \\
& $\alpha=.001$ & $\alpha=.001$ & $\alpha=.001$ & $\alpha=.001$ & $\alpha=.1$ & $\alpha=.001$ \\
\hline 3 vs. 1 & 90.9588 & 399.1722 & 179.0555 & 7.3400 & 3.0641 & 9.6788 \\
& $\alpha=.001$ & $\alpha=.001$ & $\alpha=.001$ & $\alpha=.05$ & Reject & $\alpha=.025$ \\
\hline 2 vs. 1 & 31.6944 & 120.7690 & 32.9080 & 9.8128 & 4.8108 & 21.6565 \\
& $\alpha=.001$ & $\alpha=.001$ & $\alpha=.001$ & $\alpha=.005$ & $\alpha=.05$ & $\alpha=.001$ \\
\hline 6 vs. 3 & .136 & 12.67 & 10.79 & 40.50 & 20.34 & 108.9 \\
& Reject & $\alpha=.10$ & $\alpha=.005$ & $\alpha=.001$ & $\alpha=.001$ & $\alpha=.001$ \\
\hline $\mathbf{4}$ vs. 2 & 106.887 & 75.3998 & 64.8609 & 4.8973 & 1.3779 & .4821 \\
& $\alpha=.001$ & $\alpha=.001$ & $\alpha=.001$ & $\alpha=.1$ & Reject & Reject \\
\hline
\end{tabular}

Although we reject an additional nodal values versus a constant death rate, some exposure levels for two versus four nodal values and some exposure levels for three versus six nodes, we are able to determine that additional nodal values in $\mu^{M}(t)$ provide us with a better fit to the experimental data except for the high exposure levels with immediate deaths in the population due to the pesticide sprays.

The fact that we obtain significance at a high confidence level for both population classes and for most exposure levels leads us to conclude that death rate should be considered as a time dependent parameter. We have shown that the method used to determine the constant death rate using the Leslie simulations does not produce values that make sense when compared with experimental data. We also argue that Leslie does not produce the type of results needed to understand sublethal effects.

\section{Concluding Remarks}

For the Sinko Streifer model we developed earlier, we used a linear interpolation of results from Leslie to determine the recruitment rate $R(t)$. In theory, one might rather use the fecundity values we know from our data. This would involve solving the integral from equation (5), which cannot be calculated in a traditional manner. We would then treat recruitment in a different way. We 
hope to write the PDE in "weak" or "variational form", in order to solve the now coupled system. If we were successful, we will have an altered SS, which may produce different results.

We would also like to use the model for different species and scenarios to test its applicability for use of ecologists.

\section{Acknowledgments}

This work was supported in part (HTB and LKD) by the US Air Force Office of Scientific Research under grant AFOSR FA9550-01-1-0220, and in part (LKD) by a CRSC/Lord Corporation Fellowship.

\section{References}

[B] L. C. Birch, The intrinsic rate of natural increase of an insect population, Journal of Animal Ecology, 17: 15-26, 1948.

[BK] H. T. Banks and K. Kunsich, Estimation Techniques for Distributed Parameter Systems, Birkhauser, Boston, 1989.

[BT] H. T. Banks and H. T. Tran, Mathematical and Experimental Modeling of Physical and Biological Processes, SIAM Publications, Philadelphia, to appear 2005.

[C] J. R. Carey, Applied Demography for Biologists with Special Emphasis on Insects, Oxford University Press, Oxford, England, 1993.

[Ca] H. Caswell, Matrix Population Models, Sinauer Associates, Inc. Publishers, Sunderland, Massachusetts, 2001.

[CL] J. R. Carey and P. Liedo, Mortality Dynamics of Insects: General principals derived from aging research on the mediterranean fruit fly (diptera: Tephritidae), American Entomologist, 45(1): 49-55, 1999.

[D] L. K. Dick, Prediction of life history traits in invertebrate species exposed to pesticide, Master Thesis, North Carolina State University, 2005.

[H] S. M Henson, Leslie matrix models as "stroboscopic snapshots" of McKendrick PDE models, Journal of Mathematical Biology, 37: 309-328, 1998.

[K] Kot, Mark, Elements of Mathematical Ecology, Cambridge University Press, Cambridge, 2001.

[Ke] C. T. Kelley, Iterative Methods for Optimization, Society for Industrial and Applied Mathematics, Philadelphia, 1999. 
[L] P. H. Leslie, On the use of matrices in certain population mathematics, Biometrika, 33: 183$212,1945$.

[LW] J. Ladin and U. Wennergren, Population growth and structure in a variable environment, Oecologia, 93: 394-405, 1993.

[M] A. G. McKendrick, Applications of mathematics sto medical problems, Proceedings of the Edinburgh Mathematical Society, 40: 98-130, 1926.

[OW] M. D. Ohman, S. N. Wood, Mortality estimation for plankonic copepods: Pseudocalanus newmani in a temperate fjord, Limnology and Oceeanogrpahy, 41: 126-135, 1996.

[RS] T. M. Rangus and J. D. Stark, Lethal and sublethal effects of the neem insecticide formulation, 'Margosan-O', on the pea aphid, Pesticide Science, 41: 155-160, 1994.

[SS] J. W. Sinko and W. Streifer, A new model for age-size structure of a population, Ecology, 48(6): 910-918, 1967.

[SBV] J. D. Stark, J. E. Banks, and R. Vargas, How risky is risk assessment: The role that life history strategies play in susceptibility of species to stress, PNAS, 101: 732-736, 2004.

[SW] J. D. Stark and U. Wennergren, Can population effects of pesticides be predicted from demographic toxicological studies?, Journal of Economic Entomology, 88:1089-1096, 1995.

[U] G. Uribe, On the relationship between continuous and discrete models for size-structured population dynamics, Doctoral dissertation, University of Arizona, 1993.

[VF] H. Von Foerster, Some remarks on changing populations, Pages 382-407 in F. Stohlman Jr, editors, The Kinetics of Cellular Proliferation, Grune \& Stratton, New York, 1959.

[W] W. K. Walthall and J. D. Stark, Comparison of two population-level ecotoxicological endpoints: The intrinsic $\left(r_{m}\right)$ and instantaneous $\left(r_{i}\right)$ rates of increase, Environmental Toxicology and Chemistry, 16: 1068-1073, 1997.

[Wo] S. N. Wood, Inverse Problems and Structured-Population Dynamics, Pages 555-586 in S. Tuljapurkar and H. Caswell, editors. Structured-Population Models in Marine, Terrestrial, and Freshwater Systems, Chapman and Hall, New York, 1997.

[Wo2] S. N. Wood, Spline models of biological population dynamics: How to estimate mortality rates for stage structured populations with dimorphic life histories, Journal of Mathematics Applied in Medicine $\&$ Biology, 11: 61-78, 1992. 
[Wo3] S. N. Wood, Obtaining birth and mortality patterns from structured population trajectories, Ecological Monographs, 64(1): 23-44, 1994.

[WMS] D. D. Wackerly, W. Mendenhall III, and R. L. Scheaffer, Mathematical Statistics with Applications, Duxbury Thompson Learning, USA, 2002.

[WS] U. Wennergren and J. D. Stark, Modeling long-term effects of pesticides on populations: Beyond just counting dead animals, Ecological Applications, 10: 295-302, 2000. 